\title{
The Chandra Multiwavelength Project: Optical Follow\#up of Serendipitous Chandra Sources
}

\section{Citation}

Green, P. J., J. D. Silverman, R. A. Cameron, D.\#W. Kim, B. J. Wilkes, W. A. Barkhouse, A. LaCluyze, et al. 2004. "The Chandra Multiwavelength Project: Optical Follow\#up of Serendipitous Chandra Sources." The Astrophysical Journal Supplement Series 150 (1) (January): 43-71. doi:10.1086/379818.

\section{Published Version}

doi:10.1086/379818

\section{Permanent link}

http://nrs.harvard.edu/urn-3:HUL.InstRepos:30212197

\section{Terms of Use}

This article was downloaded from Harvard University's DASH repository, and is made available under the terms and conditions applicable to Other Posted Material, as set forth at http:// nrs.harvard.edu/urn-3:HUL.InstRepos:dash.current.terms-of-use\#LAA

\section{Share Your Story}

The Harvard community has made this article openly available.

Please share how this access benefits you. Submit a story.

\section{Accessibility}




\title{
THE CHANDRA MULTIWAVELENGTH PROJECT: OPTICAL FOLLOW-UP OF SERENDIPITOUS CHANDRA SOURCES
}

\author{
P. J. Green, ${ }^{1,2}$ J. D. Silverman, ${ }^{1,2,3}$ R. A. Cameron, ${ }^{1}$ D.-W. Kim, ${ }^{1}$ B. J. Wilkes, ${ }^{1}$ W. A. Barkhouse, ${ }^{1}$ A. LaCluyzé, ${ }^{2,4}$ \\ D. Morris, ${ }^{5}$ A. Mossman, ${ }^{1}$ H. Ghosh, ${ }^{1}$ J. P. Grimes, ${ }^{6}$ B. T. Jannuzi,${ }^{7}$ H. Tananbaum, ${ }^{1}$ T. L. Aldcroft,${ }^{1}$ \\ J. A. Baldwin, ${ }^{4}$ F. H. Chaffee, ${ }^{8}$ A. Dey, ${ }^{7}$ A. Dosaj, ${ }^{2,9}$ N. R. Evans, ${ }^{1}$ X. Fan, ${ }^{10}$ C. Foltz, ${ }^{11}$ T. Gaetz, ${ }^{1}$ \\ E. J. Hooper, ${ }^{12}$ V. L. Kashyap ${ }^{1}$ S. Mathur, ${ }^{13}$ M. B. McGarry, ${ }^{1}$ E. Romero-Colmenero, ${ }^{14}$ \\ M. G. Smith, ${ }^{15}$ P. S. Smith ${ }^{2,16}$ R. C. Smith, ${ }^{15}$ G. Torres, ${ }^{1}$ \\ A. Vikhlinin, ${ }^{1}$ AND D. R. WiK ${ }^{17}$ \\ Received 2003 January 3; accepted 2003 September 5
}

\begin{abstract}
We present follow-up optical $g^{\prime}, r^{\prime}$, and $i^{\prime}$ imaging and spectroscopy of serendipitous X-ray sources detected in six archival Chandra images included in the Chandra Multiwavelength Project (ChaMP). Of the 486 X-ray sources detected between $3 \times 10^{-16}$ and $2 \times 10^{-13}$ (with a median flux of $3 \times 10^{-15}$ ) ergs $\mathrm{cm}^{-2} \mathrm{~s}^{-1}$, we find optical counterparts for 377 (78\%), or $335(68 \%)$ counting only unique counterparts. We present spectroscopic classifications for 125 objects, representing $75 \%$ of sources with $r^{*}<21$ optical counterparts $\left(63 \%\right.$ to $\left.r^{*}=22\right)$. Of all classified objects, $63(50 \%)$ are broad-line active galactic nuclei (AGNs), which tend to be blue in $\left(g^{*}-r^{*}\right)$ colors. X-ray information efficiently segregates these quasars from stars, which otherwise strongly overlap in these SDSS colors until $z>3.5$. We identify 28 sources $(22 \%)$ as galaxies that show narrow emission lines, while $22(18 \%)$ are absorption line galaxies. Eight galaxies lacking broad-line emission have X-ray luminosities that require they host an AGN $\left(\log L_{X}>43\right)$. Half of these have hard X-ray emission suggesting that high gas columns obscure both the X-ray continuum and the broad emission line regions. We find objects in our sample that show signs of X-ray or optical absorption, or both, but with no strong evidence that these properties are coupled. ChaMP's deep X-ray and optical imaging enable multiband selection of small and/or high-redshift groups and clusters. In these six fields we have discovered three new clusters of galaxies, two with $z>0.4$, and one with photometric evidence for a similar redshift.
\end{abstract}

Subject headings: galaxies: active — quasars: general — surveys — X-rays: galaxies

On-line material: color figures

\section{INTRODUCTION}

\subsection{X-Ray Surveys and the Cosmic X-Ray Background}

$X$-ray surveys provide fundamental advances in our knowledge of the X-ray universe and indeed the universe as a whole (e.g., the Einstein Medium Sensitivity Survey (Stocke et al. 1991); the Cambridge-Cambridge ROSAT Serendipitous Survey (Boyle et al. 1997); ROSAT International X-ray/Optical Survey (Page et al. 1996), the $A S C A$ Large Sky Survey (Akiyama et al. 2000). ROSAT (0.1-2.4 keV) and more recently Chandra $(0.3-8 \mathrm{keV})$ have resolved $80 \%-90 \%$ of the

\footnotetext{
${ }^{1}$ Harvard-Smithsonian Center for Astrophysics, 60 Garden Street, Cambridge, MA 02138; pgreen@cfa.harvard.edu.

${ }^{2}$ Visiting Astronomer, Kitt Peak National Observatory and/or Cerro Tololo Inter-American Observatory, National Optical Astronomy Observatories, operated by the Association of Universities for Research in Astronomy, Inc. (AURA), under cooperative agreement with the National Science Foundation.

3 P.O. Box 3818, Astronomy Department, University of Virginia, Charlottesville, VA 22903-3818.

${ }^{4}$ Department of Physics and Astronomy, Michigan State University, East Lansing, MI 48824-1116.

5 Department of Astronomy and Astrophysics, Pennsylvania State University, 525 Davey Laboratory, University Park, PA 16802.

6 Center for Astrophysical Sciences, Johns Hopkins University, Baltimore, MD 21218-2686.

7 National Optical Astronomy Observatory, P.O. Box 26732, Tucson, AZ 85726-6732.

${ }^{8}$ California Association for Research in Astronomy, W. H. Keck Observatory, 65-1120 Mamalahoa Highway, Kamuela, HI 96734.

9 Department of Physics, University of Wisconsin-Madison, 1150 University Avenue, Madison, WI 53706.
}

cosmic X-ray background (CXRB) into discrete sources (Hasinger et al. 1998; Rosati et al. 2002; Moretti et al. 2003) most of which are the unobscured AGNs familiar from optical and soft X-ray surveys. However, the high-energy spectrum of the CXRB is much harder $(\Gamma \sim 1.4)^{18}$ than that of known AGNs $(\Gamma \sim 1.9)$. Population synthesis models that satisfy both the CXRB spectrum and the observed X-ray number counts versus flux relation $(\log N-\log S$; Comastri et al. 1995; Hasinger et al. 1998; Tozzi et al. 2001) favor absorbed AGNs as a dominant component of the CXRB. In these models, most of the accretion luminosity in the universe is from obscured sources (Hasinger et al. 2000; Fabian \& Iwasawa 1999), which appear to have hard X-ray spectra

\footnotetext{
${ }^{10}$ School of Natural Sciences, Institute for Advanced Study, Princeton, NJ 08540.

${ }^{11}$ MMT Observatory, University of Arizona, 933 North Cherry Avenue, Tucson, AZ 85721-0065.

12 Astronomy Department, University of Texas, Austin, TX 78712.

13 Astronomy Department, Ohio State University, 140 West 18th Avenue, Columbus, OH 43210.

${ }^{14}$ South African Astronomical Observatory, P.O. Box 9, Observatory, 7935, South Africa.

${ }^{15}$ Cerro Tololo Inter-American Observatory, National Optical Astronomy Observatory, Casilla 603, La Serena, Chile.

16 Steward Observatory, University of Arizona, Tucson, AZ 85721.

17 Department of Physics and Astronomy, Ohio University, Athens, OH 45701-2979.

${ }^{18}$ The quantity $\Gamma$ is the photon number index of an assumed power-law continuum such that $N_{E}(E)=N_{E_{0}} E^{\Gamma}$. In terms of a spectral index $\alpha$ from $f_{\nu}=f_{\nu_{0}} \nu^{\alpha}$, we define $\Gamma=(1-\alpha)$.
} 
because circumnuclear gas absorbs low-energy X-rays. X-ray spectral analyses and optical follow-up of faint hard X-ray sources detected by Chandra and XMM-Newton have confirmed this interpretation generally (Brandt et al. 2001; Alexander et al. 2001) but also presented some surprises. Type 2 AGNs are mostly found at $z<1$, and the required space density of such objects must be much greater (Rosati et al. 2002; Alexander et al. 2001; Hornschemeier et al. 2001) than those of standard unabsorbed broad-line AGNs (BLAGNs). For the standard AGN unification model to survive, wherein optical type 1 and type 2 classifications represent different viewing angles on identical objects (Antonucci 1993), it must begin to encompass the population of X-ray absorbed AGNs and its evolution in number density, covering fraction and/or optical depth of absorbers.

Absorption may be increasing with luminosity or redshift (Elvis et al. 1998; Reeves et al. 1997) and could be associated with early circumnuclear starbursts (Guainazzi et al. 2000). The intriguing suggestions of a $z \sim 1$ peak in X-ray-selected (X-S) galaxies (Barger et al. 2002; Tozzi et al. 2001) has been suggested as evidence for an epoch of enhanced activity related to the assembly of massive galaxies (Franceschini, Braito, \& Fadda 2002). At high redshift $(z>4)$, a significant drop-off in the comoving space density of quasars seen in optical (e.g., Schmidt, Schneider, \& Gunn 1995; Warren, Hewett, \& Osmer 1994; Osmer 1982) and radio surveys (Shaver et al. 1996) hints at either the detection of the onset of accretion onto supermassive black holes, or a missed high-redshift population, possibly due to intrinsic absorption. Based on preliminary evidence for constant space densities of X-ray selected quasars beyond a redshift of 2, deep (ROSAT) soft X-ray surveys (Miyaji et al. 2000) have been used to support the latter interpretation. Unfortunately, the sample size is small with only eight quasars beyond a redshift of 3. At these early epochs, higher rates of galaxy interactions and mergers are expected to have triggered nuclear activity in galaxies (e.g., Blain et al. 1999; Osterbrock 1993). As gas-rich protogalaxies grow by merging, they may induce growth in the central black holes. Preliminary models tie together AGN evolution with hierarchical growth of clustering (e.g., Cole \& Kaiser 1989) and galaxy formation models. Wilman, Fabian, \& Nulsen (2000) use the assumption that seed blocks for clustering each contain black holes of mass $1.6 \times 10^{6} M_{\odot}$. During these early high accretion phases, quasars may be self-cloaking, naturally copious producers of metals (Kuraszkiewicz et al. 2002) and dust (Elvis, Marengo, \& Karovska 2002). A truncation of growth in baryonic mass is perhaps achieved by the winddriven gas from the AGN, yielding today's observed black hole/bulge mass correlation (Merrit \& Ferrarese 2001).

At more recent epochs, the bright optically selected (O-S) quasar population appears to fall dramatically between $z \sim 2.5$ and the present, on a timescale of about $2 \mathrm{Gyr}$, quite rapid compared to the fading of galaxy formation in hierarchical clustering models. Strong luminosity evolution dominates the luminosity function (LF) during this epoch across radio, optical, and X-rays, possibly associated with depletion of gas reservoirs by accretion of smaller group companions (Cavaliere \& Vittorini 2000).

\subsection{The Need for the Chandra Multiwavelength Project (ChaMP)}

To understand the formation and evolution of accretion onto supermassive black holes, and its links to galaxy formation, an accurate census of accreting objects is required. Effectively, we must account for the accretion radiation energy density across cosmic time consistent with the mass density of supermassive black holes in the universe (e.g., Yu \& Tremaine 2002; Elvis, Risaliti, \& Zamorani 2002; Mainieri et al. 2002). Galaxies without a dominant active nucleus also produce $\mathrm{X}$-rays from by-products of star formation such as low- and high-mass X-ray binaries (LMXBs and HMXBs), supernova remnants, and hot diffuse gas. The distinction between star formation and AGN-induced emission is a subject of long debate that relates intimately to the above discussion. Progress on the composition of the CXRB, and the accretion and star formation history of the universe all require a wide area, multiwavelength survey with greater sensitivity and completeness than previously achieved, and with reduced bias against absorbed objects.

Both soft X-ray and optical surveys suffer strong selection effects due to intrinsic obscuration and the intervening $\operatorname{Ly} \alpha$ forest. Consistent with the hypothesis that the CXRB is dominated by absorbed AGNs, a substantial population of reddened AGNs is being found in IR (Gregg et al. 2002; Cutri et al. 2001; Masci, Drinkwater, \& Webster 1999; Wilkes et al. 2002) and radio (Becker et al. 1997; Masci et al. 1999; Vester et al. 2001) surveys. While radio surveys are least affected by intrinsic absorption, radio-loud objects constitute a minority of AGNs. Optical classification rests on secondary properties (e.g., emission lines from ionized plasma). By contrast, the primarysignature of accretion is emission from close (10-100 gravitational radii) to the supermassive black hole, which is why only sensitive hard X-ray selection samples all known varieties of AGNs. Furthermore, for Chandra and XMMNewton, sensitivity to emission up to $10 \mathrm{keV}$ (observed frame) can reveal hidden populations of AGNs, including heavily obscured quasars (Norman et al. 2002; Stern et al. 2002). Absorption $\left(\sim 10^{22-23} \mathrm{~cm}^{-2}\right)$ has $10-3000$ times less impact on detection by Chandra $(0.3-8 \mathrm{keV})$ than on ROSAT (0.3$2.4 \mathrm{keV})$. High- $z$ objects can be detected through an even larger intrinsic absorbing column of gas and dust because the observed-frame X-ray bandpass corresponds to higher energy, and hence more penetrating X-rays at the source. ${ }^{19}$ Therefore, optical and X-ray surveys will complement each other, providing a fair census of mass accretion onto black holes at high redshift.

With its low background and tight PSF $\left(\lesssim 1^{\prime \prime}\right.$ FWHM on axis) Chandra is probing the high-energy universe with unparalleled sensitivity and resolution. The Chandra Multiwavelength Project (ChaMP) is a wide-area study of a large sample of serendipitous X-ray sources detected in Chandra archival images. The ChaMP has selected more than 100 Chandra images, in which we will detect several thousand serendipitous (nontarget) extragalactic sources. A first X-ray catalog of these sources and early results are published in accompanying papers (Kim et al. 2003a, 2003b). The ChaMP AGN sample includes faint nearby AGNs, absorbed quasars to $z \sim 3$ and unobscured AGNs to $z \sim 5$, populating new regions of $L-z$ space, and providing a broad census of the population that will reap statistically significant samples for rare source types. The two $0.1 \mathrm{deg}^{2}$ Chandra $1 \mathrm{Ms}$ Deep Fields probe far too little volume at low redshift where many of the absorbed AGNs probably reside (Barger et al. 2001; Fiore et al. 2000)

\footnotetext{
19 The observed-frame, effective absorbing column is $N_{\mathrm{H}}^{\text {eff }} \sim N_{\mathrm{H}} /(1+z)^{2.6}$ (Wilman \& Fabian 1999).
} 
and contain few bright examples to easily characterize these sources. The ChaMP will effectively bridge the gap between flux limits achieved with the Chandra deep field (CDF) observations and those of past surveys. The flux regime where the ChaMP has greatest sky area $\left(10^{-14} \operatorname{ergs} \mathrm{cm}^{-2} \mathrm{~s}^{-1}\right)$ coincides with the break between power-law slopes fitting the hard (2-10 keV) $\log N-\log S$ (Rosati et al. 2002). Furthermore, as shown in Figure 1 of Kim et al. (2003b), a gap in coverage remains between deep ASCA and Chandra surveys near the break. The wide-area and medium depth of Chandra fields included in the ChaMP will span these fluxes with good statistics.

A number of new high Galactic latitude serendipitous surveys are underway both with XMM-Newton and Chandra. The XMM-Newton Survey Science Center (Watson 2001) targets several flux ranges, including a medium sensitivity survey $\left.(f 90.5-4.5 \mathrm{keV})>2 \times 10^{-14} \mathrm{ergs} \mathrm{cm}^{-2} \mathrm{~s}^{-1}\right)$ with first results described by Barcons et al. (2002). The XMM-Newton EPIC instrument covers a large $\left(\sim 15^{\prime}\right.$ radius $)$ field of view and has sensitivity out to higher energies than Chandra. However, given the higher background and larger PSF of XMM-Newton $\left(5^{\prime \prime}-7^{\prime \prime}\right.$ FWHM), the minimum detectable flux for an on-axis point source with a typical AGN spectrum is similar. For cross-correlation to faint sources in other wavebands, the tight Chandra PSF is a great advantage.

While ChaMP emphasizes study of the nature and evolution of active galaxies, as sketched above, it will also provide to the scientific community well-defined wide-area survey products suitable for detailed multiwavelength studies of quasars, stars, star formation, galaxies, clusters of galaxies, and clustering.

We have chosen to analyze a subsample of six ChaMP fields for this paper as an example of what can be expected from the full ChaMP sample. After describing in $\S 2$ the overall ChaMP field selection process, we briefly describe the processing and analysis of the X-ray images in $\S 3$, which is detailed in our companion X-ray paper (Kim et al. 2003a). We then outline our ongoing optical imaging campaign (§4), X-ray/optical source matching ( $(5)$, and optical spectroscopy $(\S 6)$. We describe overall sample results in $\S 7$, with separate emphasis on AGNs, galaxies, and clusters of galaxies. Prospects and plans for ChaMP science are sketched in $\S 9$. We discuss selected individual objects in each of the six fields in the Appendix. Throughout this paper, we assume $H_{0}=$ $70 \mathrm{~km} \mathrm{~s}^{-1} \mathrm{Mpc}^{-1}, \Omega_{\Lambda}=0.7$, and $\Omega_{M}=0.3$.

\section{FIELD SELECTION AND X-RAY IMAGE PROCESSING}

Our field selection and X-ray image processing strategies are detailed in the companion X-ray paper of Kim et al. (2003a), Briefly, fields are restricted to high Galactic latitudes $\left(|b|>20^{\circ}\right)$. We omit a handful of pointings from other serendipitous surveys like the Chandra Deep Fields and exclude fields with large bright optical or X-ray sources. This selection yields 146 ACIS exposures in cycles 1 and 2, with Chandra exposure times ranging from 2 to $190 \mathrm{ks}$ and covering about $14 \mathrm{deg}^{2}$. The sky placement of these fields is shown in an equatorial Aitoff projection in Figure 1. The full field list is public, available at the ChaMP Web site. ${ }^{20}$

The fields studied in this pilot paper include six early (cycle 1) fields listed in Table 1 for which we have already

\footnotetext{
${ }^{20}$ See http://hea-www.harvard.edu/CHAMP.
}

reduced and calibrated both archival X-ray images and photometric NOAO $4 \mathrm{~m} /$ Mosaic imaging. These six fields represent about $12 \%$ of ChaMP fields of similar exposure time or greater. Four of the six fields have clusters as their intended Chandra targets. We do not include the intended (PI) targets in our analysis, their presence may bias the sample of source types analyzed here. The large number of fields in the full ChaMP and the variety of PI target types allow important tests for possible sample biases. While an excess of point sources in cluster fields has been claimed anecdotally (Cappi et al. 2001; Martini et al. 2002), no significant difference is found using the larger sample of Chandra images (29 with clusters and 33 without) in Kim et al. (2003b).

\section{X-RAY IMAGE PROCESSING AND ANALYSIS}

We have used Chandra archival data reprocessed (in 2001 April) at CXC. ${ }^{21}$ The foundation of the ChaMP rests on our own Chandra image analysis pipeline XPIPE (Kim et al. 2003a), built mainly with CIAO tools ${ }^{22}$ and designed to uniformly and carefully screen and analyze the X-ray data. Details of XPIPE can be found in our X-ray companion paper, Kim et al. (2003a). Briefly, XPIPE screens high particle background periods, cosmic rays, and hot pixels from each ACIS CCD exposure. Wavelet transform detection is performed (using wavdetect; Freeman et al. 2002) at seven scales from 0.5 to $32^{\prime \prime}$, thus accommodating the Chandra PSF variation as a function of off-axis angle from the mirror axis. We select a significance threshold parameter of $10^{-6}$ per pixel, which corresponds to about one spurious pixel detection per $\mathrm{CCD}$. We have confirmed this rate by an extensive simulation showing that the number of spurious sources detected is always less than this rate. An additional flux criterion of signal to noise $(\mathrm{S} / \mathrm{N})$ greater than 2 from our X-ray photometry further decreases the number of spurious sources in the sample. We avoid spurious sources near detector edges by generating an exposure map for each CCD with the appropriate aspect histogram and then applying a minimum exposure threshold of $10 \%$ to all pixels included for analysis.

The absolute celestial positions of sources detected with wavdetect are accurate to about $1^{\prime \prime}$, which is the specified absolute position accuracy for the Chandra observatory. ${ }^{23}$ To check for and minimize any systematic offset in the absolute source positions returned by wavdetect, the positions of X-ray sources are correlated to optical sources in the field. The average position offset from X-ray sources to matched optical sources is then applied to each X-ray source position. This procedure is described in more detail in $\S 5.1$.

We visually inspect all the $\mathrm{X}$-ray images to validate sources and flag them for contamination (by hot pixels, readout streaks, bad bias values) or excessive uncertainty due to contamination of source or background regions. Throughout this paper and for ChaMP statistics, the target sources are flagged and excluded from analysis in all fields. Source catalogs and FITS images are being made publicly available, with details at the ChaMP Web site.

At the X-ray source positions derived from wavdetect, we perform aperture photometry. We use a circular source

\footnotetext{
${ }^{21}$ CXCDS versions R4CU5UPD14.1 or later, along with ACIS calibration data from the Chandra CALDB $2.0 \mathrm{~b}$.

${ }^{22}$ See http://asc.harvard.edu/ciao.

${ }^{23} \mathrm{See} \mathrm{http} / / /$ asc.harvard.edu/cal/ASPECT for a detailed discussion of Chandra aspect solutions.
} 


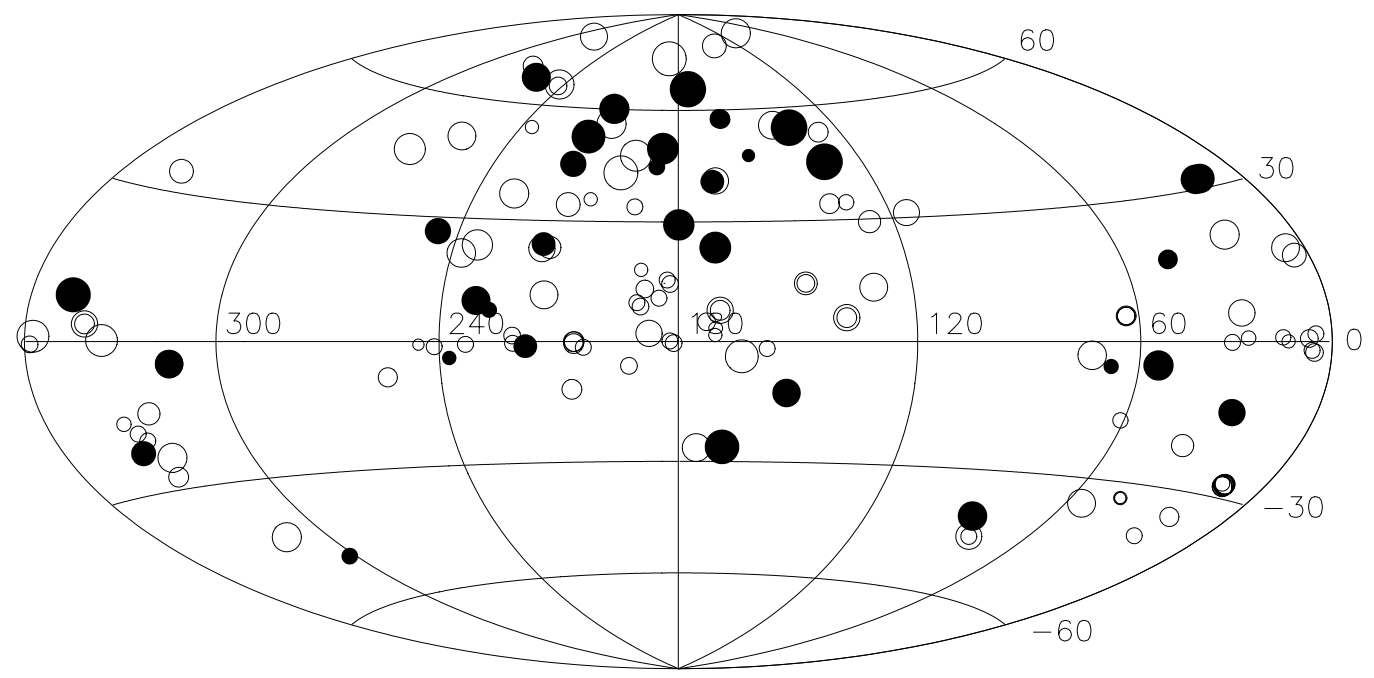

Fig. 1. - Location of all 137 ChaMP high-latitude $\left(|b|>20^{\circ}\right)$ fields is shown in an Aitoff equatorial projection, with R.A. and Decl. in decimal degrees. Filled circles represent ACIS-I at the aim point, and open circles ACIS-S. The average area for ChaMP-selected Obs. IDs corresponds to 5.8 CCDs turned on, each of which is 8.3 on a side. Point size crudely indicates Chandra exposure times, varying from 2 to $190 \mathrm{ks}$.

aperture whose radius encompasses $95 \%$ of deposited energy, determined at $1.5 \mathrm{keV}$, but restrict this radius to the range $3^{\prime \prime}<r_{\text {ap }}<40^{\prime \prime}$. We use a background annulus extending from 2 to 5 times $r_{\text {ap }}$, excluding flux within $r_{\text {ap }}$ of any other detected sources in the annulus. We perform photometry in three energy bands: soft $(S ; 0.3-2.5 \mathrm{keV})$, hard $(H ; 2.5-8 \mathrm{keV})$, and broad $(B ; 0.3-8 \mathrm{keV})$. We calculate net count rates using the effective exposure and vignetting factors both at the source and background regions.

Only sources with $\mathrm{S} / \mathrm{N}>2$ in at least one of the bands $B, S$, or $H$ are included for analysis here. ${ }^{24}$ Flux is calculated from the net count rate in the $B$, or if necessary from another ( $H$ or $S$ ) band to satisfy our $\mathrm{S} / \mathrm{N}$ criterion. We assume a power law of photon index $\Gamma=1.4$ absorbed by a neutral Galactic column density $N_{\mathrm{H}}$ taken from Dickey \& Lockman (1990) for

${ }^{24} \mathrm{~S} / \mathrm{N}$ is calculated from $\sigma=\left(\sigma_{\mathrm{tot}}^{2}+R^{2} \sigma_{\mathrm{bkg}}^{2}\right)^{1 / 2}$, where $\sigma_{\text {tot }}$ is the error in the total number of counts in the source aperture, and $\sigma_{\mathrm{bkg}}$ is the error on counts in the source-excised background aperture; $R$ is the ratio of areas of source and background regions. The $\mathrm{S} / \mathrm{N}$ criterion is a flux, not a detection criterion, since detection is based on probability in wavdetect. Sources with $\mathrm{S} / \mathrm{N}=2$ can be easily detected in many cases, since the background rates are typically 1 broadband $(0.5-10 \mathrm{keV})$ count per 40 pixels in $100 \mathrm{ks}$ (CXC v4.0 2001). the Chandra aim point position on the sky. The effect of varying the source model between reasonable power-law slopes from $\Gamma=1.0$ to 2.5 causes (for $N_{\mathrm{H}}^{\mathrm{Gal}}=3 \times 10^{20} \mathrm{~cm}^{-2}$ ) an increase in the derived $f_{\mathrm{X}}(0.5-2 \mathrm{keV})$ of $\sim 12 \%$ for FI chips, and $6 \%$ for BI chips.

The input bandpass energy range is passed to PIMMS (Mukai 1993; v3.2d, which includes Chandra cycle 4 effective area curves) along with the adjusted count rate and spectral model parameters to calculate a deabsorbed flux $f_{\mathrm{X}}$ in the $0.5-2 \mathrm{keV}$ band, since this bandpass is most useful for comparison to numerous published results from ROSAT, $X M M-N e w t o n$, and Chandra. The error in $f_{\mathrm{X}}$ is computed by applying the same counts-to-flux conversion factor to the error in counts, increased by a further $15 \%$ to qualitatively account for errors induced by the spectral model assumptions. These results are listed on-line along with other quantities described below.

For every source, we wish to calculate an effective on-axis ACIS-I hardness ratio,

$$
\mathrm{HR}=\frac{H-S}{H+S},
$$

TABLE 1

Chandra FIELDS

\begin{tabular}{|c|c|c|c|c|c|c|c|}
\hline Obs. ID & PI Target & $\begin{array}{c}\text { Exposure }^{\mathrm{a}} \\
(\mathrm{ks})\end{array}$ & ACIS CCDs ${ }^{b}$ & $\begin{array}{l}\text { R.A. } \\
(\mathrm{J} 2000)^{\mathrm{c}}\end{array}$ & $\begin{array}{c}\text { Decl. } \\
(\mathrm{J} 2000)^{\mathrm{c}}\end{array}$ & UT Date & $\begin{array}{l}\text { Galactic } N_{\mathrm{H}} \\
\left(10^{20} \mathrm{~cm}^{-2}\right)\end{array}$ \\
\hline $536 \ldots$ & MS $1137.5+6625$ & 114.6 & 012367 & 114023.3 & +660842.0 & 1999 Sep 30 & 1.18 \\
\hline 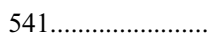 & V1416+4446 & 29.8 & 01237 & 141628.8 & +444640.8 & 1999 Dec 02 & 1.24 \\
\hline 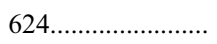 & LP 944-20 & 40.9 & 23678 & 033934.7 & -352550.0 & 1999 Dec 15 & 1.44 \\
\hline $861 \ldots \ldots$ & Q2345+007 & 65.0 & 23678 & 234819.6 & +00 5721.1 & 2000 Jun 27 & 3.81 \\
\hline 914........................... & CL J0542-4100 & 48.7 & 01237 & 054250.2 & $\begin{array}{llll}-41 & 00 & 06.9\end{array}$ & 2000 Jul 26 & 3.59 \\
\hline 928 & MS 2137-2340 & 29.1 & 23678 & 214012.7 & -233927.0 & 1999 Nov 18 & 3.57 \\
\hline
\end{tabular}

${ }^{a}$ Effective screened exposure time for the on-axis chip. BI chips 5 and 7 generally have lower screened exposures since they are more susceptible to solar flares.

${ }^{b}$ The ACIS CCD chips used in the observation, with the aim point chip in italics. The ACIS has 10 chips total, of which CCDs 5 and 7 are back-illuminated. Due to telemetry limitations, at most six chips can be read out.

${ }^{c}$ Nominal target position, not including any Chandra pointing offsets. Units of right ascension are hours, minutes, and seconds, and units of declination are degrees, arcminutes, and arcseconds. 
that may be fairly compared among sources to study their X-ray properties even in the low counts regime. Many (especially weak) sources have derived $H$ or $S$ counts that are formally less than $2 \sigma$, or even negative. Naive inclusion of these yields nonsensical HR-values outside the physical range from -1 to 1 . Therefore, we first replace such counts by a $2 \sigma$ upper limit. This yields for HR either a "detection" (when both $H$ and $S$ counts are greater than $2 \sigma$ ), an upper limit (when $H<2 \sigma_{H}$ ), a lower limit (when $S<2 \sigma_{S}$ ), or very rarely an indeterminate value (both).

Mirror reflectance changes as a function of energy and of the angle $\theta$ between the source and Chandra's aim point, causing energy-dependent vignetting. A different energy dependence arises from the detecting $\mathrm{CCD}$ - the quantum efficiency of back-side-illuminated (BI) versus front-side-illuminated (FI) chips. Therefore, for an identical source spectrum, different HR-values result, depending on $\theta$ and chip. To correct for this, we perform a second adjustment of $H$ and $S$ counts, normalizing them to refer uniformly to an on-axis source falling on an FI chip. To do this, we have modified the PIMMS code to include vignetting. Where the integration of source model is convolved with the instrument effective area (EA), we incorporate an extra multiplicative factor - the percentage of the on-axis EA as a function of photon energy and $\theta$. We interpolate between analytic fits (courtesy Diab Jerius) to EA as a function of $\theta$ from $0^{\prime}$ to $30^{\prime}$, performed for four energies $(0.49,1,1.48,2.02,2.99$, and $6.4 \mathrm{keV})$. Similar curves are shown in the CXC Proposer's Guide Rev. 4.0 (CXC v4.0 2001).

To derive the corrected hardness ratio $\mathrm{HR}_{0}$, we first convert counts to deabsorbed flux in each band using our standard source model, the source $\theta$ and chip. Using the same source model, we then convert the derived flux back counts at $\theta=0$ on an FI chip. The resulting corrections in $\mathrm{HR}_{0}$ do not depend strongly on the assumed $\Gamma$; the difference in the mean $\mathrm{HR}_{0}$ derived assuming $\Gamma=0.5$ and 3.0 , is only $0.02(3 \%)$ out to $15^{\prime}$ off-axis.

We have performed a series of simulations to derive the expected $\mathrm{HR}_{0}$-values for high $\mathrm{S} / \mathrm{N}$ sources across a grid of $\Gamma$ and $N_{\mathrm{H}}$-values, as shown in Figure 2 . A comparison to the median corrected value of $\overline{\mathrm{HR}_{0}}=-0.5$ for all sources detected in both $H$ and $S$, and assuming no absorption, implies $\Gamma \sim 1.4$.

Finally, if at least one of $H$ and $S$ are significant, then we calculate errors for $\mathrm{HR}_{0}$ from the $H$ and $S$ counts (or limits) as

$$
\sigma_{\mathrm{HR}}=\frac{2}{(H+S)^{2}} \sqrt{S^{2} \sigma_{H}^{2}+H^{2} \sigma_{S}^{2}} .
$$

\section{OPTICAL IMAGING}

Optical imaging is crucial to provide optical fluxes, preliminary source classification, and accurate centroiding for spectroscopic follow-up. Optical centroids supersede X-ray centroids for extended or multiple objects, for cluster galaxies, or for Chandra sources with large off-axis angles and hence less accurate X-ray positions. Multiband colors can provide source classifications and photometric redshifts for the majority of fainter ChaMP sources $\left(r^{*}>22\right)$ for which highquality spectra are more difficult to obtain.

\subsection{Exposure Times}

For every ChaMP field, we scale our optical exposure times to the Chandra X-ray exposure times, to provide a uniform sensitivity to X-ray/optical flux ratios. Our color-limited
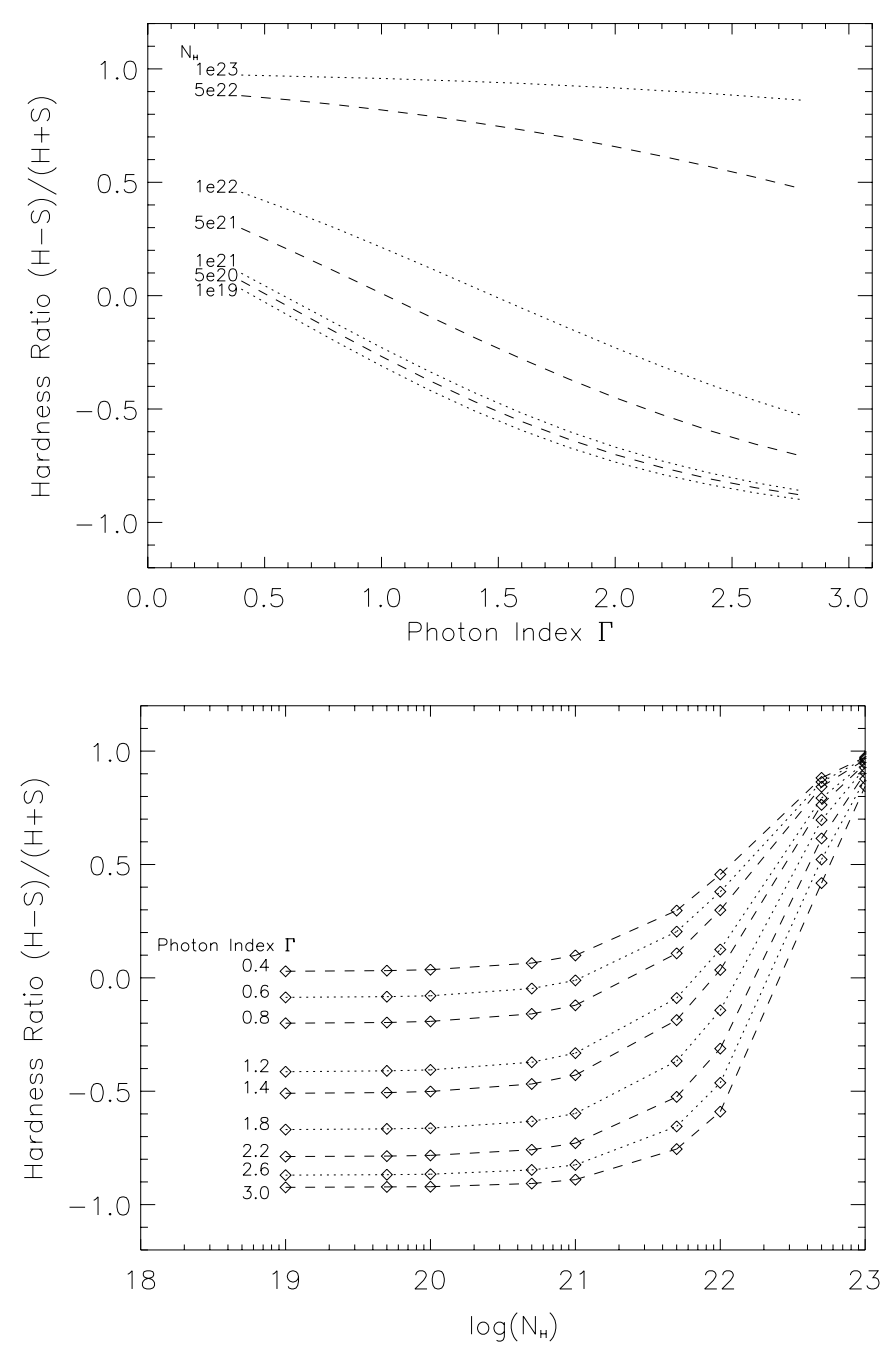

FIG. 2.- Simulated on-axis FI chip hardness ratios for high $\mathrm{S} / \mathrm{N}$ sources. Top. The family of curves show the resulting HR for different $(z=0)$ absorbing columns as a function of power-law photon index $\Gamma$. Bottom. The family of curves show the resulting HR for different values of power-law photon index $\Gamma$ as a function of absorbing column.

survey thus minimizes optical telescope usage, while accessing for every field a similar fraction of every object type. To convert X-ray counts to flux, we first assume $80 \%$ of the exposure time remains after cleaning high particle background periods. $^{25}$ The low background and small PSF of Chandra allows high confidence detection of point sources with as few as 4-5 photons, but to reduce uncertainties on $f_{\mathrm{X}}$ and thereby improve object class discrimination, we adopt 10 counts as the ChaMP minimum detection limit. We convert the detectable number of counts for each Chandra field to an X-ray flux limit and then scale the corresponding optical magnitude limit to include more than $90 \%$ of ROSAT AGNs even at the X-ray flux limit, based on 1448 ROSAT-detected quasars from Yuan et al. (1998). The typical resulting magnitude limit as a function of Chandra exposure time $T$ can be expressed as $V=14.17+$ $2.5 \log T$. This criterion $\left[\log \left(f_{\mathrm{X}} / f_{V}\right)<0.9\right]$ should include larger fractions of other source types with brighter relative optical emission like stars and most galaxies. Sources that

\footnotetext{
${ }^{25}$ Since X-ray photons are tagged with arrival times, high background periods can be excised.
} 
are relatively weak optically compared to ROSAT quasars will have a lower completeness and a brighter effective limiting magnitude in our survey (e.g., sources suffering from substantial optical extinction). Details of our optical imaging for these six fields are presented in Table 2 . The resulting desired limits range from $V$ magnitudes [note that $V \sim\left(g^{\prime}+r^{\prime}\right) / 2$ ] of 21 to 26 with a median of $23.5 \mathrm{mag}$ for all ChaMP fields. However, to achieve reasonable limits on the amount of required optical imaging time, we limit our maximum desired optical magnitude limit to $V \sim 25$ in any field.

NOAO $4 \mathrm{~m}$ imaging with the Mosaic CCD cameras (Muller et al. 1998) is key to the ChaMP, since it provides adequate depth, spatial resolution $\left(\sim 0\right.$ ".26 pixel $\left.{ }^{-1}\right)$, and a large field of view $\left(36^{\prime} \times 36^{\prime}\right)$ over the full Chandra FoV. For shallower northern fields, for secondary calibration of deep imaging from nonphotometric $4 \mathrm{~m}$ nights, and for imaging bright objects within deep fields, the ChaMP also uses the FLWO $1.2 \mathrm{~m}$ with the 4shooter CCD on Mount Hopkins (0"32 pixel $^{-1}$, 22' field). In this paper, we report only deep $4 \mathrm{~m}$ fields observed in photometric conditions.

We dither the $4 \mathrm{~m}$ / Mosaic pointings to allow for better sky flat construction, averaging over defects, cosmic-ray removal, and gap coverage. While the optical data reduction is conceptually straightforward, it is in practice complicated by the size of the data set (285 MB per image), and by the need to interactively manage bad pixel flagging. After photometric analysis, our final reduced images are stored in (short) integer format, which reduces their size by a factor of 2 , with less than $1 \%$ effect on measured magnitudes.

\subsection{Filter Choice}

The ChaMP uses Sloan Digital Sky Survey (SDSS) $g^{\prime}, r^{\prime}$, and $i^{\prime}$ filters (Fukugita et al. 1996), whose steep transmission cutoffs improve object classification and photometric redshift determination (Gonzalez \& Maccarone 2002; Richards et al. $2001)$. While $f_{\mathrm{X}} / f_{\text {opt }}$ is clearly our primary discriminant of
AGNs from stars, at least two optical filters are required to also constrain stellar spectral class. Still, with just two filters, high- $z$, reddened, or obscured AGNs may share the stellar locus with M dwarfs (Lenz et al. 1998), so we chose three filters to separate such quasars from stars because these types of AGNs represent a prime science goal of the ChaMP. Furthermore, it has already been demonstrated that AGNs of redshift $3.5<z<5$ can be constrained to $\Delta z \sim 0.2$ with SDSS $g^{\prime}, r^{\prime}$, and $i^{\prime}$ colors (Richards et al. 2001). Since $g^{\prime}$ and $r^{\prime}$ also bracket the $4000 \AA$ break in $z>0.8$ cluster ellipticals, this aids distance estimation and cluster membership evaluation. The bright $\lambda 5577$ night sky line falls between the $g^{\prime}$ and $r^{\prime}$ filters, allowing fainter limits to be achieved in shorter integration times. Finally, someday the need for NOAO northern imaging follow-up will be relieved by the SDSS itself for the shallower ${ }^{26}$ ChaMP fields. The huge database of SDSS magnitudes and colors help with deeper fields and core science as well by offering (1) bootstrap calibration of deep ChaMP imaging observed in nonphotometric conditions (2) calibration and testing of accurate photometric redshifts (Richards et al. 2001) and source classification (Newberg 1999) (3) detailed comparisons between SDSS optical and ChaMP X-ray sample selection.

\subsection{Optical Image Reduction}

The reduction of the raw optical CCD images is primarily done using standard techniques in IRAF. ${ }^{27}$ The Mosaic cameras utilize the multiextension FITS format, allowing the

\footnotetext{
${ }^{26}$ The SDSS achieves $\mathrm{S} / \mathrm{N} \sim 10$ to $g^{\prime} \lesssim 22 \mathrm{mag}$ at best (Richards et al. 2001).

${ }^{27}$ IRAF is distributed by the National Optical Astronomy Observatory, which is operated by the Association of Universities for Research in Astronomy, Inc., under cooperative agreement with the National Science Foundation.
}

TABLE 2

Optical IMAging

\begin{tabular}{|c|c|c|c|c|c|c|c|c|c|c|}
\hline Obs. ID & $E(B-V)$ & Telescope & UT Date & Filter & Dithers & $\begin{array}{c}\text { Exposure } \\
\text { (total s) }\end{array}$ & $\begin{array}{c}\text { Air Mass } \\
\text { (Mean) }\end{array}$ & $\begin{array}{c}\mathrm{FWHM}^{\mathrm{a}} \\
(\operatorname{arcsec})\end{array}$ & $\begin{array}{l}m_{\mathrm{TO}}^{\mathrm{b}} \\
\text { Limit }\end{array}$ & $\begin{array}{l}m_{5 \sigma^{\mathrm{c}}} \\
\text { Limit }\end{array}$ \\
\hline \multirow[t]{3}{*}{536} & \multirow[t]{3}{*}{0.0131} & \multirow[t]{3}{*}{ KPNO $4 \mathrm{~m}$} & \multirow[t]{3}{*}{2000 Jun 12} & $g^{\prime}$ & 2 & 1200 & 1.39 & 2.2 & 22.875 & 23.9 \\
\hline & & & & $r^{\prime}$ & 2 & 1200 & 1.43 & 2.0 & 22.875 & 24.2 \\
\hline & & & & $i^{\prime}$ & 2 & 1200 & 1.48 & 1.9 & 22.625 & 23.8 \\
\hline \multirow[t]{3}{*}{$541 \ldots \ldots \ldots \ldots \ldots \ldots \ldots$} & \multirow[t]{3}{*}{0.008} & \multirow[t]{3}{*}{ KPNO $4 \mathrm{~m}$} & \multirow[t]{3}{*}{2000 Jun 12} & $g^{\prime}$ & 2 & 1000 & 1.33 & 1.9 & 22.875 & 23.9 \\
\hline & & & & $r^{\prime}$ & 1 & 500 & 1.40 & 2.0 & 22.125 & 23.7 \\
\hline & & & & $i^{\prime}$ & 1 & 500 & 1.50 & 1.7 & 22.125 & 23.5 \\
\hline \multirow[t]{3}{*}{$624 \ldots \ldots \ldots \ldots \ldots \ldots \ldots \ldots$} & \multirow[t]{3}{*}{0.014} & \multirow[t]{3}{*}{ CTIO $4 \mathrm{~m}$} & \multirow[t]{3}{*}{2000 Sep 28} & $g^{\prime}$ & 3 & 810 & 1.05 & 1.2 & 24.875 & 25.9 \\
\hline & & & & $r^{\prime}$ & 3 & 630 & 1.01 & 1.1 & 24.125 & 25.3 \\
\hline & & & & $i^{\prime}$ & 3 & 720 & 1.00 & 1.1 & 23.375 & 24.4 \\
\hline \multirow[t]{3}{*}{$861 \ldots \ldots \ldots \ldots \ldots \ldots \ldots \ldots$} & \multirow[t]{3}{*}{0.0246} & \multirow[t]{3}{*}{ CTIO $4 \mathrm{~m}$} & \multirow[t]{3}{*}{2000 Sep 28} & $g^{\prime}$ & 3 & 1260 & 1.23 & 1.4 & 24.625 & 25.8 \\
\hline & & & & $r^{\prime}$ & 3 & 1080 & 1.17 & 1.4 & 24.375 & 25.3 \\
\hline & & & & $i^{\prime}$ & 3 & 1170 & 1.17 & 1.1 & 23.375 & 24.3 \\
\hline \multirow[t]{3}{*}{914} & \multirow[t]{3}{*}{0.0383} & \multirow[t]{3}{*}{ CTIO 4 m } & \multirow[t]{3}{*}{2000 Sep 28} & $g^{\prime}$ & 3 & 990 & 1.04 & 1.2 & 25.125 & 26.1 \\
\hline & & & & $r^{\prime}$ & 3 & 810 & 1.06 & 1.2 & 24.375 & 25.4 \\
\hline & & & & $i^{\prime}$ & 3 & 900 & 1.09 & 1.2 & 23.375 & 24.2 \\
\hline \multirow[t]{3}{*}{ 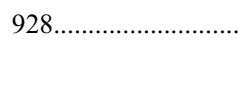 } & \multirow[t]{3}{*}{0.051} & \multirow[t]{3}{*}{ CTIO $4 \mathrm{~m}$} & \multirow[t]{3}{*}{2000 Sep 28} & $g^{\prime}$ & 3 & 900 & 1.01 & 1.6 & 24.375 & 25.6 \\
\hline & & & & $r^{\prime}$ & 3 & 720 & 1.02 & 1.3 & 24.125 & 25.2 \\
\hline & & & & $i^{\prime}$ & 3 & 810 & 1.05 & 1.0 & 23.375 & 24.3 \\
\hline
\end{tabular}

${ }^{\text {a }}$ FWHM of point sources in final stacked images.

b Turnover magnitude limit at $\sim 90 \%$ completeness, using 0.25 mag bins before extinction correction, as described in the text.

${ }^{\mathrm{c}}$ Magnitude limit for a $\sim 5 \sigma$ detection. 
eight individual CCD images to be treated as a single image for most reduction purposes. The IRAF package mscred (Valdes 2002) is used to properly handle the multiextension FITS images and to simplify the data reduction. Although an overview is presented here, a detailed reduction walkthrough is available ${ }^{28}$ for the NOAO Deep Wide Field Survey (NDWFS) data (Jannuzi \& Dey 1999) using the same instruments.

Standard calibration images are taken at the telescope (bias, dome flats, etc.) excluding twilight sky flats. We construct a master super-sky flat by combining multiple object frames, thereby rejecting real objects in the frame and leaving us with a high S/N image of "blank" sky. These improve the flat-field correction provided by conventional dome flats, mostly by accounting for differences in each filter between the night-sky and our dome lamp color. Before the super-sky flat can be made, images from the KPNO $4 \mathrm{~m}$ also require subtraction of a pupil ghost caused by light back-scattered from the telescope optics, which affects primarily the inner four CCDs. Because the pupil ghost changes with the amount of light entering the telescope, its strength varies with the filter used, and with ambient light from the moon or bright stars near the field of view. For this reason we create a template pupil ghost separately for each filter, taking care to exclude images with extremely bright saturated objects near the center of the field of view. Once a template pupil has been generated, it must be scaled and subtracted from individual object frames in each filter, a process that is now largely automated.

\subsection{Astrometry and Photometry}

We first derive $\left(\sim 0{ }^{\prime \prime} 3 \mathrm{rms}\right)$ astrometry from our optical imaging to enable accurate X-ray source matches, and optimal positioning of spectroscopic fibers or slits. The (J2000) position of the optical sources are referenced to the Guide Star Catalog II. ${ }^{29}$ Final positions used by the ChaMP for all matched sources are the average of positions measured from all optical filters in which an object is detected.

We use SExtractor (Bertin \& Arnouts 1996) to detect sources and measure their positions and magnitudes. A first run of SExtractor is used to measure the mode $\Gamma$ of the FWHM of stellar objects in each image. For this step, our filters first remove objects that are unreliable (any with processing error flag greater than 3-cosmic rays, spurious blended, or contaminated sources, or sources near a chip edge), sources that are too faint (typically less than $2 \times 10^{4}$ net counts) or too bright (typically more than $2 \times 10^{5}$ counts), and extended sources (SExtractor stellarity < median). For program fields, we typically use a minimum detection threshold (SExtractor detect thresh) of $0.8 \sigma$ above background per pixel, and require a minimum grouping (detect_minarea) of $\Gamma^{2} / 4$ pixels at this threshold for a detection. Since we are cross-correlating to X-ray sources - rare on the sky compared to optical sourceswe are somewhat more concerned with completeness than with spurious source rejection. From detailed comparisons of dithered and deep stacked images of the same fields, we find that these parameter settings forge a good compromise between detecting most real sources, but not too many spurious sources.

\footnotetext{
${ }^{28}$ See http://www.noao.edu/noao/noaodeep/ReductionOpt/frames.html.

${ }^{29}$ The Guide Star Catalogue-II is a joint project of the Space Telescope Science Institute and the Osservatorio Astronomico di Torino.
}

A second run of SExtractor measures three instrumental magnitudes: one from a circular aperture with diameter FWHM (for highest S/N; see Howell 1989), another with 4 times the FWHM (which we find encompasses 95\% flux for point sources), and finally SExtractor's mag_auto. The latter is a elliptical aperture magnitude (a là Kron 1980) that is robust to seeing variations, as described in Nonino et al. (1999). Following the convention of the early data release of the SDSS quasar catalog (Schneider et al. 2002), we present the optical photometry here as $g^{*}, r^{*}$, and $i^{*}$ since the SDSS photometry system is not yet finalized and the NOAO filters are not a perfect match to the SDSS filters. Standard stars calibrated for the SDSS are available from Smith et al. (2002a) but are generally too few per field to practically allow for a complete photometric calibration, especially given the long readout times for the Mosaic. To facilitate the use of the many standard stars listed by Landolt (1992), we transform them to the SDSS photometric system using Fukugita et al. (1996) to derive our nightly photometric solutions. In the standard star fields, we find standard stars automatically by positional matching of accurate coordinates for Landolt standards ${ }^{30}$ to SExtractor source positions, using a high $(\sim 7 \sigma)$ SExtractor detection threshold to provide unambiguous detections.

We merge instrumental photometry results from each filter into a multicolor file by positional matching using a search radius of $1^{\prime \prime}$. If multiple matches are found within this radius, the closest match is retained. If no matching detection is found in some filter, the rms of the background for that filter at that object's position is recorded for later conversion to a limiting magnitude. In these six fields, our merged catalog of Mosaic photometry contains about 343,000 objects with flags of less than 4 indicating good photometry in at least one band.

We then perform photometric calibration of the standard stars for each night via a two-step multilinear method (Hardie 1962), adapted from an implementation written in IDL by James Higdon. For a given night, for the instrumental magnitude $v$ in each filter, we solve the linear equation

$$
v_{0}-v=\left(k_{0}+k_{1} X\right)+e\left(c_{0}\right)+z,
$$

where $v_{0}$ and $c_{0}$ are the standard star's cataloged magnitude and color, respectively, $e$ is the color coefficient, $z$ is a zeropoint correction, and $k_{0}$ and $k_{1}$ are extinction coefficients. We iteratively solve for these four coefficients, first freezing $k_{0}$ and $k_{1}$ and solving for $e$ and $z$, then freezing $e$ and $z$ and solving for $k_{0}$ and $k_{1}$, until the solution converges (typically 3 or 4 iterations). We then delete measurements with residuals exceeding $3 \sigma$, and repeat the solution until no such residuals remain.

Similarly, for each color $c$ we iteratively solve the linear equation

$$
c_{0}=e\left(c+k_{0}+k_{1} X\right)+z
$$

for the four coefficients $e, z, k_{0}$ and $k_{1}$, deleting measurements with residuals exceeding $3 \sigma$ as before. We tested the final results from our procedure against that of Harris, Fitzgerald, \& Reed (1981) as implemented in the IRAF photcal procedure and find results identical to within the

\footnotetext{
${ }^{30}$ A. Henden, 2002, ftp://ftp.nofs.navy.mil/pub/outgoing/aah/phot/ landolt.std.
} 
errors. Our calibrations produce $e, z, k 0, k 1$ coefficients for each of $g^{*}, r^{*}, i^{*},\left(g^{*}-r^{*}\right)$, and $\left(r^{*}-i^{*}\right)$. Since these calibrations for these NOAO filters have not been published previously, we list them in Table 3 as a reference. The final transformations are applied to the standard stars to derive rms residuals for each night for $g^{*}, r^{*}, i^{*},\left(g^{*}-r^{*}\right)$, and $\left(r^{*}-i^{*}\right)$. These are listed in Table 3 for each night. The nightly transformations applied to our program objects are

$$
\begin{gathered}
c^{*}=e\left(c+k_{0}+k_{1} X\right)+z, \\
v^{*}=v+\left(k_{0}+k_{1} X\right)+e\left(c^{\prime}\right)+z,
\end{gathered}
$$

where $c^{*}$ and $v^{*}$ are the final calibrated color and magnitude, respectively.

For objects not detected in any optical filter, we assign a magnitude limit $m_{5 \sigma}$ corresponding to a flux of $5 \sigma$ detection where $\sigma$ is the background rms at that position. Starting from the standard CCD equation, we find

$$
m_{D \sigma}=-2.5 \log \left(\frac{D \Gamma \sigma}{T}\right)+z
$$

Here $D$ is the desired $\mathrm{S} / \mathrm{N}$. The rms noise at the position of an object on the CCD is denoted as $\sigma ; \Gamma$ is again the FWHM in pixels, $T$ is the total exposure time, and $z$ indicates that the photometric calibration is applied, for which we assume the mean color for objects in the field. This limiting point source magnitude is listed for each field in Table 2 for the median background $\mathrm{rms}$ in the field. Objects at these magnitudes are not detected with high completeness. From our comparisons of individual dithered and deep stacked images, we find that the magnitude where the number counts peak in a differential (0.25 mag bin) number counts histogram corresponds approximately to $90 \%$ completeness in the magnitude range $20-25$. This turnover magnitude $m_{\text {TO }}$ is typically about 1 mag brighter than the $5 \sigma$ limiting magnitude. Both magnitude limits are listed in Table 2.

We perform several sanity checks on the final photometry. First, we plot color-color diagrams against a crude mean stellar locus from the SDSS Early Data Release (EDR). The locus we derive by eye (from objects with less than 0.1 mag error among 30,000 high-latitude objects) in the SDSS EDR corresponds to one line primarily following the SDSS halo/thick disk locus (Chen et al. 2001; Yasuda et al. 2001)

$$
\left(r^{*}-i^{*}\right) \sim 0.4029\left(g^{*}-r^{*}\right)-0.007,
$$

and another following the thin disk locus

$$
\left(r^{*}-i^{*}\right) \sim 14.286\left(g^{*}-r^{*}\right)-18.5 .
$$

An example of these color loci is shown in Figure 10, to be discussed in more detail in $\S 7.3$. By studying a variety of SDSS fields at seven different Galactic latitudes $(b=30,35$, $45,60,-45,-55,-65)$, we find variations of about $0.2 \mathrm{mag}$ in $\left(r^{\prime}-i^{\prime}\right)$ occur in the thin disk locus.

We also compare our magnitudes in $g^{*}$ and $r^{*}$ to magnitudes derived from the GSC2.2. We have derived the transformation between SDSS EDR and GSC2.2 magnitudes by direct comparison of the two surveys, using only stars between 17 th and 19th magnitude. After $3 \sigma$ clipping, the typical residuals are $0.2 \mathrm{mag}$, with zero points differing by $\sim 1 \%$ :

$$
\begin{aligned}
& g^{*}=1.032 J+0.1255(J-F)-0.851, \\
& r^{*}=0.982 F+0.1518(J-F)+0.451, \\
& i^{*}=0.994 F-0.1235(J-F)+0.281 .
\end{aligned}
$$

We note that most optical point sources brighter than 18th mag suffer from saturation effects in our NOAO $4 \mathrm{~m}$ imaging. While these objects constitute a small fraction $(\sim 6 \%)$ of our optically identified X-ray sources, they are a larger fraction $(\sim 10 \%)$ of our spectroscopically classified objects. We expect a full release of the SDSS will substantially alleviate this problem, as will our ongoing imaging study of these fields

\begin{tabular}{|c|c|c|c|c|c|c|}
\hline Fit Variable & $\begin{array}{c}e \\
(\mathrm{mag})\end{array}$ & $z$ & $k_{0}$ & $k_{1}$ & Number of Stars & $\begin{array}{c}\text { Fit rms } \\
(\mathrm{mag})\end{array}$ \\
\hline \multicolumn{7}{|c|}{ KPNO 4 m 2000 June 12} \\
\hline$g^{* \mathrm{a}} \ldots \ldots \ldots \ldots$ & 0.060 & 25.314 & 0.284 & -0.217 & 52 & 0.057 \\
\hline$r^{* \mathrm{~b}} \ldots \ldots \ldots \ldots$ & -0.055 & 25.424 & 0.207 & -0.155 & 49 & 0.068 \\
\hline$i^{* \mathrm{~b}} \ldots \ldots \ldots \ldots$ & -0.185 & 25.194 & 0.191 & -0.117 & 54 & 0.250 \\
\hline$g^{*}-r^{*} \ldots \ldots \ldots$ & 1.021 & -0.087 & 0.044 & -0.036 & 54 & 0.111 \\
\hline$r^{*}-i^{*} \ldots \ldots \ldots$ & 0.978 & 0.362 & 0.064 & -0.050 & 47 & 0.031 \\
\hline \multicolumn{7}{|c|}{ CTIO 4 m 2000 September 28} \\
\hline$g^{* \mathrm{a}} \ldots \ldots \ldots \ldots$ & 0.038 & 25.695 & 0.257 & -0.210 & 43 & 0.027 \\
\hline$r^{* \mathrm{~b}} \ldots \ldots \ldots \ldots$ & -0.020 & 25.794 & 0.145 & -0.118 & 43 & 0.033 \\
\hline$i^{* \mathrm{~b}} \ldots \ldots \ldots \ldots$ & 0.083 & 25.358 & 0.089 & -0.072 & 41 & 0.041 \\
\hline$g^{*}-r^{*} \ldots \ldots \ldots$ & 1.011 & -0.087 & 0.110 & -0.090 & 41 & 0.037 \\
\hline$r^{*}-i^{*} \ldots \ldots \ldots$ & 0.903 & 0.410 & 0.078 & -0.064 & 43 & 0.027 \\
\hline
\end{tabular}
with the SAO FLWO $1.2 \mathrm{~m}$ and 4shooter CCD on Mount Hopkins.

Since most of our X-ray sources are extragalactic, before calculations of optical luminosities, we correct the optical magnitude measurements for Galactic extinction using the

TABLE 3

Nightly Photometric Calibration Coefficients

a Uses $\left(g^{*}-r^{*}\right)$ colors in fit.

b Uses $\left(r^{*}-i^{*}\right)$ colors in fit. 
maps of Schlegel, Finkbeiner, \& Davis (1998). We assume an $R_{V}=3.1$ absorbing medium, and absorption in the SDSS bands as given by Schneider et al. (2002).

\section{X-RAY TO OPTICAL SOURCE MATCHING}

For the ChaMP, Kim et al. (2003a) have carried out extensive simulations of point sources generated using the SAOSAC raytrace program ${ }^{31}$ and detected using CIAO/wavdetect (Freeman et al. 2002). For weak sources of $\sim 20$ counts between $8^{\prime}$ and $10^{\prime}$ off-axis from the aim point, the reported X-ray centroid position is correct within 1 "'. 8 , corresponding to a $1 \sigma$ confidence contour.

The source naming convention of the ChaMP as officially registered with the IAU is given with a prefix CXOMP (Chandra X-ray Observatory Multiwavelength Project) and affixed with the truncated J2000 position of the X-ray source (CXOMP Jhhmmss.s \pm ddmmss) after a mean field offset correction is applied, derived from the positional matching of optical and X-ray sources in each field.

\subsection{Matching Criteria}

\subsubsection{Automated Matching}

We obtain candidate optical identifications of X-ray sources in each field using a two-stage automated position matching process between optical and X-ray source positions. In the first stage, the subset of X-ray sources within $5^{\prime}$ of the Chandra aim point and with X-ray $B(0.3-8 \mathrm{keV})$ detections of more than 20 counts are matched to optical sources from our CCD imaging, using position matching with a radial offset matching tolerance, in arcsec, $R_{\text {match }}=\theta / \log _{10}(B)$, where $\theta$ is the offaxis angle in arcminutes, and $B$ is the X-ray source counts, but $R_{\text {match }}$ is limited to a minimum of $3^{\prime \prime}$ for all sources. This matching tolerance encompasses the wavdetect centroid positional uncertainty variation with off-axis angle and source counts, using the refined source position estimator described in Kim et al. (2003a). The optical position errors are negligible in comparison. In those cases where there are multiple optical matches, the closest match is used. The median X-ray to optical position translational offset of these matched sources is then applied to all the X-ray sources for the field, to bring the optical and X-ray source lists onto the same coordinate frame. The X-ray position corrections are typically less than $1^{\prime \prime}$, which is consistent with the specified absolute position accuracy $^{32}$ for the Chandra observatory and wavdetect source positions. Figure 3 shows optical matches overlaid directly on the ACIS-I image of Obs. ID 536.

In the second stage of position matching, optical matching is performed for all X-ray sources with X-ray $B$ detections of more than 5 counts, using the same matching tolerance $R_{\text {match }}$ as above. Multiple optical matches for an X-ray source are allowed in this stage and are passed to the visual inspection process described next. Extended X-ray source centroids may have larger position errors than are predicted by our point source formula. Furthermore, in some cases no corresponding optical cluster galaxy may exist close to the cluster X-ray centroid. If no optical counterpart is found in the automated matching routine for these or other cases, visual inspection provides a second chance to identify counterparts.

\footnotetext{
${ }^{31}$ See http://hea-www.harvard.edu/MST.

${ }^{32} \mathrm{See} \mathrm{http}: / /$ asc.harvard.edu/cal/ASPECT for a detailed discussion of Chandra aspect solutions.
}

\subsubsection{Visual Inspection}

After our automated $\mathrm{O} / \mathrm{X}$ matching between detected sources from XPIPE and SExtractor source positions has been performed, we examine the images by eye. An example of the plots used for visual inspection is shown in Figure 4. In many cases, no obvious optical counterpart can be detected, while in other cases there are several (source confusion). Even with typical astrometric accuracy of an arcsec, at these faint optical magnitudes and X-ray fluxes we frequently find several optical counterpart candidates. Beyond $r^{*} \sim 20$, galaxies dominate the optical number counts, and by 24th magnitude, cumulative source counts are near $10^{5} \mathrm{deg}^{-2}$ (Kümmel \& Wagner 2001). At these faint magnitudes, the probability of finding an unrelated optical source by chance within any $3^{\prime \prime}$ circle becomes $\sim 20 \%$. The small PSF and accurate centroiding of Chandra become crucial at these faint magnitudes, as does careful source inspection.

\subsection{Fraction with Optical Counterparts}

During visual inspection of the optical field of each X-ray source, we assign a match confidence to each plausible counterpart. To consider a sample as uncontaminated as possible by incorrect matches, we have removed from further optical analysis below $45 \mathrm{X}$-ray sources with poor or ambiguous counterpart assignments.

The resulting fraction of X-ray sources with confidently matched optical counterparts is shown in Table 4. We have not yet achieved the desired optical magnitude limits (set to match optical counterparts for $90 \%$ of ROSAT AGNs) in all these deep Chandra fields, but the imaging has served to facilitate spectroscopy to $r^{*} \sim 21$ and to provide fluxes and colors to still deeper limits. Lower achieved match fractions also reflect the inclusion by Chandra of new $f_{\mathrm{X}} / f_{\text {opt }}$ populations atypical for ROSAT, such as heavily absorbed AGNs.

Excluding the $\sim 10 \%$ of sources with more than $200 \mathrm{X}$-ray counts, the mean/median X-ray flux for matched sources (3.5/2.4 in units of $10^{-15} \mathrm{ergs} \mathrm{cm}^{-2} \mathrm{~s}^{-1}$ ) exceeds the flux for unmatched sources $(2.6 / 1.6)$ as expected, but not strongly. The mean off-axis angle of unmatched sources $\left(\theta=6 .^{\prime} 7 \pm 3 .^{\prime} 9\right)$ is indistinguishable from that of matched sources $(6.2 \pm 3.2$; both quoted errors are $1 \sigma \mathrm{rms}$ ).

For a typical ChaMP source in these fields, the median X-ray $(0.5-2 \mathrm{keV})$ flux is $2.4 \times 10^{-15} \mathrm{ergs} \mathrm{cm}^{-2} \mathrm{~s}^{-1}$, where according to the $\log N-\log S$, the cumulative $(0.5-2 \mathrm{keV}) \mathrm{X}$-ray source density attains about $30 \mathrm{deg}^{-2}$ (e.g., Rosati et al. 2002). The probability of two X-ray sources falling within $10^{\prime \prime}$ of each other by chance is less than 0.001 . On the other hand, the number of true multiple sources is of great interest; they may either be lensed images or perhaps binary AGNs, possibly interactiontriggered (e.g., Green et al. 2002). Since these latter types are expected to be rare, a large-area survey like the ChaMP is needed to find them. New close pairs of X-ray sources with optical counterparts are described in the Appendix.

\section{OPTICAL SPECTROSCOPY}

The nature of many individual sources, especially those with unusual properties, cannot be reliably determined until spectroscopy is obtained. Our spectroscopy targets all objects in a representative subset of 40 ChaMP cycle $1+2$ fields, yielding $\sim 1000$ objects for which we will obtain the most detailed information. With our spectroscopic subsample, we will classify sources by type, determine redshifts and hence 


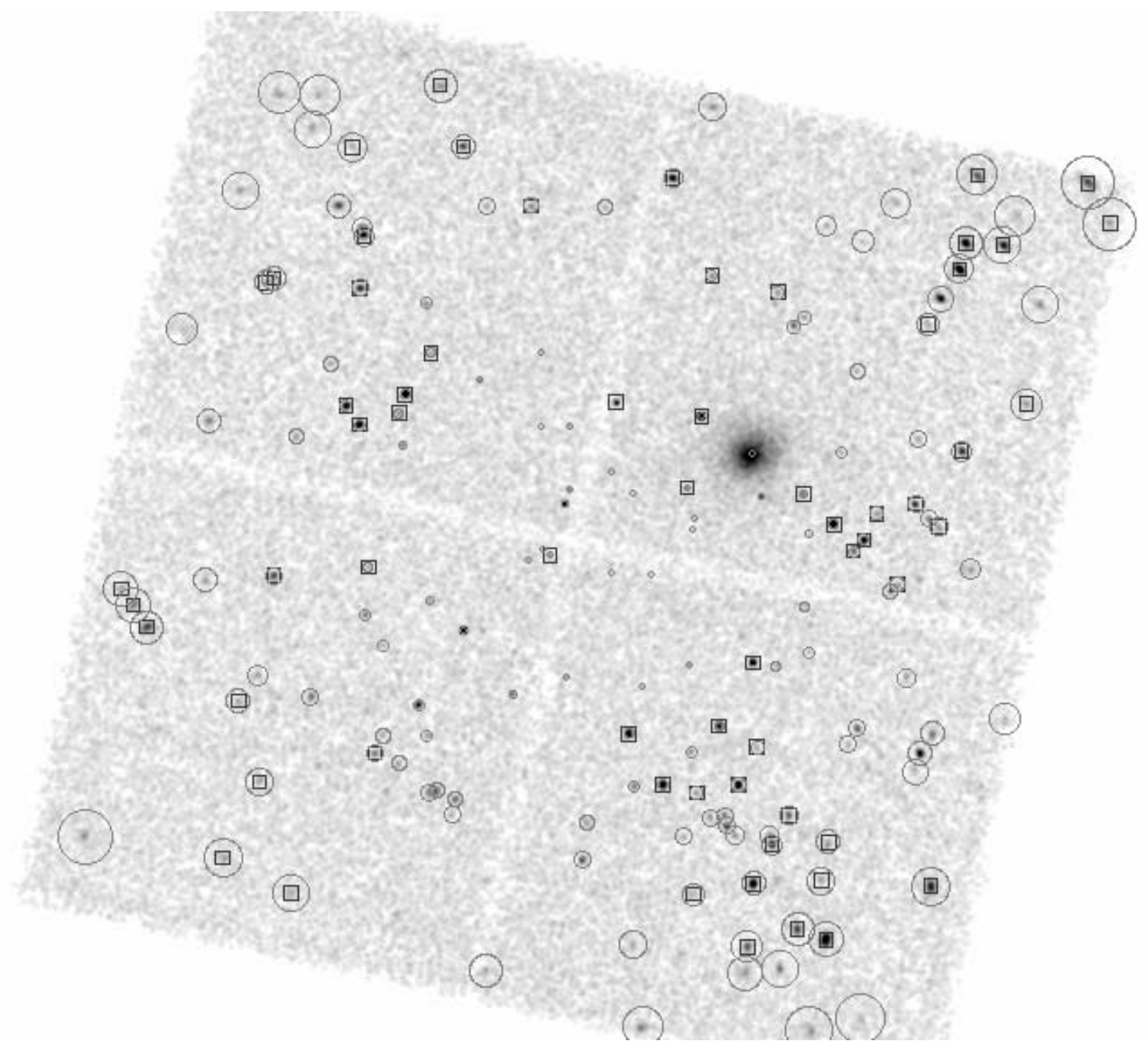

FIG. 3. - Chandra image of the field of Obs. ID 536 (target MS 1137.5+6625). The $16^{\prime} \times 16^{\prime}$ ACIS-I $(0.3-5 \mathrm{keV})$ image (north is up, east is to the left as in all images) was smoothed with a 3 pixel (1".5) Gaussian for clarity. The 156 X-ray sources detected to $2 \times 10^{-16} \mathrm{ergs}^{-2} \mathrm{~cm}^{-1}$ using the ChaMP's X-ray pipeline are marked with circles sized to the Chandra PSF $(90 \%$ EE). To the $125 \mathrm{~S} / \mathrm{N}>2$ X-ray sources detected in these four chips, we have matched 80 optical sources detected in our combined $4 \mathrm{~m} /$ Mosaic images to $r^{*} \sim 25$, indicated by $15^{\prime \prime}$ boxes. The target cluster is easily visible as the extended source NW of center and is detected as a single source by XPIPE, but like all target objects it is excluded from ChaMP analysis. [See the electronic edition of the Journal for a color version of this figure.]

luminosities and look-back times for evolutionary studies, and test photometric determinations of redshift and classification.

The mean magnitude of optical counterparts is $r^{\prime} \sim 22$ for a 23 ks high Galactic latitude Chandra exposure, and so fields of this Chandra exposure or larger typically have at least 15 sources suitable for fiber spectroscopy. Multifiber spectroscopy, even on 4 to $6 \mathrm{~m}$ telescopes, allows us to consistently obtain source classification and redshifts to $r^{\prime} \sim 20$, and up to 1.5 mag fainter for objects with strong emission lines. Our overall spectroscopic observing strategy has been to use single-slit (FLWO $1.5 \mathrm{~m}$ with FAST) spectroscopy for isolated $r^{\prime}<17$ sources in shallow Chandra fields, and in our deeper fields, which might otherwise introduce scattered light problems for multiobject spectroscopy. Multifiber wide-field spectroscopy with the HYDRA spectrographs (Barden et al. 1994) on the WIYN $3.5 \mathrm{~m}$ and CTIO $4 \mathrm{~m}$ telescopes enables us to acquire spectra for sources with $17<r^{\prime}<21$. A subset of faint ChaMP sources $\left(21<r^{\prime}<23\right)$ in fields designated for spectroscopic follow-up are being observed with $6 \mathrm{~m}$ class telescopes such as Magellan and the MMT. Table 5 lists the technical specifications and configurations for each spectroscopic facility used for results presented herein.
Of the six fields studied here, each has been observed with a multifiber spectrograph on either the WIYN or CTIO $4 \mathrm{~m}$ through $2^{\prime \prime}$ fibers (Table 6). An average of 15 spectra per field are acquired with this configuration. We obtained multislit observations for 17 sources with Keck-I/LRIS or Magellan/LDSS2. Single-slit observations of 19 sources were acquired with the FLWO $1.5 \mathrm{~m}$ when optically bright $\left(r^{*}<17\right)$ and MMT or Magellan when faint. We perform standard optical spectroscopic reductions within the IRAF environment. For multifiber reductions, we use the IRAF task dohydra. To optimize sky subtraction, we cross-correlate all dispersion-corrected (wavelength calibrated) sky spectra from random sky locations in the field against the object+sky spectrum from each object fiber using the task xcsao within the external IRAF package rvsao. We then median-combine the nine sky fibers whose spectra correlate most closely with the total object+sky spectrum. We find this method to be superior to removal of a simple field-averaged sky spectrum for faint objects. Slit spectra were reduced using the IRAF task apall with a background signal measured locally for each object within the same slit. The multifiber spectra are only nominally fluxcalibrated, since we use just one or two standards per night 

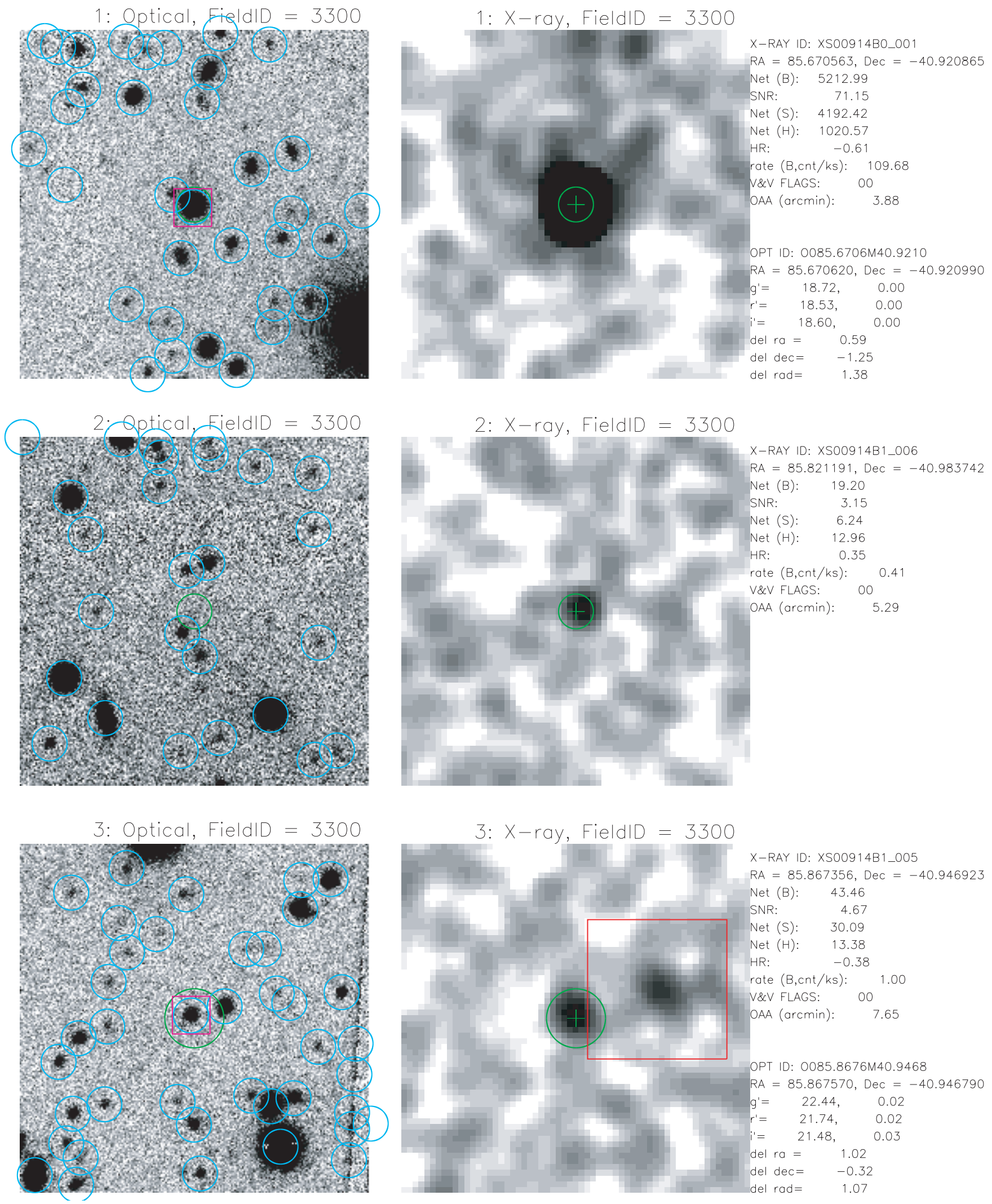

FIG. 4.-Example from Obs. ID 914 (target CL J0542.8-4100) of the pages used for initial visual inspection of X-ray source matches. Right panels: A 1' field centered on a detected X-ray source is shown, with north up and east to the left. The $0.3-8 \mathrm{keV} \mathrm{X-ray} \mathrm{image} \mathrm{is} \mathrm{smoothed} \mathrm{with} \mathrm{a} 2$ pixel (1") Gaussian for clarity, which facilitates source inspection and examination of background and confusion effects. Green circles around the X-ray source have size equal to the PSF (50\% EE) at the source position. Crosses show the wavdetect X-ray centroid. If other X-ray sources are detected within the field, they are marked by a $20^{\prime \prime}$ box. Left panels: The corresponding optical $r^{*}$ images shows all optical sources detected by SExtractor, marked with $3^{\prime \prime}$ circles. The Chandra 50\% EE circle is reproduced at the X-ray source position. When our automated matching procedure finds an optical counterpart, the optical source is marked with a $3^{\prime \prime}$ box. X-ray and optical photometric data are displayed to the far right. 
TABLE 4

Source Matching

\begin{tabular}{|c|c|c|c|c|c|}
\hline Obs. ID & $N_{\mathrm{X}}{ }^{\mathrm{a}}$ & $N_{\mathrm{XO}}{ }^{\mathrm{b}}$ & $\begin{array}{c}f_{\mathrm{XO}}{ }^{\mathrm{b}} \\
(\%)\end{array}$ & $N_{\mathrm{XOS}}{ }^{\mathrm{c}}$ & $\begin{array}{r}f_{\mathrm{XOS}}{ }^{\mathrm{d}} \\
(\%)\end{array}$ \\
\hline $536 \ldots \ldots \ldots \ldots \ldots \ldots$ & 140 & 77 & 55 & 33 & 70 \\
\hline 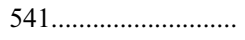 & 65 & 45 & 69 & 24 & 72 \\
\hline 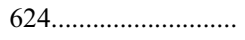 & 58 & 45 & 78 & 19 & 74 \\
\hline 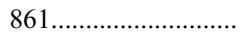 & 80 & 57 & 71 & 14 & 59 \\
\hline 914 & 88 & 65 & 74 & 20 & 50 \\
\hline $928 \ldots \ldots \ldots \ldots \ldots \ldots \ldots . .$. & 55 & 42 & 76 & 15 & 50 \\
\hline Total ............................. & 486 & 331 & 71 & 125 & 63 \\
\hline
\end{tabular}

${ }^{a}$ Number of X-ray sources detected in each Chandra image and also having $\mathbf{S} / \mathbf{N}>2$ in counts as described in $\S 3$.

b Number $N$ or percent $f$ of $\mathrm{X}$-ray sources with high confidence optical counterparts as described in $\S 5$.

c Number $N$ of optical counterparts with spectroscopic classification.

d Percent $f$ of $r^{*} \leq 22$ optical counterparts with spectroscopic classification.

down a single central fiber. One or two standards are used for the single-slit spectra.

\subsection{Object Types and Redshifts}

We classify objects spectroscopically simply as broad-line AGN (BLAGN; FWHM $>1000 \mathrm{~km} \mathrm{~s}^{-1}$ ) narrow emission line galaxy (NELG; FWHM $<1000 \mathrm{~km} \mathrm{~s}^{-1}$; some of which may be AGN), absorption line galaxy (ALG), or star. Objects with high $\mathrm{S} / \mathrm{N}(>20)$ and no identifying features are denoted as BL Lac candidates, as are those whose identified Ca II $4000 \AA$ region shows a break contrast of less than 0.25 (see $\S 7.4 .3$ ). Our classification confidence level is denoted as well $(0$ for not classifiable, 1 insecure, 2 secure). These are all strictly optical classifications.

Redshifts are measured for extragalactic objects using the radial velocity package $r v s a o$ under the IRAF environment. This method depends on the optical classification. Quasar spectra are quite similar over a broad range of redshifts and luminosities (Forster et al. 2001), so that - absent the effects of broad absorption lines (BALs) or a strong Ly $\alpha$ forest absorption - a single high $\mathrm{S} / \mathrm{N}$ composite serves well as a redshift template. For BLAGNs, we use the quasar template created from over 2200 quasar spectra collected by the SDSS (Berk et al. 2001).
We run xcsao over the full redshift range in 0.1 redshift intervals, choosing the strongest correlation as the true redshift. Since the correlation strength depends strongly on $\mathrm{S} / \mathrm{N}$ and artifacts such as poorly subtracted sky lines, we inspect and visually verify all redshifts and classifications by comparison with overplotted templates. For ALG we use xcsao with a synthetic absorption line template. Whenever narrow emission lines are present, we elect to measure the redshift from these features to reduce the associated error. For NELG, the task emsao is used to identify emission lines using a line list. For stars, we assume an effective radial velocity of zero and allow xcsao to correlate against stellar template spectra from Jacoby, Hunter, \& Christian (1984). Our comparisons between by-eye classifications and these automated results suggest an uncertainty of about one full spectral type.

For X-ray sources with $r^{*}<22$ optical counterparts, we have already achieved $54 \%$ completeness. All sources with $r>21$ that we have identified spectroscopically to date are BLAGNs or NELG, because their strong emission lines facilitate classification and redshift determination. We are currently extending to $90 \%$ completeness at $r^{*}=21$ in at least 20 ChaMP fields specifically to map the space density of X-S AGNs.

TABLE 5

Spectroscopic Facilities

\begin{tabular}{|c|c|c|c|c|c|c|}
\hline Telescope & Instrument & Mode & Grating/Grism & $\begin{array}{c}\lambda \text { Range } \\
(\AA)\end{array}$ & $\begin{array}{c}R \\
(\lambda / \Delta \lambda)\end{array}$ & $\begin{array}{c}\text { Spectral } \\
\text { Resolution } \\
(\AA)\end{array}$ \\
\hline 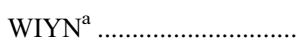 & HYDRA/RBS & Multifiber & 316 at 7.0 & $4500-9000$ & 950 & 7.8 \\
\hline CTIO Blanco 4 m............ & HYDRA & Multifiber & KPGL3 & $4600-7400$ & 1300 & 4.6 \\
\hline 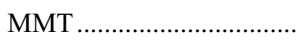 & Blue Channel & Single slit & 300 line $\mathrm{mm}^{-1}$ & $3500-8300$ & 800 & 8.8 \\
\hline 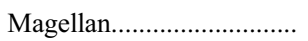 & Boller \& Chivens & Single slit & 1200 line $\mathrm{mm}^{-1}$ & $4200-5800$ & 2083 & 2.4 \\
\hline 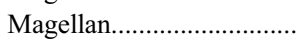 & LDSS-2 & Single slit & $\mathrm{med} / \mathrm{red}$ & $4000-8500^{\mathrm{b}}$ & 520 & 13.5 \\
\hline $\mathrm{Keck}^{\mathrm{c}}$ & LRIS $^{\mathrm{d}}$ & Multislit & 300 line $\mathrm{mm}^{-1}$ & $4000-9000^{\mathrm{b}}$ & & \\
\hline FLWO $1.5 \mathrm{~m}$ & FAST & Single slit & 300 line $\mathrm{mm}^{-1}$ & $3600-7500$ & 850 & 5.9 \\
\hline
\end{tabular}

a The WIYN Observatory is a joint facility of the University of Wisconsin Madison, Indiana University, Yale University, and the National Optical Astronomy Observatory.

${ }^{b}$ Spectral coverage can vary as a function of slit position in the mask.

${ }^{c}$ The W. M. Keck Observatory is operated as a scientific partnership among the California Institute of Technology, the University of California, and the National Aeronautics and Space Administration, and was made possible by the generous financial support of the W. M. Keck Foundation.

${ }^{\mathrm{d}}$ LRIS; Oke et al. 1995. 
TABLE 6

Optical Spectroscopy

\begin{tabular}{|c|c|c|c|c|}
\hline Obs. ID & Telescope & Instrument & UT Date & Number of Spectra \\
\hline \multirow[t]{4}{*}{$536 \ldots \ldots \ldots \ldots \ldots \ldots \ldots \ldots$} & Keck & LRIS & 2000 May 15 & 5 \\
\hline & WIYN & HYDRA & 2001 Apr 07 & 7 \\
\hline & WIYN & HYDRA & 2003 Jan 30 & 13 \\
\hline & WIYN & HYDRA & 2003 Jan 31 & 4 \\
\hline \multirow[t]{4}{*}{$541 \ldots \ldots \ldots \ldots \ldots \ldots \ldots \ldots \ldots$} & WIYN & HYDRA & 2001 Apr 02 & 12 \\
\hline & WIYN & HYDRA & 2001 Apr 07 & 9 \\
\hline & MMT & Blue channel & 2001 May 26 & 1 \\
\hline & MMT & Blue channel & 2002 Jul 12 & 2 \\
\hline \multirow[t]{2}{*}{$624 \ldots \ldots \ldots \ldots \ldots \ldots \ldots$} & CTIO 4 m & HYDRA & 2001 Oct 16 & 11 \\
\hline & Magellan & LDSS-2 & 2002 Dec 03 & 5 \\
\hline \multirow[t]{4}{*}{$861 \ldots \ldots \ldots \ldots \ldots \ldots \ldots \ldots}$. & WIYN & HYDRA & 2001 Sep 19 & 10 \\
\hline & WIYN & HYDRA & 2001 Sep 20 & 2 \\
\hline & MMT & Blue channel & 2002 Jul 11 & 1 \\
\hline & FLWO $1.5 \mathrm{~m}$ & FAST & 2001 Oct 15 & 2 \\
\hline \multirow[t]{2}{*}{ 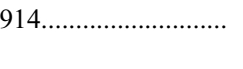 } & CTIO $4 \mathrm{~m}$ & HYDRA & 2001 Oct 17 & 15 \\
\hline & Magellan & LDSS-2 & 2002 Dec 01 & 5 \\
\hline \multirow[t]{4}{*}{928} & CTIO $4 \mathrm{~m}$ & HYDRA & 2001 Oct 15 & 7 \\
\hline & Magellan & LDSS-2 & 2001 May 21 & 1 \\
\hline & Magellan & $\mathrm{BSC}$ & $2002 \mathrm{Jul} 15$ & 5 \\
\hline & Magellan & $\mathrm{BSC}$ & $2002 \mathrm{Jul} 15$ & 1 \\
\hline
\end{tabular}

\section{RESULTS}

\subsection{Fluxes, Redshifts, and Object Types}

Figure 5 shows a plot of the $r^{*}$ band magnitude against $\log f_{\mathrm{X}}$ calculated in the $0.5-2 \mathrm{keV}$ band. Overplotted are three lines of constant $f_{\mathrm{X}} / f_{r}$ at 10,1 , and 0.1 . We calculate this $\mathrm{X}$-ray to optical flux ratio similarly to Hornschemeier et al. (2001) or Manners et al. (2003), using our SDSS $r^{*}$ magnitudes:

$$
\log \frac{f_{\mathrm{X}}}{f_{r}}=\log f_{\mathrm{X}}+5.67+0.4 r^{*},
$$

and use this as our measure of $f_{\mathrm{X}} / f_{\mathrm{opt}}$ throughout. For comparison, we also plot a line showing the typical relation expected for O-S quasars (Green et al. 1995), assuming a constant $\alpha_{o x}=1.5$, which corresponds to $\log \left(f_{\mathrm{X}} / f_{r}\right)=-0.57$. ChaMP quasars clearly differ from O-S quasars in the expected sense-X-S objects have typically stronger X-ray emission relative to optical [ $\left.\left\langle\log \left(f_{\mathrm{X}} / f_{r}\right)\right\rangle=-0.15\right]$. Alternatively, even optically "dull" quasars are still detected by the ChaMP, since luminous X-ray emission is the primary signature of AGN activity. BLAGNs are X-ray bright, being largely limited to $f_{\mathrm{X}} / f_{r}>0.1$. Galaxies tend to appear toward fainter X-ray fluxes but span a wide range of optical brightness. Stars are essentially limited to $f_{\mathrm{X}} / f_{r}<0.1$ and $r^{*}<20$, which corresponds to a typical $\mathrm{M}$ dwarf at distances of $400-1000 \mathrm{pc}$.

Apparent trends between fluxes or colors in any survey can be due to a combination of survey sensitivity limits. To investigate selection effects in a first approximation, we have generated a very deep simulated SDSS quasar catalog as described in Fan (1999), based on their observed optical luminosity function and its evolution, and including the effects of emission lines and intervening absorption. We have extended the simulation to include AGNs with $M_{B}<-21$ out to a redshift of 6 , corresponding to $z^{\prime}$ magnitudes to 28 . We then assume that the mean observed ratio $\log \left(f_{\mathrm{X}} / f_{r}\right)$ has a constant value of -0.14 with a dispersion $\sigma_{x r}=0.4$. (These are the actual values we find for all 41 quasars in our sample in the flux regime $f_{\mathrm{X}}>5 \times 10^{-15} \mathrm{ergs} \mathrm{cm}^{-2} \mathrm{~s}^{-1}$, where our optical matching rate is high.) From the $r^{\prime}$ mag for each simulated quasar, we then generate X-ray fluxes, luminosities, etc. Finally, we can cut the sample at any desired mag or X-ray flux. Primarily, we use a cut of $r^{\prime}<25$ and $\log f_{\mathrm{X}}=-15$, similar to our actual final sample limits. For most figures which require redshifts, we use a cut of $r^{\prime}<22$ more appropriate to the spectroscopic sample. The simulated sample is thus based on a quasar OLF with an assumed constant observed $f_{\mathrm{X}} / f_{r}$ for an X-S sample, rather than on the actual quasar XLF. Indeed, a prime goal of the ChaMP is to measure the XLF of X-S quasars with improved statistics at fainter fluxes where it is not yet well characterized and to compare the XLF and OLF particularly at redshifts beyond 2.

In Figure 5 , we plot the mean $r^{\prime}$ versus $\log f_{\mathrm{X}}$-values of quasars in this simulation subsample, derived in redshift bins of $\Delta z=0.5$. The track matches well with the overall trend of BLAGNs. There is large dispersion in both optical and X-ray fluxes about this mean track. Substantial incompleteness in optical IDs sets in fainter than $r^{*} \sim 23.5$, where a decreasing fraction of X-ray sources have optical counterparts. However, as discussed below in $\S 7.2$, the characteristics of X-S quasars clearly differ from the O-S quasars on which the OLF is based.

The proportional representation of different object types changes strongly with redshift. Figure 6 shows the overall redshift distribution of objects classified in our spectroscopy. BLAGNs are from $z \sim 0.2$ to beyond $z \sim 3$. We can detect and classify quasars to $r^{*}$ magnitudes considerably fainter than galaxies due to their strong broad emission lines. We only see galaxies (both NELGs and ALGs) to $z \sim 0.8$. This effect is due primarily to magnitude limits; our spectroscopic limit so far is $r^{*} \sim 21$ for objects with no strong emission lines within the covered optical wavelength range. Figure 7 highlights this by showing that the faintest classified ALGs are at $r^{*}=21$.

From galaxies in the Hubble Deep Field with good completeness to $R=23$ (Cohen et al. 2000), the median redshift of galaxies at $R=21$ is about 0.5 . By contrast, our 


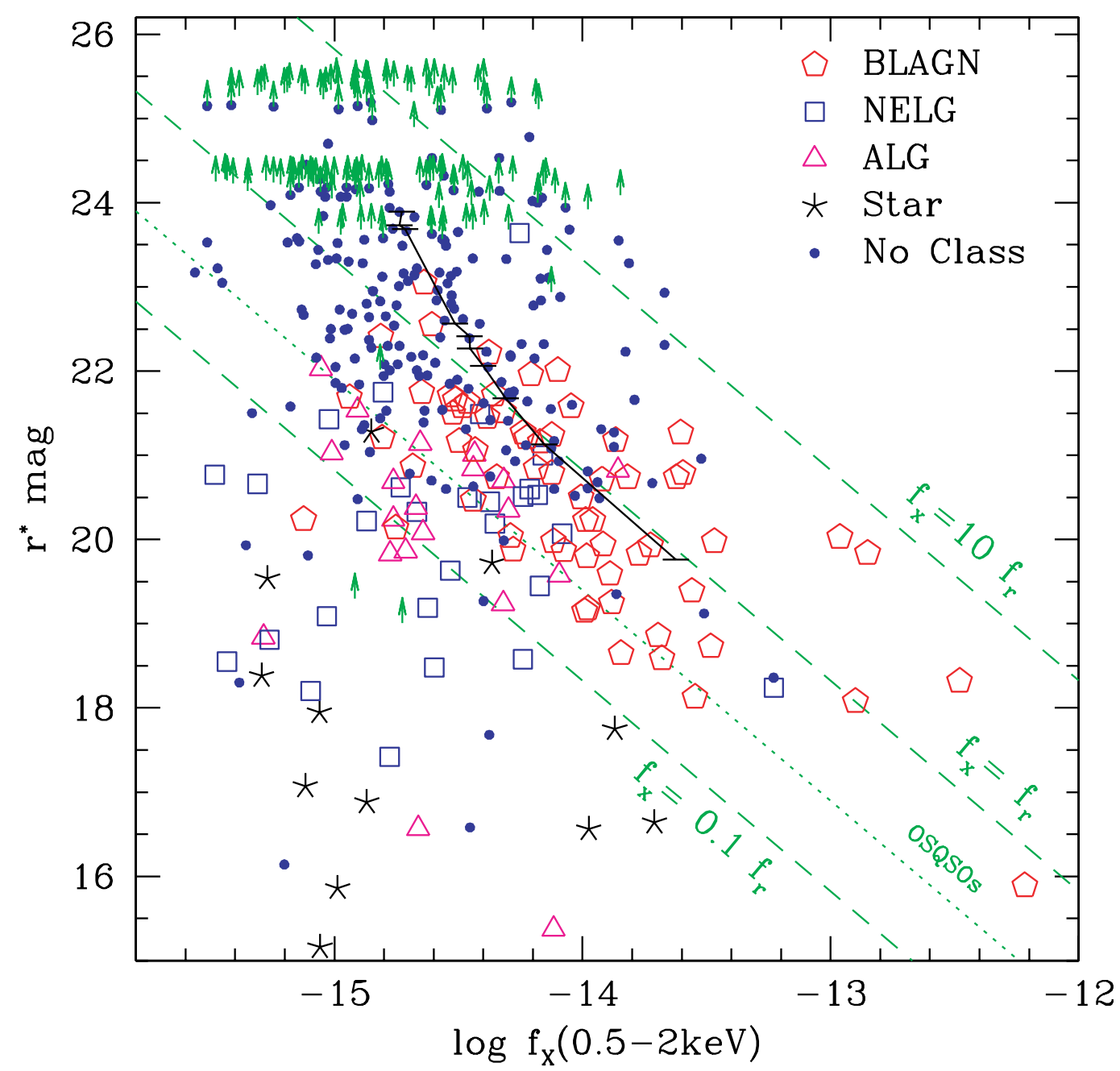

FIG. 5. - SDSS $r^{*}$ band magnitudes vs. Chandra $\log f_{\mathrm{X}}$ (calculated for the $0.5-2 \mathrm{keV}$ band from the broadband $0.3-8 \mathrm{keV}$ counts) for all X-ray sources in the six fields studied here. Overplotted are three dashed lines of constant $f_{\mathrm{X}} / f_{r}$ at $0.1,1$, and 10 calculated as described in $\S 7.1$. A fourth $($ dotted) line marked OSQSOs shows the typical mean values for optically selected quasars $\left[\log \left(f_{\mathrm{X}} / f_{r}\right)=-0.57\right]$. Large symbols for different optical source types are shown as determined from our spectroscopy: broad-line AGNs (BLAGNs), narrow emission line galaxies (NELG), absorption line galaxies (ALG), and stars. Any X-ray source not detected in our $r^{*}$-band images is marked with an arrow at the position of its $r^{*}$ magnitude limit. Small filled circles indicate sources with an optical counterpart in at least one filter. The curve traces the mean expected quasar track for $z=0.5$ redshift bins for the simulation described in $\S 7$, imposed flux limits of $\log f_{\mathrm{X}}=-15$ and $r^{*}=25$. The bright end point has $\bar{z}=0.37$, while the faint end has $\bar{z}=4.6$. Many of the optically faint objects are likely to be high-z AGNs. Magnitudes of point sources brighter than 18 suffer from saturation effects.

median $r^{*}$ mag for $z=0.5$ galaxies is significantly brighter at that redshift. This may not be due to incompleteness of optical follow-up; given both the possibility of an AGN contribution and the known correlation between bulge luminosity and black hole mass, we note that X-S galaxies are likely to be atypically luminous in the optical band.

\subsection{Luminosities and X-Ray/Optical Ratio}

Figure 8 shows the $0.5-2 \mathrm{keV}$ X-ray luminosity of spectroscopically classified galaxies and AGNs as a function of redshift. BLAGNs span a higher range of luminosities than do galaxies, but with some overlap. Overplotted is the sensitivity of the ROSAT Deep Survey assuming their quoted limit $f_{\mathrm{X}}=5.5 \times 10^{-15}$; (Lehmann et al. 2001). The ChaMP samples luminosities 5-10 times fainter at a given redshift even assuming a more optimistic limit (from their Fig. 3) of $2 \times 10^{-15}$.

The slope of a hypothetical power law connecting $2500 \AA$ and $2 \mathrm{keV}$ is defined as $\alpha_{o x}=0.384 \log \left(L_{\mathrm{opt}} / L_{\mathrm{X}}\right)$ (where these are monochromatic luminosities at $2500 \AA$ and $2 \mathrm{keV}$ ), so that $\alpha_{o x}$ is larger for objects with stronger optical emission relative to X-ray. We note that our definition of $\alpha_{o x}$ here includes no mean $k$-corrections for emission lines, intervening or intrinsic absorbers, so $\alpha_{o x}$ is directly related to $\log \left(f_{\mathrm{X}} / f_{r}\right)$, which we prefer. $^{33}$

O-S AGN samples show typical $\alpha_{o x}$-values of about 1.5 (Green et al. 1995) corresponding to $\log \left(f_{\mathrm{X}} / f_{r}\right)=-0.57$. We find for the current sample of ChaMP BLAGNs a somewhat lower value of $\left\langle\alpha_{o x}\right\rangle=1.36 \pm 0.15\left[\log \left(f_{\mathrm{X}} / f_{r}\right)=-0.19\right]$. La Franca et al. (1995) found a similar mean $\alpha_{o x}$ of 1.3 for X-S AGNs $\left[\log \left(f_{\mathrm{X}} / f_{r}\right)=0\right]$. X-ray surveys select X-ray bright objects by definition, and so are complementary to O-S samples.

A significant correlation between optical luminosity and $\alpha_{o x}$ has been noted in many studies based on O-S quasars (Vignali

\footnotetext{
${ }^{33}$ For convenience, the exact conversion between the two is $\alpha_{o x}=$ $-0.379 \log \left(f_{\mathrm{X}} / f_{r}\right)+1.286$.
} 


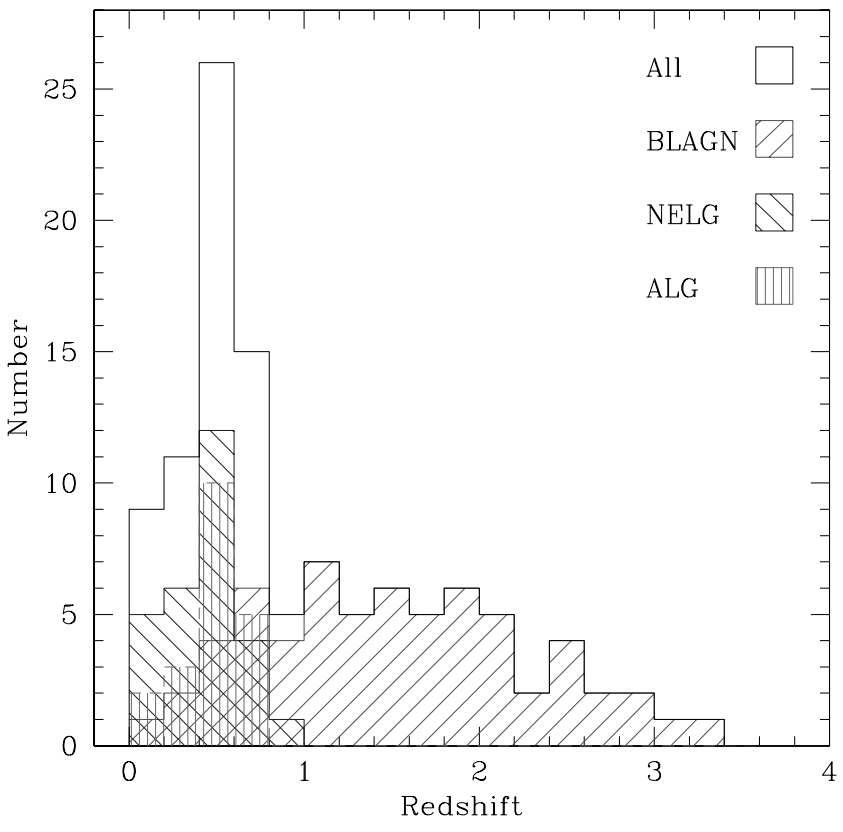

FIG. 6.-Redshift histogram of classified extragalactic sources in these six fields. Most objects showing no broad-line component are below $z=0.8$, likely caused by our typical spectroscopic magnitude limit near 21. [See the electronic edition of the Journal for a color version of this figure.]

et al. 2001, 2003; Yuan et al. 1998; Green et al. 1995; Wilkes et al. 1994). One cause might be low-energy X-ray absorption as reported in quasars at higher redshifts (Vignali et al. 2001; Yuan et al. 2000; Fiore et al. 1998; Elvis et al. 1994). Hypothesized to be due to the presence of large gas quantities in primeval galaxies, such absorption might also be related to

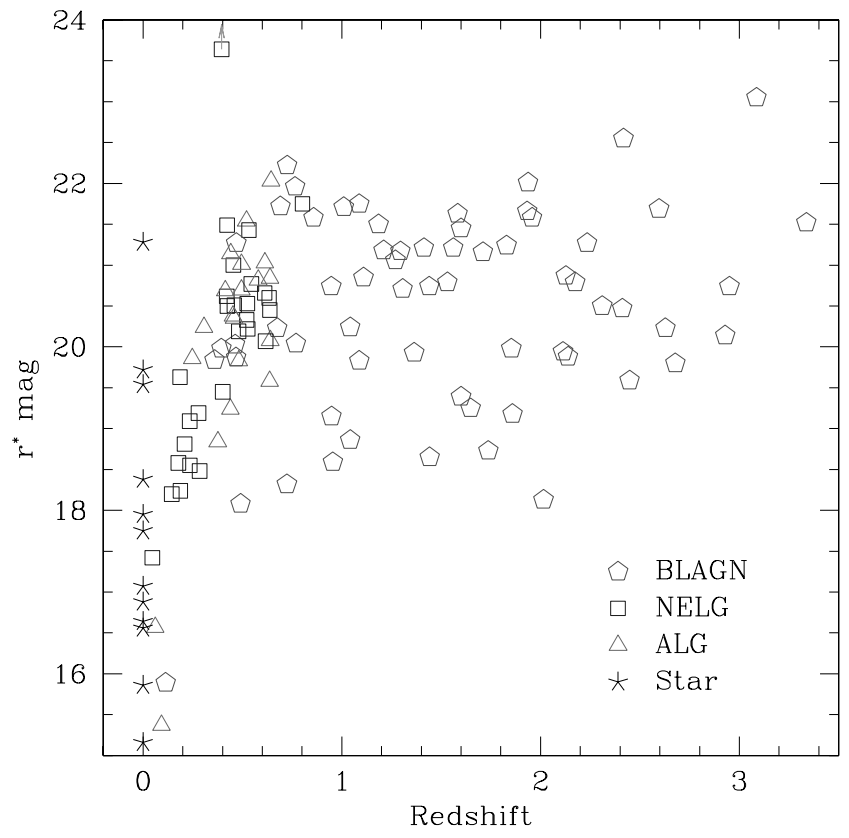

FIG. 7.- SDSS $r^{*}$ band magnitudes vs. redshift. Galaxies of all types are seen out to $z \sim 0.8$, beyond which they are typically too faint for spectroscopic classification. BLAGNs can be detected at all redshifts and extend well beyond our nominal $r^{*}=21$ spectroscopic limit due to their strong broad emission lines. The wide range of $r^{*}$ magnitudes at each redshift reflects both the breadth of object types detected, and the range of exposure time across and between fields. [See the electronic edition of the Journal for a color version of this figure.]

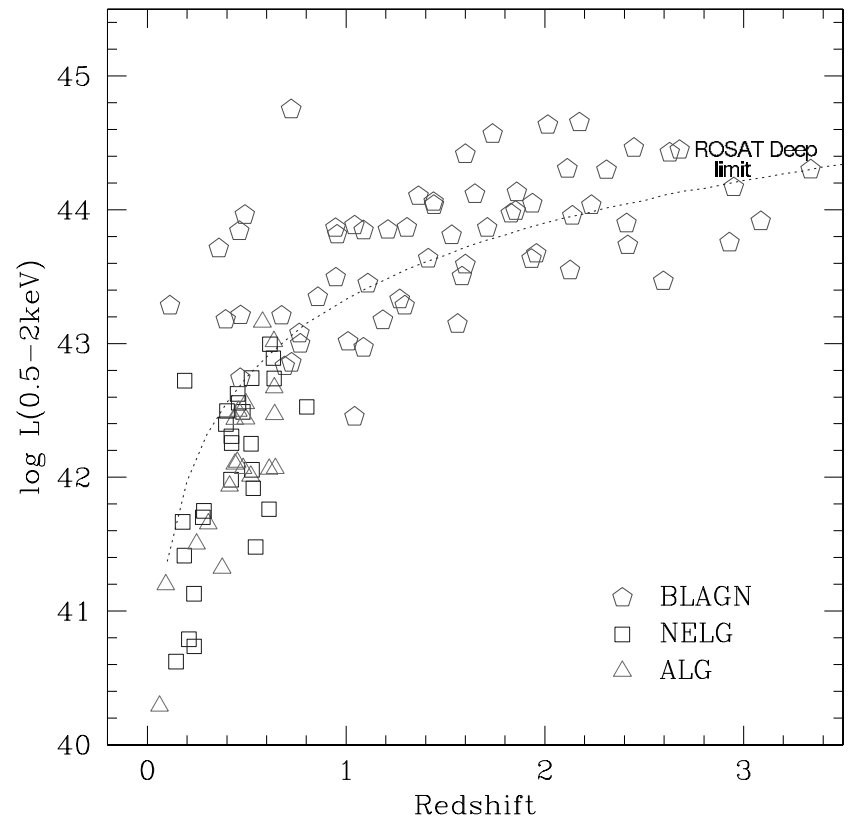

FIG. 8.-Wide coverage of the $\log L_{\mathrm{X}}$ vs. redshift plane highlights the advantages of varying depths and wide sky area of the ChaMP. BLAGNs span a higher range of $L_{\mathrm{X}}$ than galaxies but with some overlap. The dashed line shows the minimum luminosity corresponding to which the ROSAT Deep Survey was sensitive (see $\S 7.2$ ). [See the electronic edition of the Journal for a color version of this figure.]

strong dense gas outflows akin to those seen in broad absorption line (BAL) QSOs. However, the trend in these samples is primarily with $L_{\text {opt }}$ and not redshift. Another cause of the apparent trend may be a combination of wider intrinsic population dispersions in $L_{\mathrm{opt}}$ than in $L_{\mathrm{X}}$ along with superposed selection effects (Yuan et al. 1998; La Franca et al. 1995).

We detect no discernible dependence of $\alpha_{o x}$ on either redshift (Mathur, Wilkes, \& Ghosh 2002) or optical luminosity. Figure 9 shows no correlation between $\log \left(f_{\mathrm{X}} / f_{r}\right)$ and optical luminosity (which we calculate as $\nu L_{\nu}$ at $2500 \AA$, assuming optical spectral slope $\alpha=-0.5)$. Galaxies span a lower but equally wide range of $\log \left(f_{\mathrm{X}} / f_{r}\right)$ as do BLAGNs. The curve in Figure 9 traces the simulated mean quasar track with $\Delta z=0.5$ redshift bins and imposed limits of $\log f_{\mathrm{X}}=$ -15 and $r^{*}<22$. All input simulated quasars have constant $\log \left(f_{\mathrm{X}} / f_{r}\right)=-0.12\left(\alpha_{o x} \sim 1.4\right)$. The high-luminosity point has $\bar{z}=0.37$, while the faint end has $\bar{z}=4.2$. No trend is seen in BLAGNs for either data or simulation, because the accretion dominates the spectral energy distribution. By contrast, Figure 9 shows a strong correlation between $\log \left(f_{\mathrm{X}} / f_{r}\right)$ and $\mathrm{X}$-ray luminosity for galaxies. The trend is likely a combination of two effects, one intrinsic, and one extrinsic: (1) the wide range of $\log \left(f_{\mathrm{X}} / f_{r}\right)$ for galaxies may reflect the unlinking of nuclear X-ray luminosity from the optical luminosity, where the latter is strongly affected by host galaxy contributions. (2) The current optical spectroscopic magnitude limit excludes sources that are very weak in optical relative to X-ray emission (have large $f_{\mathrm{X}} / f_{r}$ ) and have small $f_{\mathrm{X}}$.

\subsection{Colors}

The optical colors of ChaMP sources depend on object type, redshift, and reddening. The SDSS provides excellent statistics on the colors of the objects that fall into their spectroscopic 

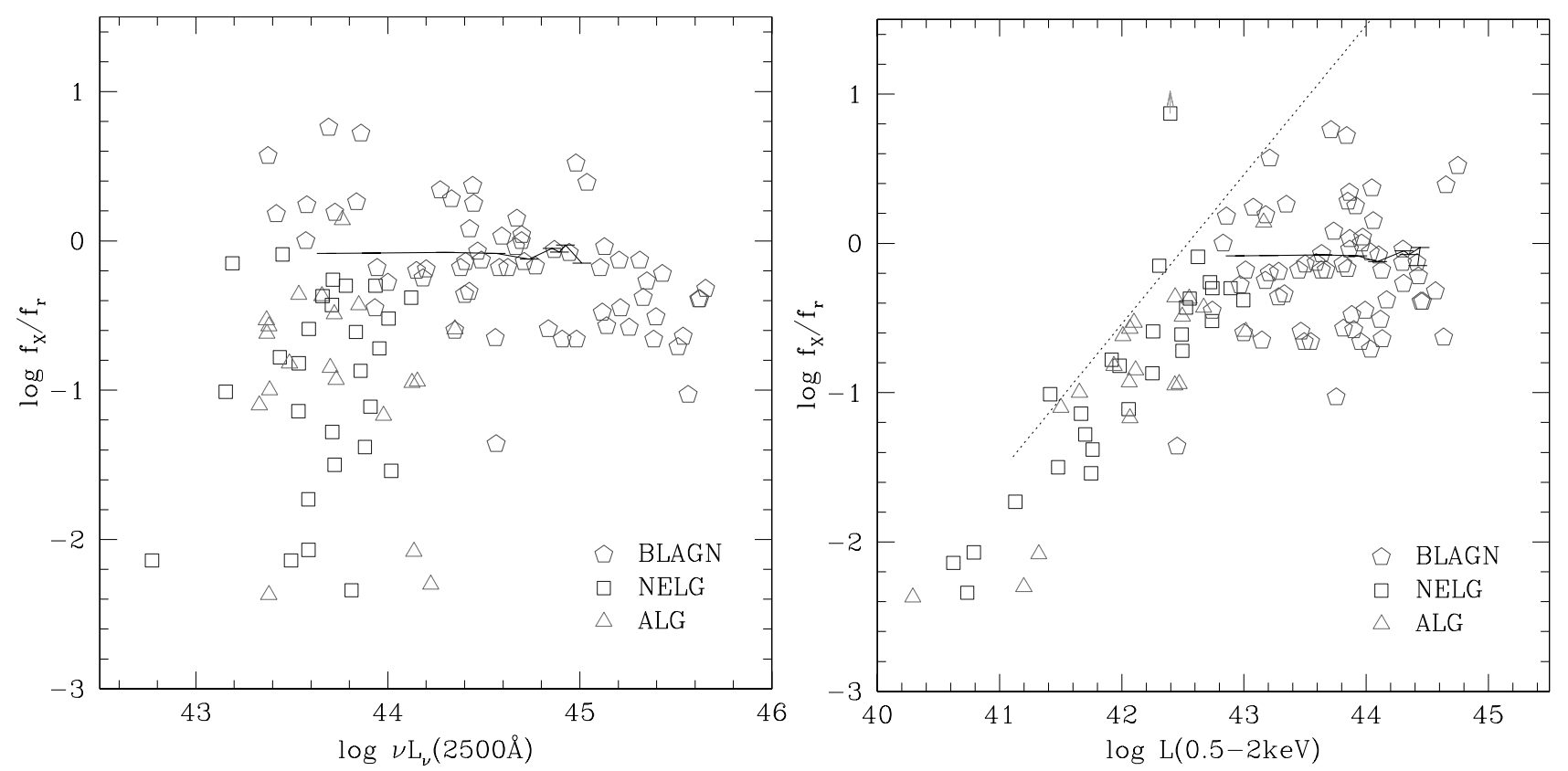

FIG. 9. - Left: $\log \left(f_{\mathrm{X}} / f_{r}\right)$ vs. $\log L_{\mathrm{opt}}$ shows that galaxies span a range of X-ray to optical flux ratio at least as wide as do BLAGNs. The curve traces the mean expected quasar track for $z=0.5$ redshift bins for the simulation described in $\S 7$ and imposed limits of $\log f_{\mathrm{X}}=-15$ and $r^{*}<22$. All input simulated quasars have constant $\log \left(f_{\mathrm{X}} / f_{r}\right)=-0.3$ (corresponding to $\left.\alpha_{o x} \sim 1.4\right)$. The high-luminosity point has $\bar{z}=0.37$, while the faint end has $\bar{z}=4.2 . \operatorname{Right}$ : $\log \left(f_{\mathrm{X}} / f_{r}\right)$ vs. $\log L_{\mathrm{X}}$ correlates strongly for galaxies but not BLAGNs. The dashed line corresponding to $r^{*}=22$ and $z=0.5$ is shown for a range of log $f_{\mathrm{X}}$ from -16 to -12 to illustrate the effect of our spectroscopic limit. The wide range of $\log \left(f_{\mathrm{X}} / f_{r}\right)$ for galaxies may reflect the unlinking of nuclear X-ray luminosity from the optical luminosity, because the latter is strongly affected by host galaxy contributions. [See the electronic edition of the Journal for a color version of this figure.]

sample. The ability to compare the photometric and color properties of X-S sources with SDSS sources is an important ChaMP feature which will be treated in upcoming papers.

Figure 10 shows the optical color distribution of ChaMP sources. The mean stellar locus in these SDSS colors is shown as two light short-dashed lines. The range of BLAGNs is similar to O-S quasars, where the expected span of $z<3$ quasars in both $\left(g^{*}-r^{*}\right)$ and $\left(r^{*}-i^{*}\right)$ is from about -0.5 to 1 (Richards et al. 2001). We plot the curve of the simulated mean quasar track for $z=0.5$ redshift bins for our simulation (with imposed flux limits of $\log f_{\mathrm{X}}=-15$ and $r^{*}=25$ ). The first point on the track to leave the stellar locus is at $\bar{z}=3.2$. Without X-ray information, normal quasars are virtually indistinguishable from stars in this color plane until higher redshifts, where quasars redden significantly in $\left(g^{*}-r^{*}\right)$, with less change in $\left(r^{*}-i^{*}\right)$. The reddest $\left(g^{*}-r^{*}\right)$ point on the track corresponds to $\bar{z}=4.6$. Galaxies are generally redder than BLAGNs. For ALGs, the $4000 \AA$ break is the strongest color determinant. The mean track of an E/S0 galaxy is shown as a red long-dashed line, using the HyperZ code of Bolzonella, Miralles, \& Pelló (2000), assuming a Bruzual-Charlot E/S0 galaxy spectrum and typical $E(B-V)=0.05$ for Galactic dereddening. The $\left(g^{*}-r^{*}\right)$ colors of $\mathrm{E}$ galaxies redden to $\left(g^{*}-r^{*}\right) \sim 2$ as the break moves redward from the $g^{\prime}$ to the $r^{\prime}$ band at $z \sim 0.4$, and the track achieves its reddest $\left(g^{*}-r^{*}\right)$ at about $z=1$ before looping back blueward to end at $z=1.2$. Several ALGs with $0.4<z<0.6$ are shown with $\left(g^{*}-r^{*}\right)$ close to 3 ; with $\log L_{\mathrm{X}} \sim 42$, these are likely to be dustreddened, low-luminosity AGNs.

The distribution of optical colors changes with X-ray flux as shown in Figure 11. In our spectroscopic sample to date, BLAGNs inhabit the full range of X-ray fluxes, but a narrow range of $\left(g^{*}-r^{*}\right)$. The brightest X-ray sources are all blue in $\left(g^{*}-r^{*}\right)$. The curve in Figure 11 traces the simulated quasar track with imposed flux limits of $\log f_{\mathrm{X}}=-15$ and $r^{*}=25$. The X-ray bright end point has $\bar{z}=0.37$, while the faint end has $\bar{z}=4.6$ Many of the faint red objects are likely to be highredshift and/or highly absorbed AGNs. X-ray faint blue objects may be the wings of the low-redshift quasar $\left(f_{\mathrm{X}} / f_{r}\right)$ distribution.

Figure 12 shows SDSS $\left(g^{\prime}-r^{\prime}\right)$ colors plotted against $\log \left(f_{\mathrm{X}} / f_{r}\right)$ in the $0.5-2 \mathrm{keV}$ band. Stars are all at $\log \left(f_{\mathrm{X}} / f_{r}\right)<-1$ and $\left(g^{*}-r^{*}\right)<1.6$. Bright BLAGNs cluster strongly at high $\log \left(f_{\mathrm{X}} / f_{r}\right)$ and blue $\left(g^{\prime}-r^{\prime}\right)$ colors. Objects in this part of the plot are largely consistent with O-S quasars and can be classified as such with high confidence even without spectroscopy; a suggested demarcation is shown as the line $\left(g^{*}-r^{*}\right)<0.3 \log \left(f_{\mathrm{X}} / f_{r}\right)+0.7$ in Figure 12. Redder objects with $\log \left(f_{\mathrm{X}} / f_{r}\right)>-1$ but $\left(g^{*}-r^{*}\right)>1$ (many undetected in our $g^{*}$-band imaging here) could be one of two types. The simulated quasar track shows that some are high-redshift $(z>4)$ quasars, but many will be obscured AGNs, as shown by the high fraction of objects without broad lines in this region.

\subsection{X-Ray Bright Galaxies with no Emission Lines}

A small number of X-ray bright, optical normal galaxies (Comastri et al. 2002) have also been found in the XMMNewton and Chandra surveys. These objects show at best weak optical emission lines. We propose three possible explanations for X-ray luminous objects showing no broad optical emission lines.

1. A "buried AGN"- a nucleus that is intrinsically similar to that of a quasar but whose optical continuum and broad emission line region are shrouded by a large obscuring column.

2. A low-luminosity AGN (LLAGN), where the host galaxy light dominates light in the spectral aperture. One such type of 


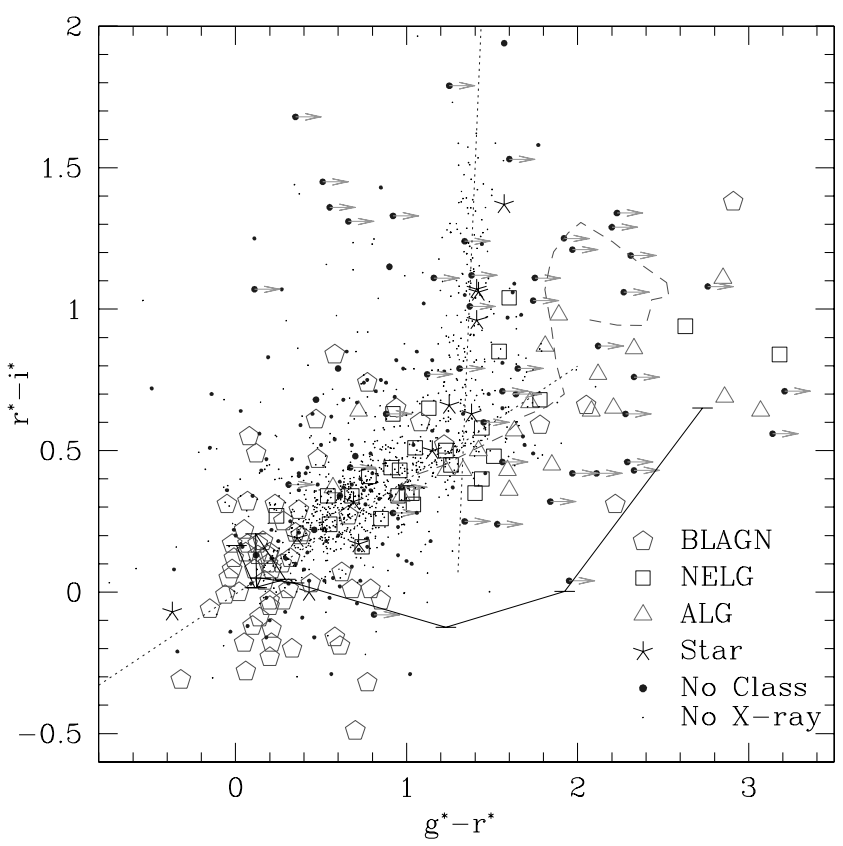

Fig. 10.- SDSS $\left(g^{*}-r^{*}\right)$ vs. $\left(r^{*}-i^{*}\right)$ color-color diagram for objects in six ChaMP fields. Optical objects with no X-ray detection are shown as small black points. The typical mean locus of SDSS objects that we use for a sanity check of our photometric calibrations is plotted as two short-dashed straight lines. The reddest BLAGN in both colors is at $z=4.93$ (Silverman et al. 2002). The solid curve traces the mean expected quasar track for $z=0.5$ redshift bins for the simulation described in $\S 7$, with imposed flux limits of $\log f_{\mathrm{X}}=-15$ and $r^{*}=25$. The first point on the track that is away from the stellar locus is at $z=3.2$, while the reddest $\left(g^{*}-r^{*}\right)$ point on the track corresponds to $\bar{z}=4.6$. Without X-ray information, normal quasars are virtually indistinguishable from stars in this color plane until $z>3.5$. The mean track of an E/S0 galaxy is shown as a red long-dashed line, using the HyperZ code of Bolzonella et al. (2000). The $\left(g^{*}-r^{*}\right)$ colors of E galaxies redden to $\left(g^{*}-r^{*}\right) \sim 2$ as the break moves redward from the $g^{\prime}$ to the $r^{\prime}$ band at $z \sim 0.4$. The loop back blueward in $\left(g^{*}-r^{*}\right)$ occurs at $z \sim 1$, where an $L^{*} \mathrm{E} / \mathrm{S} 0$ has $r^{*}=23.5$ (Blanton et al. 2001). [See the electronic edition of the Journal for a color version of this figure.]

LLAGN may host a nucleus with a massive black hole accreting at very low rates, i.e., an advection-dominated accretion flow (ADAF).

3. A BL Lac object.

\subsubsection{Buried AGNS}

The unified model explains the Seyfert classes 1 and 2 as different views of the same phenomenon (Antonucci 1993). Quasars are thought to be luminous versions of Seyfert galaxies, so this unified (orientation + obscuration) scheme should hold for quasars, i.e., they should have a similar nucleus and dusty torus system in their center. Seyfert 2 galaxies outnumber Seyfert 1 galaxies $4: 1$ in the local universe, which is consistent with the covering factor of the obscuring tori (Maiolino \& Rieke 1995), and if that ratio can be extrapolated to higher luminosities, then we should expect many type 2 quasars to be detectable in the high-redshift universe. Only a few type 2 quasars have been convincingly detected to date (Akiyama, Ueda, \& Ohta 2002; Norman et al. 2002; Stern et al. 2002; Schmidt et al. 2002), implying a strong, perhaps luminosity-dependent evolution (Franceschini et al. 2002). However, infrared surveys are currently finding bona fide type 2 quasars. Several of the IRAS hyperluminous galaxies (Beichman et al. 1986; Low et al. 1988, 1989) have been shown to be type 2 quasars (e.g., Wills et al. 1992; Hines
\& Wills 1995; Hines et al. 1995). More recently, samples selected from the 2MASS include a large number of type 2 objects with quasar-like near-IR luminosities. Fully $1 / 3$ of the spectroscopically confirmed AGNs in the 2MASS are type 2 objects (Cutri et al. 2001). Extrapolating to the entire sky, 2MASS should detect roughly 5000 such objects with $K<15$, many of which will have IR luminosities in the quasar range (Smith et al. 2002b). Near-IR surveys are less biased against absorption and less sensitive to orientation than optical surveys but will preferentially select samples with high star formation rates and copious dust.

The X-ray characteristics of 2MASS quasars suggest strong absorption $\left(N_{\mathrm{H}} \sim 10^{22} \mathrm{~cm}^{-2}\right.$; Wilkes et al. 2002) with contributions from direct and unabsorbed, scattered, and/or extended emission. The possibility remains that they are intrinsically X-ray weak. The sample of 2MASS quasars studied with Chandra by Wilkes et al. (2002) are all at $z<0.3$ (due to the 2MASS $K \sim 14.5$ limit). At higher redshifts, the reduced effective column in the X-ray bandpass should be effective in discovering type 2 quasars and help resolve the question of their intrinsic X-ray luminosities. How can and should a type 2 quasar be recognized in an X-ray survey?

It cannot be simply on the basis of X-ray hardness. High column density X-ray-absorbing gas (e.g., a warm absorber) may be interior to the putative obscuring torus, so that even normal optical type 1 (broad-line) quasars may show lowluminosity and/or high column absorption in the X-rays (however, in these cases absorption in the rest-frame UV is usually apparent; Green et al. 2001; Brandt, Laor, \& Wills 2000). Luminosity criteria may be subtle, since the claim for high- $L$ could be based on either $L_{\mathrm{opt}}$ or $L_{\mathrm{X}}$, perhaps including large absorption-corrections. We find objects in our sample that show signs of X-ray or optical absorption, or

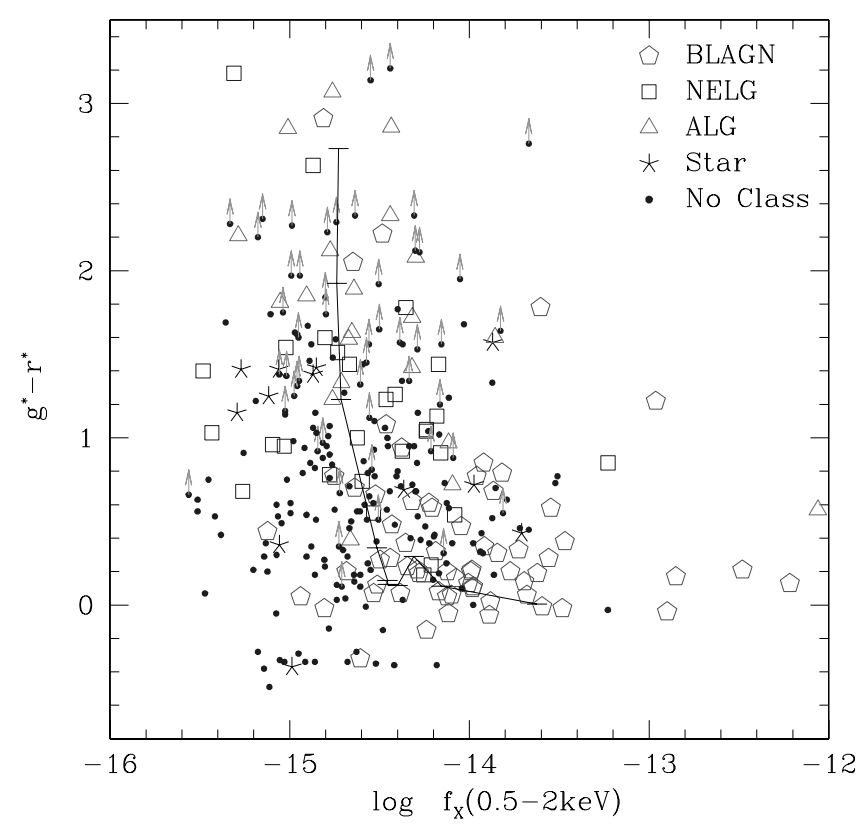

FIG. 11.- SDSS $\left(g^{\prime}-r^{\prime}\right)$ colors derived from our NOAO Mosaic imaging vs. Chandra $\log f_{\mathrm{X}}$ calculated in the $0.5-2 \mathrm{keV}$ band. Colors include limits (denoted by arrows) when the object is only detected in a single optical filter. The brightest X-ray sources are blue in $\left(g^{*}-r^{*}\right)$. The curve traces the mean expected quasar track for $z=0.5$ redshift bins for the simulation described in $\S 7$, with imposed flux limits of $\log f_{\mathrm{X}}=-15$ and $r^{*}=25$. The bright end point has $\bar{z}=0.37$, while the faint end has $\bar{z}=4.6$ [See the electronic edition of the Journal for a color version of this figure.] 


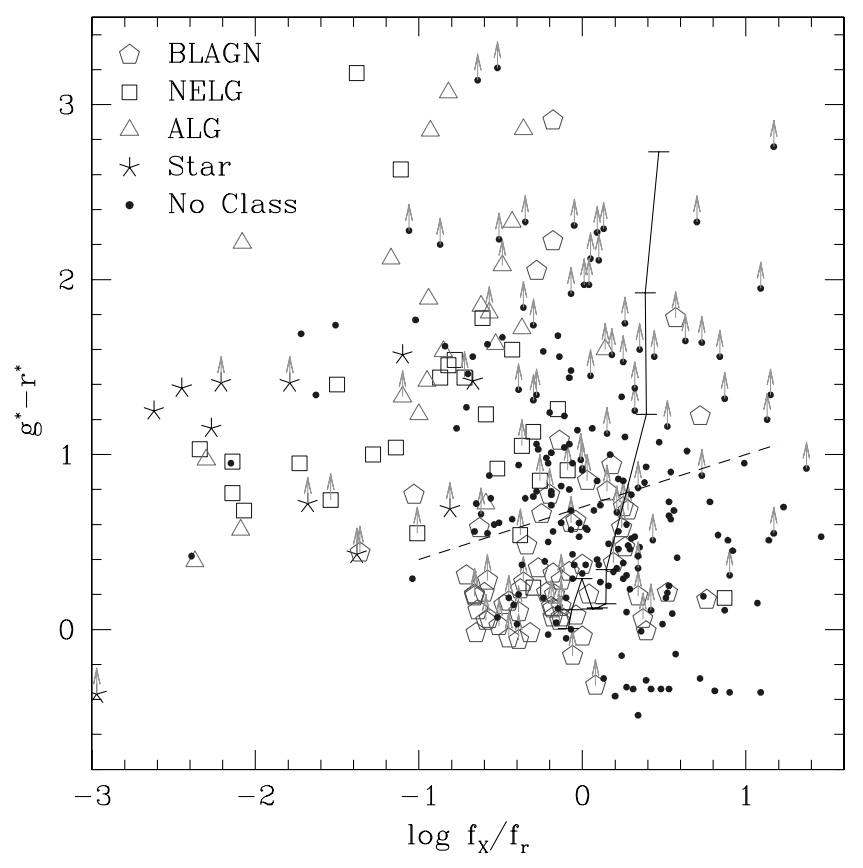

FIG. 12.- SDSS $\left(g^{\prime}-r^{\prime}\right)$ colors derived for Chandra sources from our Mosaic imaging plotted against $\log \left(f_{\mathrm{X}} / f_{r}\right)$ in the $0.5-2 \mathrm{keV}$ band. The solid curve traces the mean expected quasar track for $z=0.5$ redshift bins for the simulation described in $\S 7$, with imposed flux limits of $\log f_{\mathrm{X}}=-15$ and $r^{*}=25$. The bright end point has $\bar{z}=0.37$, while the faint end has $\bar{z}=4.6$. All input simulated quasars have constant $\log \left(f_{\mathrm{X}} / f_{r}\right)=-0.3$, so the shift toward larger $\log \left(f_{\mathrm{X}} / f_{r}\right)$ arises only from the imposed flux limits. Objects in the lower right part of the plot are largely consistent with $\mathrm{O}-\mathrm{S}$ quasars and can be classified as such with high confidence even without spectroscopy; a possible demarcation is shown as the dotted line $\left(g^{*}-r^{*}\right)<0.3 \log \left(f_{\mathrm{X}} / f_{r}\right)+0.7$. Clustered with stars at low $\log \left(f_{\mathrm{X}} / f_{r}\right)$ are galaxies that likely have little X-ray contribution from an active nucleus. [See the electronic edition of the Journal for a color version of this figure.]

both, but with no strong evidence that these properties are coupled.

If an X-S AGN is found showing no broad emission lines, oftentimes the search for broad lines may be very limited, including only what is seen on a discovery optical spectrum. A search for broad $\mathrm{H} \alpha$ is sometimes possible from the ground and may reveal instead a luminous type 1.9 quasar (Akiyama et al. 2002), but the classification is by nature somewhat arbitrary. Spectropolarimetry can reveal broad-line flux, but since those same photons must also be in the total flux spectrum, broad-line detection becomes a question of adequate S/N. Indeed, many IR-selected type 2 quasars show lower fractional polarization than type 1 quasars, presumably because of strong stellar light contributions (Smith et al. 2002b). Furthermore, there are few objects for which we can convincingly claim an optical type 2 classification without examining line ratios in detail in spectra of higher S/ N. Instead we define a "buried AGN" here as an object that has either no or only narrow emission lines in optical spectra, strong evidence for $\log N_{\mathrm{H}}>22$ in the rest frame, and $\log L_{\mathrm{X}}>43$ without absorption correction. If the signature of $\mathrm{X}$-ray absorption is clearly detected, then an absorption-corrected $\log L_{X}>44$ qualifies as a type 2 quasar candidate, with confirmation pending high $\mathrm{S} / \mathrm{N}$ follow-up spectroscopy. We find no such objects in these six fields.

\subsubsection{Low-Luminosity AGNs}

Many of these X-ray selected galaxies are so distant that their angular diameters are comparable to the slit (or fiber) widths used in ground-based spectroscopic observations. Using integrated spectra of a sample of nearby, well-studied Seyfert 2 galaxies, Moran, Filippenko, \& Chornock (2002) demonstrate that the defining spectral signatures of Seyfert 2 galaxies can be hidden by light from their host galaxies. At $z \sim 0.6$, a 1.5 slit encompasses a region about $10 \mathrm{kpc}$, which includes a substantial fraction of the host starlight emission. Some $60 \%$ of the observed objects would not be classified as Seyfert 2 galaxies on the basis of their integrated spectra, which is comparable to the fraction of Chandra sources identified as "normal galaxies" in deep surveys (Hornschemeier et al. 2001; Barger et al. 2002).

These "buried AGNs" may host low rate (advection dominated) accretion flows (ADAFs; Narayan \& Yi 1995). The $\mathrm{X}$-ray spectra produced by $\mathrm{ADAFs}$ are relatively hard, so ADAF sources may contribute a fair share of the hard $(>2 \mathrm{keV})$ background (Yi \& Boughn 1998). Half of the 2-10 keV CXRB could consist of low-luminosity $\left(L_{\mathrm{X}}<10^{41} \mathrm{ergs} \mathrm{s}^{-1}\right)$ sources if the comoving number density is $\sim 3 \times 10^{-3} \mathrm{Mpc}^{-3}$, comparable to the density of $L^{*}$ galaxies (e.g., Peebles 1993). ADAF sources should be characterized by inverted radio spectra $I_{\nu} \propto \nu^{0.4}$.

There are eight optical counterparts to X-ray sources in the current sample that have hard band ${ }^{34}$ luminosities $\log L(2-$ $8 \mathrm{keV})>43$ and no signs of broad optical emission lines in their spectra. The luminosity in this band is plotted against hardness ratio in the right panel of Figure 13. [A similar $L_{X}$ criterion in the $0.5-2 \mathrm{keV}$ band would be $\log L(0.5-2 \mathrm{keV})>$ 42.4] Half of these type 2 candidates are hard, with $\mathrm{HR}_{0}>0$. Optical spectra of this sample are shown in Figure 14. Several of these objects have spectra of absorption line galaxies with only a weak narrow [O II] emission line. Several counterparts are also classified as narrow emission line galaxies with strong lines of $\left[\mathrm{O}_{\mathrm{II}}\right],\left[\mathrm{O}_{\mathrm{III}}\right]$, and $\mathrm{H} \beta$. All these objects are at redshifts less than unity.

Newer CXRB models attempt to include the observation that most hard faint sources from Chandra and XMM-Newton surveys are objects at $z<1$, which requires a very different evolution rate for type 1 and type 2 objects. This must at least modify the meaning of unification; all type 2 AGNs are not necessarily type 1 from a different angle. The covering fraction of the putative obscuring torus may also change and probably evolves statistically with cosmic time. The relative dearth of high-redshift type 2 quasars could be explained by selection effects. As discussed in $\S 7.1$, these objects may simply fall below our optical detection limits, or at least our $r^{*}=22$ spectroscopic limit. Since these objects have low surface densities, as the ChaMP area grows, a volume-limited subsample will help define their relative space densities.

\subsubsection{BL Lac Objects?}

The first known BL Lac objects provided the three observational characteristics of the class (e.g., Stein, Odell, \& Strittmatter 1976; Angel \& Stockman 1980), repeated here as presented by Jannuzi, Smith, \& Elston (1993): “(1) they are intrinsically luminous with strong and "rapid" variability. (BL Lac objects have been observed to vary at X-ray, optical, infrared, and radio wavelengths.) (2) They have 'featureless' optical spectra. (How to define 'featureless' has been a question for debate.) (3) The electromagnetic radiation from

\footnotetext{
${ }^{34}$ While our $H$ band is nominally $2.5-8 \mathrm{keV}$, we use $2-8 \mathrm{keV}$ luminosities here to facilitate comparison to other studies.
} 

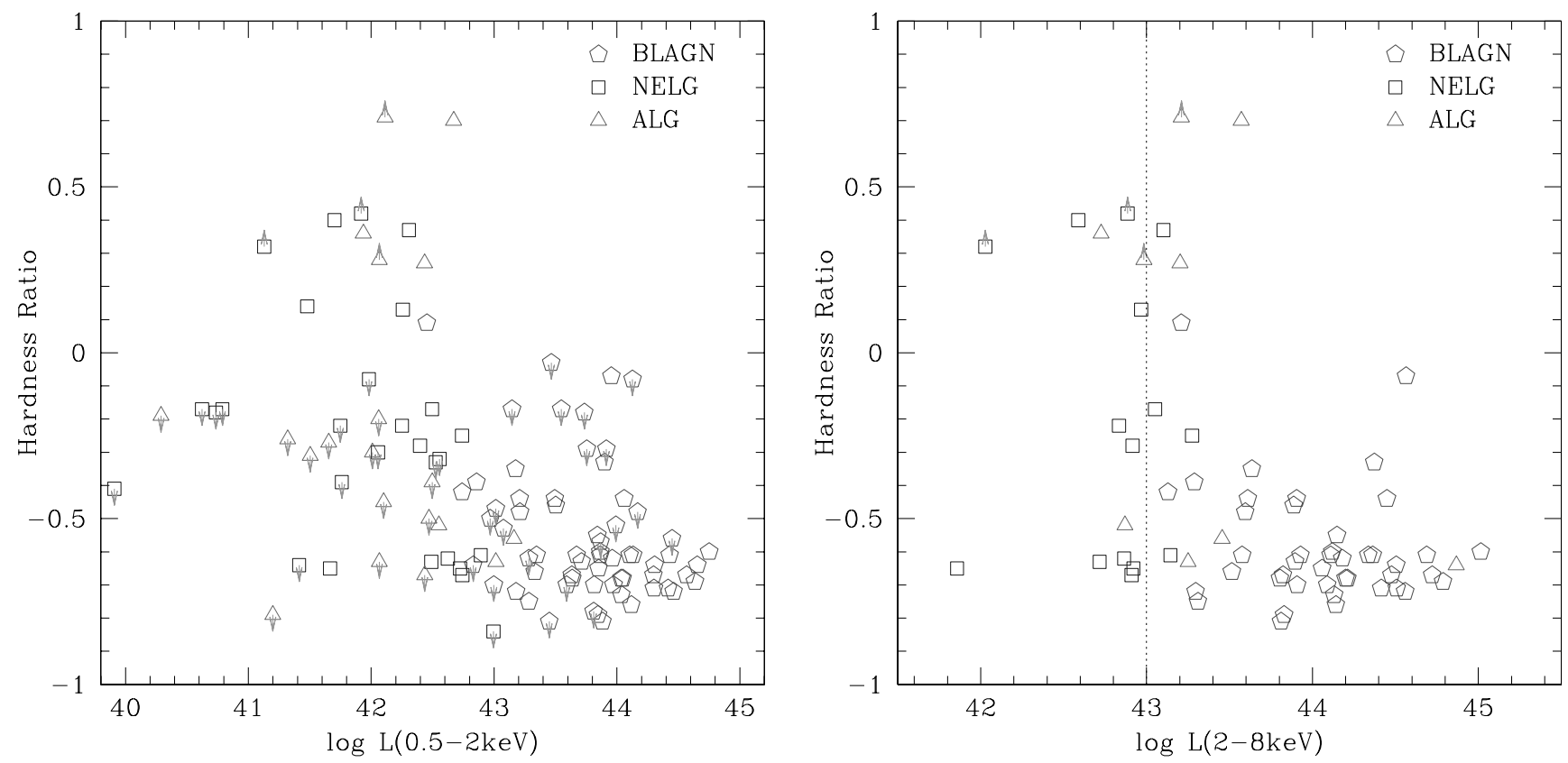

FIG. 13.-Hardness ratio vs. X-ray luminosity in two bands. Most of the luminous objects (quasars) are soft. This may arise both because they are at high redshift (where absorption causes smaller changes in hardness) and because they are intrinsically less obscured. Right: Plotting against a harder $L_{\mathrm{X}}(2-8 \mathrm{keV})$ does not significantly change the plot. A dashed line shows our criterion for objects with no broad lines to be classified as "buried AGNs"; $\log L_{\mathrm{X}}(2-8 \mathrm{keV})=43$. [See the electronic edition of the Journal for a color version of this figure.]

BL Lac objects is observed to be linearly polarized." In general, the polarization requirement has been for the observed degree of polarization to be larger than that possible from differential extinction (at most wavelengths a percentage polarization of greater than $3 \%$ ) or for the degree and/or position angle of the polarization to be variable. Together, these observationally based criteria reflect what we now know is the distinguishing physical characteristic of all BL Lac objects, the presence of a strong source of synchrotron radiation in these sources.
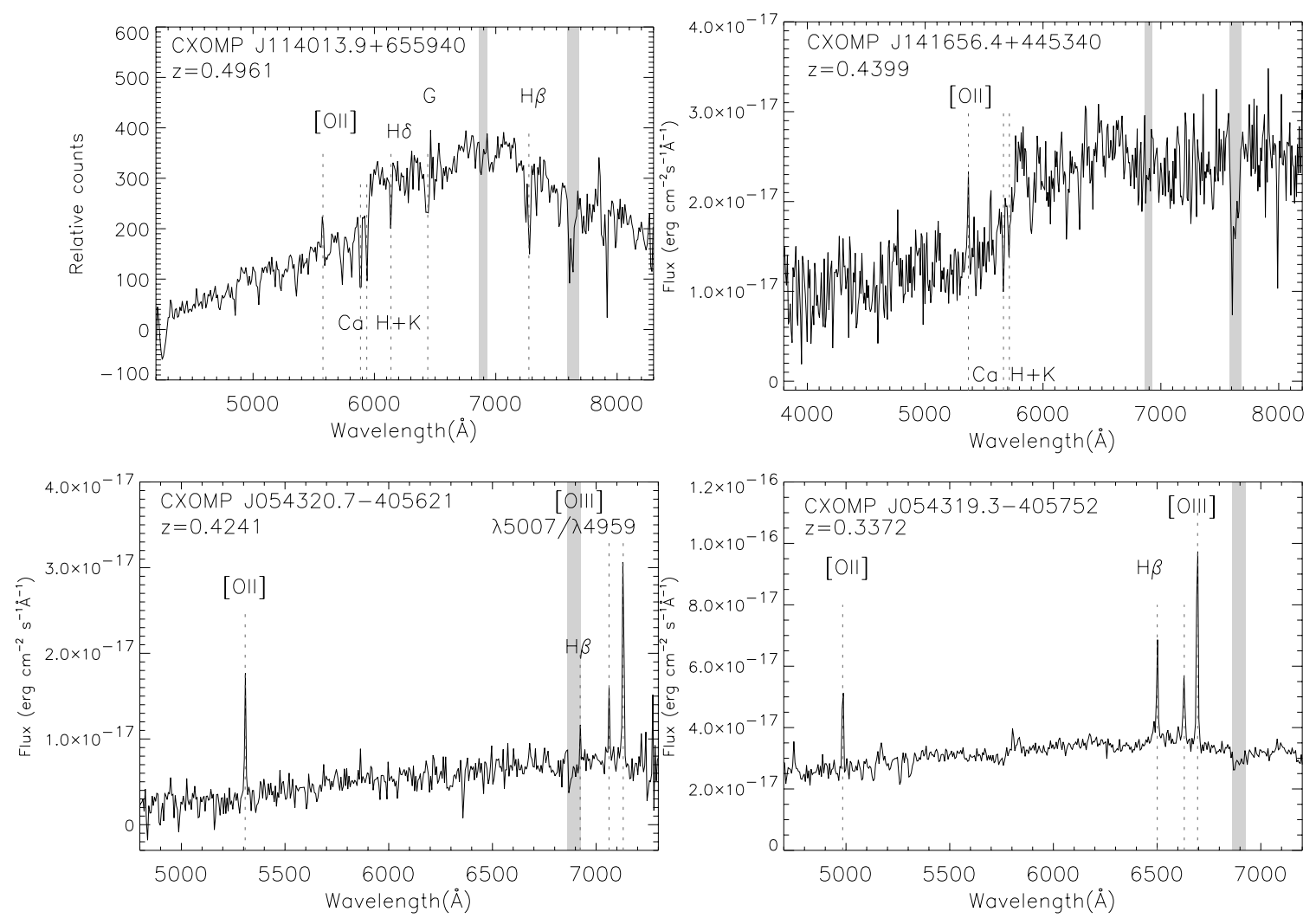

FIG. 14. - Spectra of ChaMP candidate absorbed AGNs. These optical spectra correspond to four X-ray sources with luminosities [ $\left.\log L_{\mathrm{X}}(2-8 \mathrm{keV})>43 \mathrm{ergs} \mathrm{s}{ }^{-1}\right]$, hard X-ray spectra, and no signs of broad emission lines characteristic of optically selected quasars. Gray regions mark the telluric absorption bands. 
We are interested in the identification of BL Lac objects because it is possible that with only single epoch photometry and spectroscopy (and no polarimetry data), objects that we have currently identified as buried AGNs might be BL Lac objects. Lacking strong emission lines, the optical and near-IR spectra of many BL Lac objects (particularly X-ray selected objects; see EMSS survey results of Morris et al. 1991 and Jannuzi, Elston, \& Smith 1994) can be dominated by host galaxy starlight and look very similar to what we call absorption line galaxies.

While more data on these objects would be advantageous, we have checked whether BL Lac objects might be hidden in our absorption line galaxy sample. First, we checked for known radio emission from our X-ray sample. Since all known BL Lac objects are strong radio sources (e.g., Jannuzi 1990; Stocke et al. 1990), the presence of strong emission (from a compact synchrotron core) might indicate the presence of a BL Lac. Unfortunately, no data are available of sufficient sensitivity for our ALGs. Our cross-correlation of our X-ray source list to the FIRST (White et al. 1997) and NVSS (Condon et al. 1998) radio surveys with a $5^{\prime \prime}$ search radius did yield two matches to other sources. One is a bright nearby radio-quiet Seyfert 1, and the other is likely to be a distant radio-loud quasar (see Appendix). Second, we checked the strength of the $4000 \AA$ break in our spectra of all sources we classed as ALGs or BL Lac objects (all those with the strongest emission line equivalent width $W_{\lambda}<$ $5 \AA$ ). As discussed by the EMSS team, a weak break might indicate the presence of nonthermal (featureless) emission contributing to the total emission - again a possible sign of a BL Lac being present in the source. We measured the $4000 \AA$ break contrast using the using the definition of Dressler \& Schechtman (1987)

$$
\mathrm{Br}_{4000 \AA}=\frac{f^{+}-f^{-}}{f^{+}},
$$

where $f^{+}$is the average flux in the rest wavelength range $4050-4250 \AA$ and $f^{-}$is the average flux between $3750-3950 \AA$. As long as the $\mathrm{S} / \mathrm{N}$ in this wavelength region exceeds 5 per pixel, we count break strengths less than 0.25 as BL Lac candidates pending confirmation from any of the three criteria listed above.

We found just one optical spectroscopic BL Lac candidate; CXOMP J05421.5-410206, with $r^{*}=19.6$ at $z=0.637$. While in the same range of luminosity as known BL Lac objects, it has a relatively low $\log \left(f_{\mathrm{X}} / f_{r}\right)=-0.6$. We note that for optical magnitudes $V<20$, the optical luminosity function for X-ray-selected BL Lac objects yields a surface density of about $160 \mathrm{sr}^{-1}$ or about $0.05 \mathrm{deg}^{-2}$ (Wolter et al. 1994). The optical QSO surface density is about 600 times as high at similar magnitudes (Meyer et al. 2001), so few BL Lac objects are expected overall in the ChaMP.

\subsection{Signs of Absorption?}

Figure 15 shows HR-values corrected as described in $\S 3$. Many previous studies have assumed that when total source counts exceed 30 or 50 that the HR-values are reliable without accounting for errors. In fact, the combination of source spectrum and telescope effective area may require a surprisingly large number of counts just to achieve detection in all the bands composing an HR calculation. The fraction of limits (mostly upper limits, since Chandra's $H$ band sensitivity is significantly lower) decreases below $10 \%$ only above about

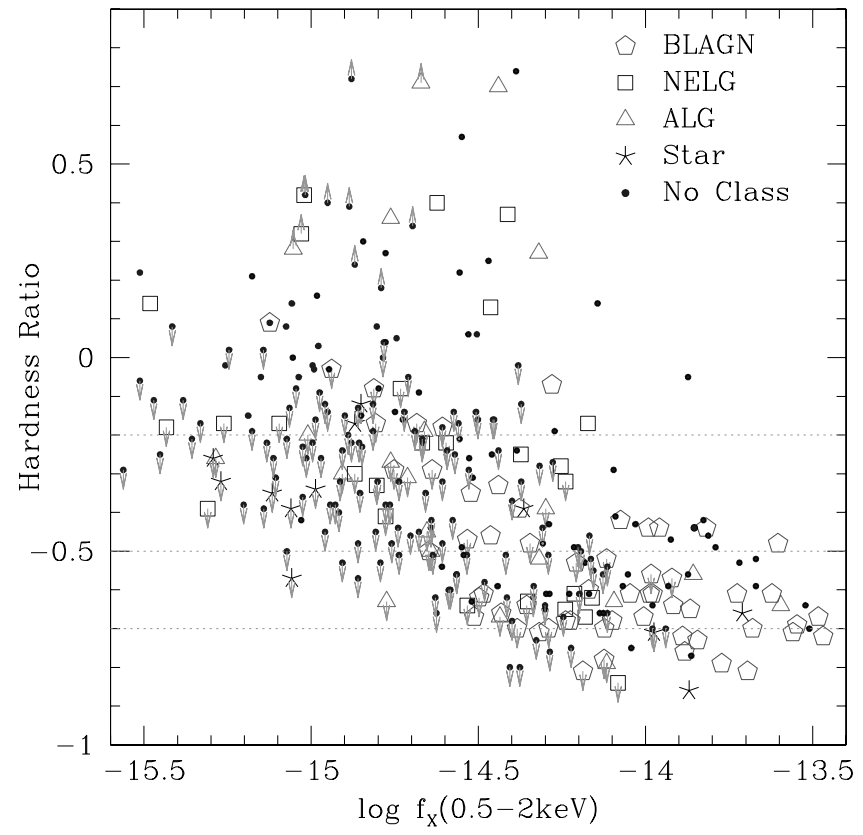

FIG. 15.-Corrected hardness ratios $\mathrm{HR}=(H-S) /(H+S)$ as a function of $\mathrm{X}$-ray flux. HR-values here are corrected to represent a source on-axis on a front-illuminated (FI) chip and to include upper or lower limits to $\mathrm{HR}_{0}$ (arrows), based on $2 \sigma$ upper limits to $H$ or $S$ counts, respectively. Horizontal dashed lines show the $\mathrm{HR}_{0}$ corresponding to a typical unabsorbed AGN (bottom; $\Gamma=$ $\left.1.9 / N_{\mathrm{H}}=0 \mathrm{~cm}^{-2}\right)$, the average hardness ratio of the total CXRB spectrum (middle; $\Gamma=1.4 / N_{\mathrm{H}}=0$ or $\Gamma=1.9 / \log N_{\mathrm{H}} \geq 21.5$ ), and a highly absorbed AGN (top; $\Gamma=1.9 / \log N_{\mathrm{H}} \geq 22$ ). Most BLAGNs likely suffer some moderate absorption, whereas a large fraction of NELG and ALG show evidence for $\log N_{\mathrm{H}} \geq 22$. The brightest eight sources between $-13.4<\log f_{\mathrm{X}}<-12$ (typically soft) are left outside the plot range for clarity. [See the electronic edition of the Journal for a color version of this figure.]

120 counts (for these observations typically at about $\log f_{\mathrm{X}}=$ -14 in Fig. 15). About half the sources have $\mathrm{HR}_{0}$ upper limits even for sources with 30 counts or more. Analysis of only those points detected in both bands would lead to an apparent hardening at low fluxes. Correctly incorporating the upper limits via survival analysis (ASURV Rev. 1.1; Isobe, Feigelson, \& Nelson 1986; LaValley, Isobe, \& Feigelson 1992), we find no significant evidence for hardening of the spectra toward lower fluxes. Excluding sources with fewer than $30(0.3-8 \mathrm{keV})$ counts, the median (mean) of $\mathrm{HR}_{0}$ for sources with fewer than 120 counts is $-0.66(-0.58 \pm 0.02)$, while for sources with more counts we find $-0.62(-0.56 \pm 0.03)$. This is not inconsistent with the conclusion in Kim et al. (2003b) that spectral hardening at faint fluxes is significant only in the softest band $(\mathrm{S} 1 ; 0.3-0.9 \mathrm{keV})$ and thus most likely due to absorption. While spectral hardening toward fainter fluxes may be real, careful accounting for errors in hardness ratios is important: spectral stacking (e.g., Brandt et al. 2001; Tozzi et al. 2001) is a powerful alternative for these analyses.

Assuming that $\Gamma \sim 1.9$ for most BLAGNs, the hardness ratios indicate that most BLAGNs likely suffer some absorption, whereas strong absorption is rare. A much larger fraction of NELG and ALG show evidence for $\log N_{\mathrm{H}} \geq 22$.

$\mathrm{X}-\mathrm{S}$ quasar samples may be less biased against absorbers (both intrinsic and line of sight) than are O-S samples. This advantage is expected to be especially important at high redshifts for two reasons. First, X-rays from high-z objects can be detected through a larger intrinsic absorbing column of gas and dust because the observed-frame X-ray bandpass 


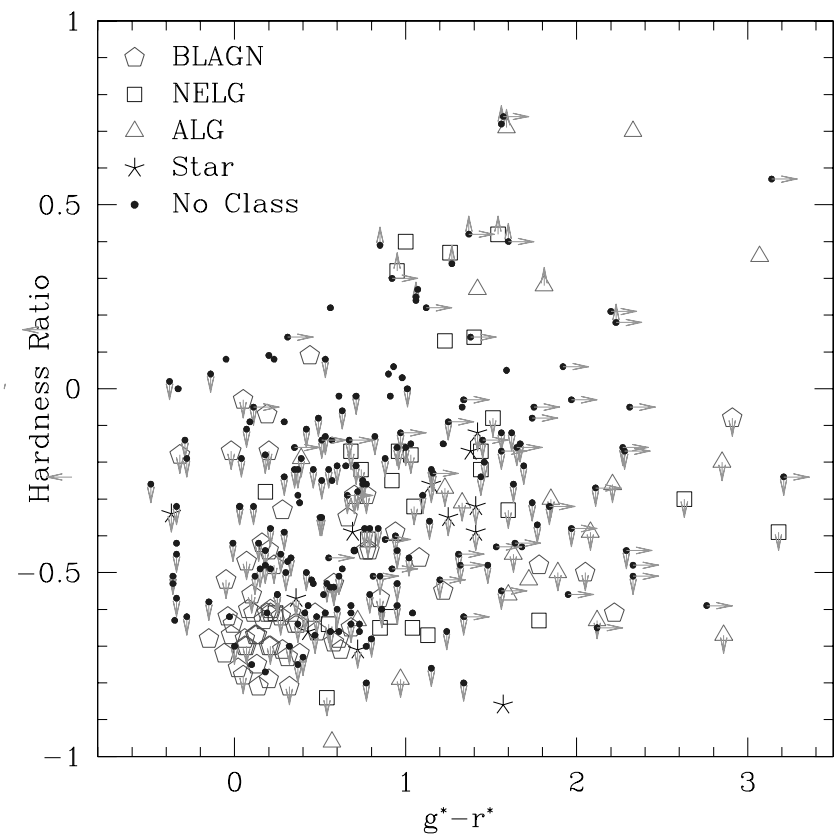

FIG. 16.- SDSS $\left(g^{\prime}-r^{\prime}\right)$ colors derived from our $4 \mathrm{~m}$ Mosaic imaging vs. corrected Chandra hardness ratio $\mathrm{HR}_{0}$. Since a range of redshifts and optical object types may pertain, $\left(g^{*}-r^{*}\right)$ is at best a crude measure of optical reddening - as is $\mathrm{HR}_{0}$ for X-ray absorbing column. Nevertheless, above $\mathrm{HR}_{0} \sim 0$ a weak correlation exists between these indicators $(P=10 \%$; see $\S 7.5)$. This suggests that at high columns, the effects of intrinsic dusty absorbers may dominate the dispersion induced by factors such as redshift, orientation, and/or reflection. [See the electronic edition of the Journal for a color version of this figure.]

corresponds to higher energy, more penetrating X-rays at the source; the observed-frame, effective absorbing column is $N_{\mathrm{H}}^{\text {eff }} \sim N_{\mathrm{H}} /(1+z)^{2.6}$ (Wilman \& Fabian 1999). Second, optical color criteria for high- $z$ candidates (e.g., blue filter dropouts) tend to select objects with strong $\operatorname{Ly} \alpha$ forest decrements.

Do red optical colors reliably predict hard X-ray spectra or vice versa? Figure 16 shows two quantities, $\mathrm{HR}_{0}$ and $\left(g^{*}-r^{*}\right)$, which may serve as crude proxies for absorption measurements. While $\left(g^{*}-r^{*}\right)$ is affected by dust via optical reddening, it is also strongly dependent on object type, host galaxy fraction, and redshift. While the hardness ratio $\mathrm{HR}_{0}$ is affected by the total X-ray absorbing column, it is also dependent on redshift since the effective column decreases as noted above. We find no overall correlation between these two absorption indicators, as is evident from Figure 16. X-ray and optical absorption are effectively decoupled for several physical reasons: (1) the gas-to-dust ratio varies, depending on densities, abundances, and ionization level (Maiolino et al. 2001); (2) physically distinct absorbing regions (Weingartner \& Murray 2002); (3) variations in geometry, reflection, and self-shielding (Elvis 2000).

We note that for the $35 \mathrm{X}$-ray hard objects $\left(\mathrm{HR}_{0}>0\right)$ suggestive of strong absorption, there is some weak evidence for a trend in Figure 16. Using ASURV (LaValley et al. 1992), we include the limits in both axes ${ }^{35}$ and find no significant correlation (probability of no correlation is 9.8\%). At high columns, other scatter-inducing factors such as redshift, orientation, and/or reflection may be overcome by the effects

\footnotetext{
35 We employ a generalization of Kendall's nonparametric rank correlation test developed by Brown et al. (1974, hereafter the BHK test). This method assumes no distribution for censored data and allows either type of censoring simultaneously in both axes.
}

of intrinsic dusty absorbers. This suggests that the correlation should be tested with a larger sample, as will become rapidly available from the ChaMP.

Hard red objects in the upper right-hand corner of the plot [e.g., $\mathrm{HR}_{0}>0$ and $\left(g^{*}-r^{*}\right)>1.5$ ] likely contain a high fraction of obscured AGNs; none with spectral classifications to date are BLAGNs. Soft red objects in the lower right [e.g., $\mathrm{HR}_{0}<0$ and $\left.\left(g^{*}-r^{*}\right)>1.5\right]$ could be high-redshift AGNs (where unabsorbed X-rays are shifted into the Chandra passband) or soft galaxies above $z \sim 0.3$. Spectra of the hard blue BLAGNs [e.g., $\mathrm{HR}_{0}>-0.2$ and $\left(g^{*}-r^{*}\right)<0.5$ ] show mostly weak narrow emission lines, and several appear to have significant blueshifted C IV absorption. These objects are similar to BALQSOs, which are only slightly reddened (Reichard et al. 2003) but known to be heavily absorbed in X-rays (Green et al. 2001).

\subsection{Clusters of Galaxies}

Clusters are important probes of cosmology, especially at high mass and redshift. In general, a surface density of more than $0.003 \mathrm{deg}^{-2}$ for clusters more massive than Coma above $z>1$ violates $\Lambda$ CDM cosmologies at $95 \%$ confidence (Evrard et al. 2002). Evolution of and correlations between X-ray temperature, luminosity, and gas mass in clusters (Vikhlinin et al. 2002) enables the strongest available constraints on hierarchical models. Chandra's high sensitivity, low background, and good spatial resolution facilitate the detection of spatially extended emission which at high Galactic latitudes corresponds to emission from groups or clusters of galaxies.

Our prototype X-ray extended source detection algorithm works by performing photometry in annuli around sources detected by wavdetect, and compares the best-fit Gaussian and beta models to the expected PSF size. All of the targeted clusters are detected. Some clusters also have pointlike emission from a cD galaxy or quasar, which can confuse the automated algorithm. These are generally noticed during our visual inspection, which also provides an opportunity to flag any X-ray source surrounded by a concentration of optical galaxies. Using this approach, we have found three serendipitous clusters in these six fields, each of which is described further below. The ChaMP makes feasible simultaneous optical and X-ray searches for clusters. Further refinement and testing of cluster selection is underway (D. A. Perley et al. 2004, in preparation) that combines automated and by-eye X-ray source extent determinations, Voronoi Tesselation and Percolation analysis on color-filtered optical images, and red sequence identification in optical color-magnitude plots.

\subsubsection{CXOMP J033912.2-352614}

During our standard visual inspection of each X-ray source, we noted extended X-ray emission centered near $03^{\mathrm{h}} 39^{\mathrm{m}} 12^{\mathrm{s}} .2$, $-35^{\circ} 26^{\prime} 15^{\prime \prime} .0$. Figure 17 shows the X-ray and optical $r^{\prime}$-band image of the region. A $\beta$ model to fit the cluster profile is unconstrained, but fixing to an average cluster value of $\beta=$ 0.6 (Jones \& Forman 1984), we derive a core-radius $r_{c}=$ $47^{\prime \prime} \pm 12^{\prime \prime}$ and 2821 counts $(0.7-2 \mathrm{keV}) .^{36}$ The $(0.5-2 \mathrm{keV})$ flux is therefore $3 \times 10^{-13} \mathrm{ergs} \mathrm{cm}^{-2} \mathrm{~s}^{-1}$. This estimate may be affected (at the $\sim 20 \%$ level) by emission from the extended halo of the central bright (10th mag) galaxy of the Fornax cluster, NGC 1399, which lies $9^{\prime}$ to the west outside the Chandra field of view (Paolillo et al. 2002).

\footnotetext{
${ }^{36}$ The cluster is only detectable to about $3 r_{c}$, within which there are 1630 counts.
} 


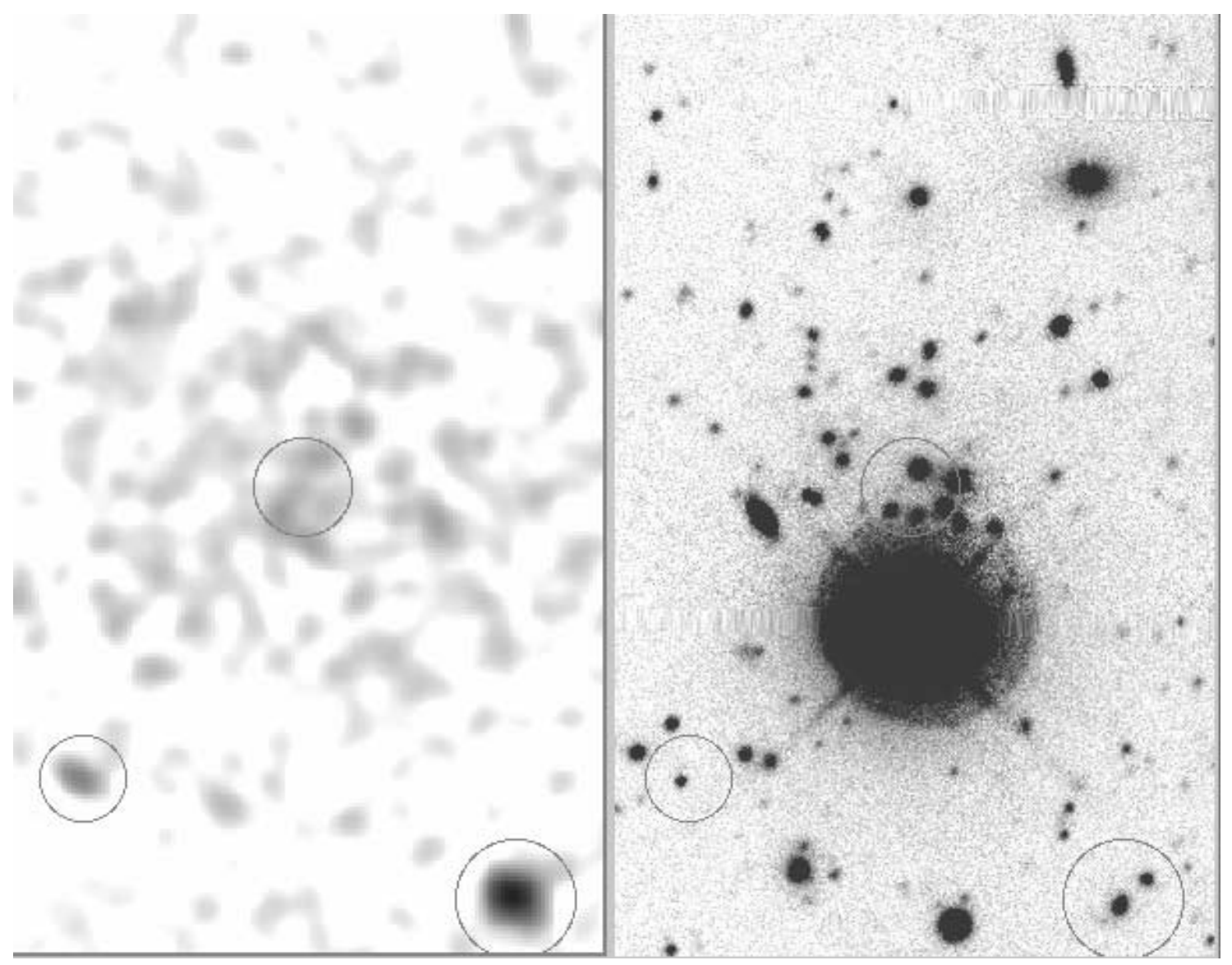

Fig. 17.-CXOMP J033912.2-352614. Left: We note extended X-ray emission centered near $\alpha=03^{\mathrm{h}} 39^{\mathrm{m}} 12^{\mathrm{s}} \cdot 2, \delta=-35^{\circ} 26^{\prime} 15^{\prime \prime}$ in this ACIS-S image from Obs. ID 624. The field is approximately $2^{\prime} \mathrm{N}-\mathrm{S}$, and 1.3 E-W. The orientation (in this and all images herein) is north up and east to the left. Circles show the positions of detected X-ray sources, with circle size corresponding to the Chandra $95 \% \mathrm{EE}$ at this off-axis angle. Right: Our CTIO $4 \mathrm{~m} /$ Mosaic imaging indeed shows a cluster of galaxies just north of a $V=12$ star. The bright star is not the X-ray source since it is 19.5 south of the X-ray centroid, and stars do not generally produce diffuse $\mathrm{X}$-ray emission. [See the electronic edition of the Journal for a color version of this figure.]

In our optical imaging, the source corresponds to what appears to be a cluster of galaxies just north of a bright ( $V=12 \mathrm{mag}$ ) star. Figure 17 shows the optical $r^{\prime}$-band image of the cluster next to the (2 pixel Gaussian) smoothed X-ray image. We are confident that the X-ray emission does not originate from the star. Stars do not produce significantly extended X-ray emission. Even if they did, the star could not reasonably be associated with the diffuse emission situated as it is at 1.7 core radii $\left(20^{\prime \prime}\right)$ from the emission centroid. As explored by D. A. Perley et al. (2004, in preparation), the $\left(g^{*}-r^{*}\right) \sim 2.5$ colors of galaxies within $18^{\prime \prime}$ of the X-ray centroid define a red sequence corresponding to cluster elliptical galaxies at $z \sim 0.4-0.5$.

\subsubsection{CXOMP J054240.9-405515}

The bright EXOSAT and ROSAT All-Sky Survey X-ray source EXO 0541.0-4056 was studied in the ASCA Medium Sensitivity Survey as 1AXG J054242-4054. Our spectrum reveals a BLAGN at $z=0.7243$, but the X-ray image clearly shows extended emission out to $\geq 40^{\prime \prime}$ from the quasar (Fig. 18). A different stretch and zoom of the same region is shown at the top of Figure 4. To better examine the cluster emission, we first created an image in the $0.7-2 \mathrm{keV}$ band only and masked out all point sources and the quasar with a circle of 4 4.5 radius. After smoothing the masked image with a $6^{\prime \prime}$ Gaussian, we determined the peak of the diffuse emission to be at $05^{\mathrm{h}} 42^{\mathrm{m}} 40^{\mathrm{s}} \cdot 4,-40^{\circ} 55^{\prime} 7^{\prime \prime} .0$, the NE of the quasar, corresponding to a projected distance of $\sim 80 \mathrm{kpc}$, assuming the quasar and cluster are at the same redshift. Since the cluster emission centroid is offset by nearly $r_{c}$ from the brightest cluster galaxy (the quasar), this cluster is not relaxed and is most likely still sloshing from a merger. The fraction of such offsets as a function of redshift is potentially a powerful probe of cluster evolution (Cohn, Bagla, \& White 2001).

Extracting a profile around the cluster centroid, we find the cluster is well fitted with a $\beta$-model of core radius $r_{c}=$ $12^{\prime \prime} \pm 2.5$, with $\beta=0.6 \pm 0.07$ and total flux of $340 \pm$ 40 counts, ${ }^{37}$ fitting down to the background intensity of 0.03 counts pixel $^{-1}$. The corresponding cluster flux of $3.6 \times$ $10^{-14} \mathrm{ergs} \mathrm{cm}^{-2} \mathrm{~s}^{-1}$ corresponds to a cluster luminosity of $10^{44} \operatorname{ergs~s}^{-1}$.

Deep imaging at fine spatial resolution also reveals evidence in the optical and near-infrared for clusters of galaxies around (radio-loud) quasars (Yee \& Ellingson 1993) at similar redshifts (Sanchez \& Gonzalez-Serrano 2002). These quasars often do not inhabit the cluster cores, which may support the interaction/merger hypotheses for their fueling, since lower velocities at large radii enhance merging efficiency (Bekki \& Noguchi 1994).

\footnotetext{
${ }^{37}$ The error bar includes the uncertainty due to the value of the $\beta$.
} 


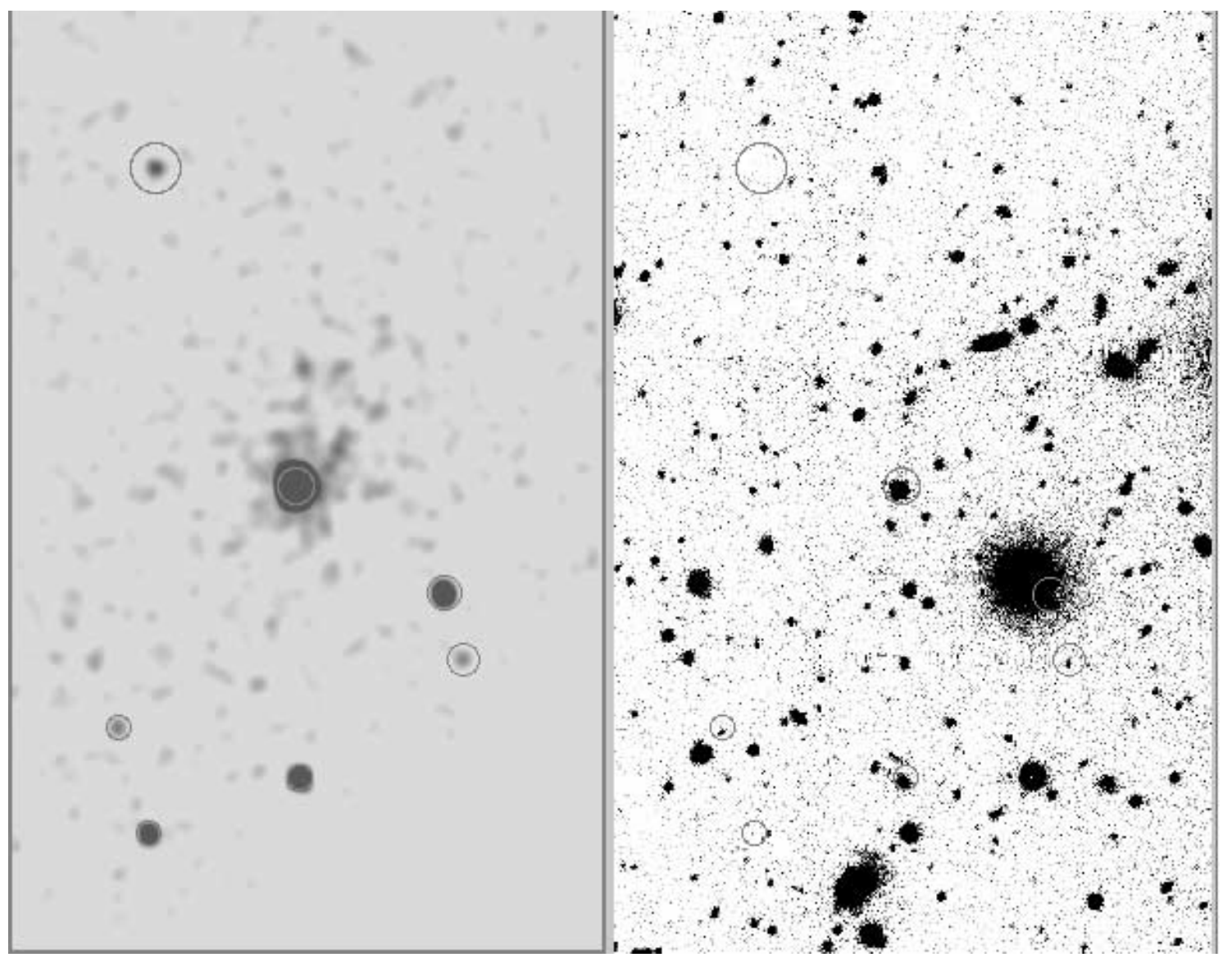

FIG. 18. - CXOMP J054240.9-405515. This source has well over 5000 counts in this ACIS-I image (left) and shows a sharp peak with significant $\left(\sim 40^{\prime \prime}\right)$ extent (see also the top panel of Fig. 4 for a close-up). Our optical spectrum of the corresponding $r^{*}=18.3$ object (right) centered in each of these images $\left(230^{\prime \prime} \mathrm{N}-\mathrm{S}\right.$, $140^{\prime \prime}$ $\mathrm{E}-\mathrm{W}$ ) reveals a $z=0.724$ BLAGN (see $\S 7.6 .2$ ). The extended emission quite likely arises in a cluster of galaxies whose members are several mag fainter than the quasar. [See the electronic edition of the Journal for a color version of this figure.]

\subsubsection{CXOMP J234815.7+005350}

The X-ray emission from this object is consistent with a point source, but the optical image looks convincingly like an intermediate redshift group or cluster of galaxies (Fig. 19). We tentatively assign a redshift of $z=0.41$ to the cluster, based on a spectrum (Magellan $6.5 \mathrm{~m}$ with LDSS2 2002 December 3) of the brightest $r^{*}=19.7$ galaxy. The source flux of $f_{\mathrm{X}}=1.0 \times$ $10^{-15} \mathrm{ergs} \mathrm{cm}^{-2} \mathrm{~s}^{-1}$ at this redshift corresponds to $\log L_{\mathrm{X}}=$ 41.84. If there is no significant contribution from cluster emission, the evidence suggests that an X-ray bright elliptical galaxy dominates the group. The object is not likely to be a BL Lac because the $\mathrm{Ca}$ II break strength is normal for an elliptical galaxy $(0.57 \pm 0.1)$. Some diffuse optical emission may be from the bright extended disk of the host galaxy ( $\sim 15 \mathrm{kpc}$ in radius), in which case a knot of optical emission near the X-ray centroid suggests the possibility of an ultraluminous X-ray source (ULX) in the host. However, this would be 30 times brighter than the brightest known ULX in the Antenna galaxies (Zezas \& Fabbiano 2002) or M82 (Kaaret et al. 2001). Also, we would expect to see strong optical emission lines from the associate star formation regions in the disk.

\section{MULTIPLE SOURCES}

Close X-ray sources $\left(<10^{\prime \prime}\right)$ with optical counterparts are good lens candidates, especially if there is also extended emission signifying a massive potential. They are also interesting for study of the effects of interactions/mergers on activity (Green et al. 2002). Sources with larger separations where one lacks an optical counterpart are good candidates for X-ray jets, whose emission barely dims at all with distance (Schwartz 2002). We have so far identified two close pairs of $\mathrm{X}$-ray sources in these six fields.

We find a new close pair of X-ray sources with optical counterparts in the field of Obs. ID 861, as shown in Figure 20. A Magellan spectrum from 2002 July 14 reveals that the brighter object CXOMP J234835.3+005832 is a $z=0.95$ BLAGN. Since the $90 \%$ encircled energy (EE) PSFs of these sources overlap, we estimated their individual counts as follows. We first measure the counts for each source in a core radius of 3 pixels. We then measure the total counts in the union of two apertures of radius of 10.4 pixels (5") encompassing both sources and apportion the total counts to each according their core counts ratio. The fainter source CXOMP J234835.5+015836 is beyond our spectroscopic survey limit but should be followed up as a lens candidate. The two objects' $f_{\mathrm{X}} / f_{r}$-values differ significantly [with $\log \left(f_{\mathrm{X}} / f_{r}\right)=0$ .72 and 0.34 for the fainter and brighter object, respectively], so we believe that they are unlikely to be lensed. However, not all lensed objects are achromatic across a wide range in frequency; microlensing, time variability, and differential absorption are possible reasons (e.g., Green et al. 2002; Morgan et al. 2001).

CXOMP J234758.0+010329 is an $r^{*}=20.1$ ALG at $z=$ 0.2481. As shown in Figure 21, CXOMP J234758.9+010343 and CXOMP J234758.0+010329 are separated by only $10^{\prime \prime}$ 


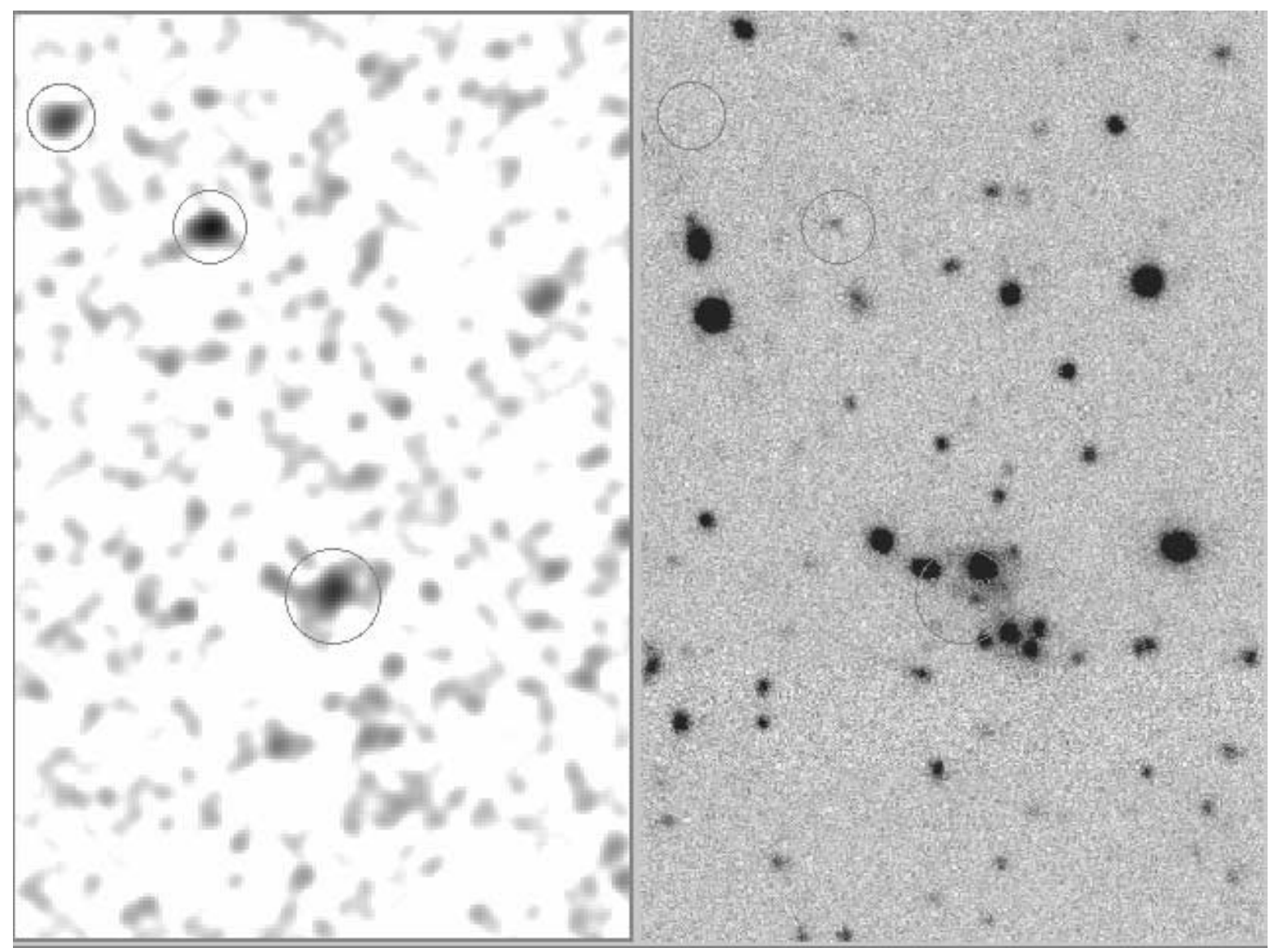

Fig. 19. - CXOMP J234815.7+005350. Left: The X-ray source has just $31 \pm 8$ counts in this ACIS-S image and morphology consistent with a point source. Circles show the $95 \%$ EE PSF size, which has radius $6^{\prime \prime}$ at this off-axis angle (4.'8). Right: The $r^{\prime}$-band image shows a group of galaxies coincident near the X-ray centroid. From our optical spectrum (Magellan $6.5 \mathrm{~m}$ with LDSS2 2002 December 3), we tentatively identify the brightest $r^{*}=19.7$ galaxy just NW of the X-ray centroid as a $z=0.4$ absorption line galaxy (see $\S 7.6 .3$ ). Both images are $240^{\prime \prime} \mathrm{N}-\mathrm{S}, 90^{\prime \prime} \mathrm{E}-\mathrm{W}$ in size. [See the electronic edition of the Journal for a color version of this figure.]

and both appear to belong to a group of galaxies. We detected no extended emission from the group.

\section{PLANS AND PROSPECTS}

Significant $(>10 \%)$ cosmic variance occurs between the $\log N-\log S$ in imaged X-ray fields (Rosati et al. 2002) at brighter fluxes $\left(\log f_{\mathrm{X}}>-14.3 \mathrm{cgs} ; 0.5-2 \mathrm{keV}\right)$, where cumulative source densities are just $130 \mathrm{deg}^{-2}(\sim 10$ sources per ACIS-I field). How strong is the X-ray cosmic variance, and is it associated with cluster-mass lenses? Using Chandra data, an X-ray point-source excess over the expectations of blank fields in the fields of several clusters has been claimed (Cappi et al. 2001; Martini et al. 2002). In the field of A2104, Martini et al. (2002) found an unexpectedly high fraction of these sources to be red cluster member galaxies both with and without optical spectroscopic signatures of AGNs. The ChaMP has a large fraction ${ }^{38}$ of target clusters in the field list, and so is well suited for testing for any such effect. Kim et al. (2003b) examine ChaMP cluster and noncluster field subsamples and compare the co-added $\log N-\log S$, finding no significant difference in point source counts.

In these six fields we have discovered three new clusters of galaxies. At least two are above $z=0.4$, while the third still

\footnotetext{
${ }^{38}$ Of the full field list, $25 \%$ have cluster targets. Of the fields with $20 \mathrm{ks}$ exposures or greater, half have cluster targets.
}

lacks a redshift. Two show X-ray extent, of which one hosts a bright quasar. Further spectroscopy is needed to verify membership of faint galaxies seen optically. Our optical imaging program will allow a new generation of studies of the clustering of X-S AGNs and of AGN-galaxy correlations. With their low surface density and high luminosities, do AGNs trace high peaks of the density field, or do they randomly sample the galaxy distribution? Is there a change in form of the AGN correlation function at small separations, implying interactions, or triggering (Efstathiou \& Rees 1988; Kochanek, Falco, \& Muñoz 1999)? What is the lifetime of activity in galactic nuclei? With the assumption that luminous quasars reside in the most massive host halos, weaker clustering implies shorter quasar lifetimes (Haiman \& Hui 2001; Martini \& Weinberg 2001).

Multiwavelength data, beyond the optical and X-ray, are also being planned in the longer term. A subset of ChaMP sources are too faint optically to be identified in our imaging program, but we have initiated ground-based near-IR imaging. This reveals many sources that are optically faint because of obscuration likely including a significant subset of the AGN population. We have also begun a program to obtain highresolution radio follow-up observations of the bright radio sources (NVSS and/or FIRST identifications) in the ChaMP sample using the VLA A array at $3.5 \mathrm{~cm}$. This not only allows us to study the radio properties of the X-S sample, but also 


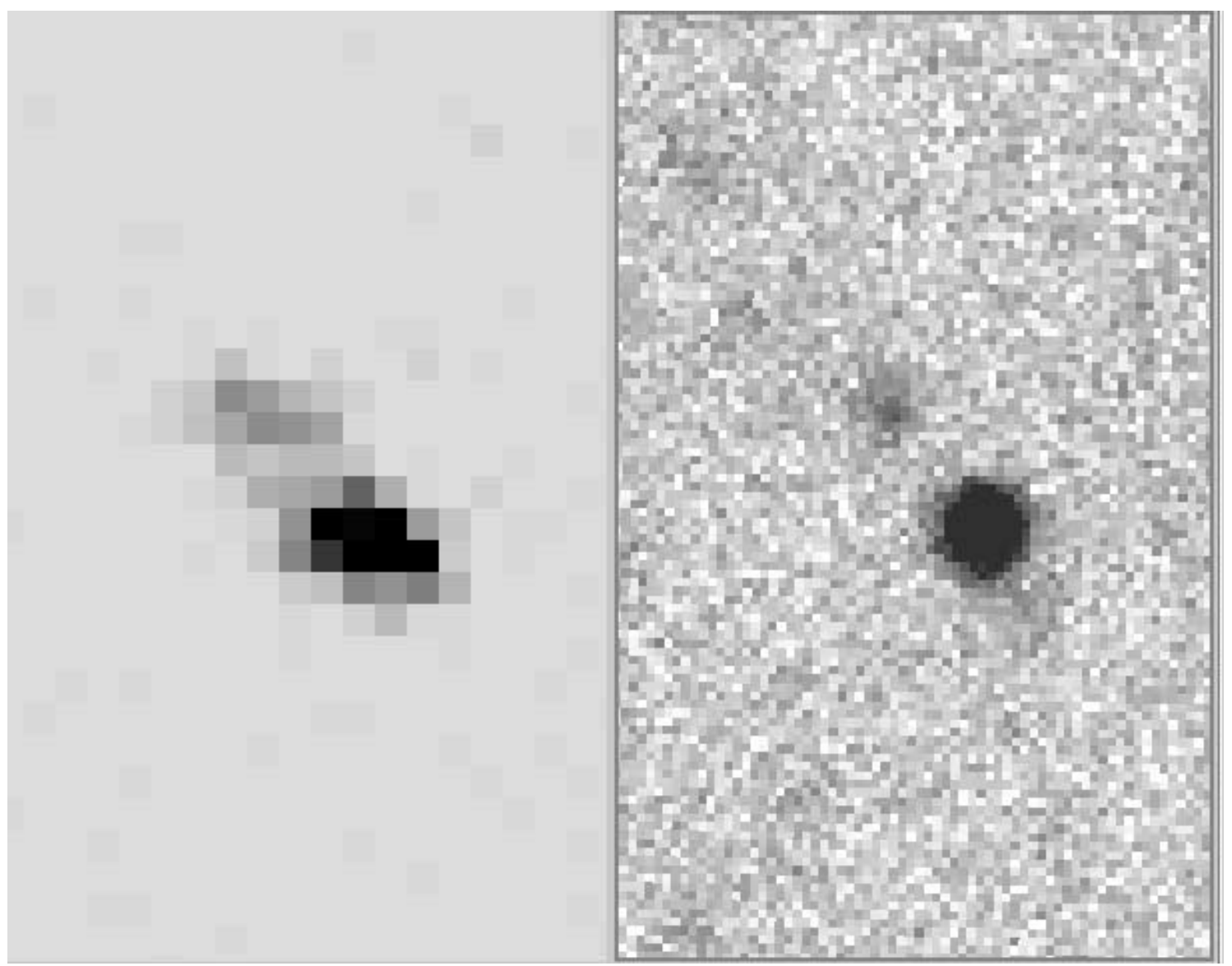

FIg. 20.-The pair of X-ray sources shown here-CXMOP J234835.3+005832 and CXOMP J234835.5+005836 - are separated by 4".9 in the ACIS-S image (left), and both have reliable optical counterparts in our Mosaic $r^{\prime}$-band image (right). The field shown is approximately $30^{\prime \prime} \mathrm{N}-\mathrm{S}$, and $18^{\prime \prime} \mathrm{E}-\mathrm{W}$. Our spectrum of the brighter more southern object shows it to be a BLAGN at $z=0.9469$. Spectroscopic follow-up of the fainter object $i^{*}=23.3$ should help determine whether the system is lensed. [See the electronic edition of the Journal for a color version of this figure.]

assists in source classification. Most of the sources observed to date are unresolved in the radio, as expected for typical broadlined AGNs. However, a significant subset have complex double/triple structure and another subset prove to be misidentifications due to the low resolution of the radio survey data. Once these observations are complete, we anticipate both extending the wavelength coverage for these bright radio sources to $20 \mathrm{~cm}$ and planning deeper observations to study the more radio-quiet part of the X-ray sample.

Beyond the mechanics of source identification and classification, a primary science goal of the ChaMP is to obtain an AGN sample which is more representative of the AGN population as a whole than previous samples, due to selection in a broad, hard X-ray band. With this sample we seek to understand the intrinsic population and study the range of spectral energy distributions and fractions of various source types in order to better constrain models for the geometry and physics of the inner regions. To some extent such studies will be facilitated by the multiwavelength data described above, but in the longer term we also expect to follow-up one or more well-defined subsets in more detail to better understand the emission mechanisms involved in the various wavebands.

\subsection{Public Data}

Quantities discussed and plotted here are available in catalog form along with image data for the six fields in this paper through the ChaMP Web site. For scientists using ChaMP catalog or image data, we appreciate an acknowledgment: "This work made use of images and/or data products provided by the Chandra Multiwavelength Project (ChaMP; Green et al. 2002), supported by NASA. Optical data for the ChaMP are obtained in part through the National Optical Astronomy Observatory (NOAO), operated by the Association of Universities for Research in Astronomy, Inc. (AURA), under cooperative agreement with the National Science Foundation."

We gratefully acknowledge support for this project under NASA under CXC archival research grant AR2-3009X. R. A. Cameron, A. Dey, P. J. Green, D.-W. Kim, D. Morris, and B. J. Wilkes also acknowledge support through NASA contract NAS8-39073 (CXC).

We are indebted to the staffs at Kitt Peak, CTIO, Las Campanas, Keck, and MMT for assistance with optical observations. Thanks to Arne Henden for accurate coordinates for standard stars, and to Warren Brown, Perry Berlind, Michael Calkins, and Susan Tokarz for providing or helping with spectroscopy on Mount Hopkins. Thanks to the referee for a thoughtful and thorough review.

Hearty thanks to the whole Chandra team for an exquisite observatory and a quality archive. We are particularly grateful to the late Leon VanSpeybroeck for allowing us early access to 


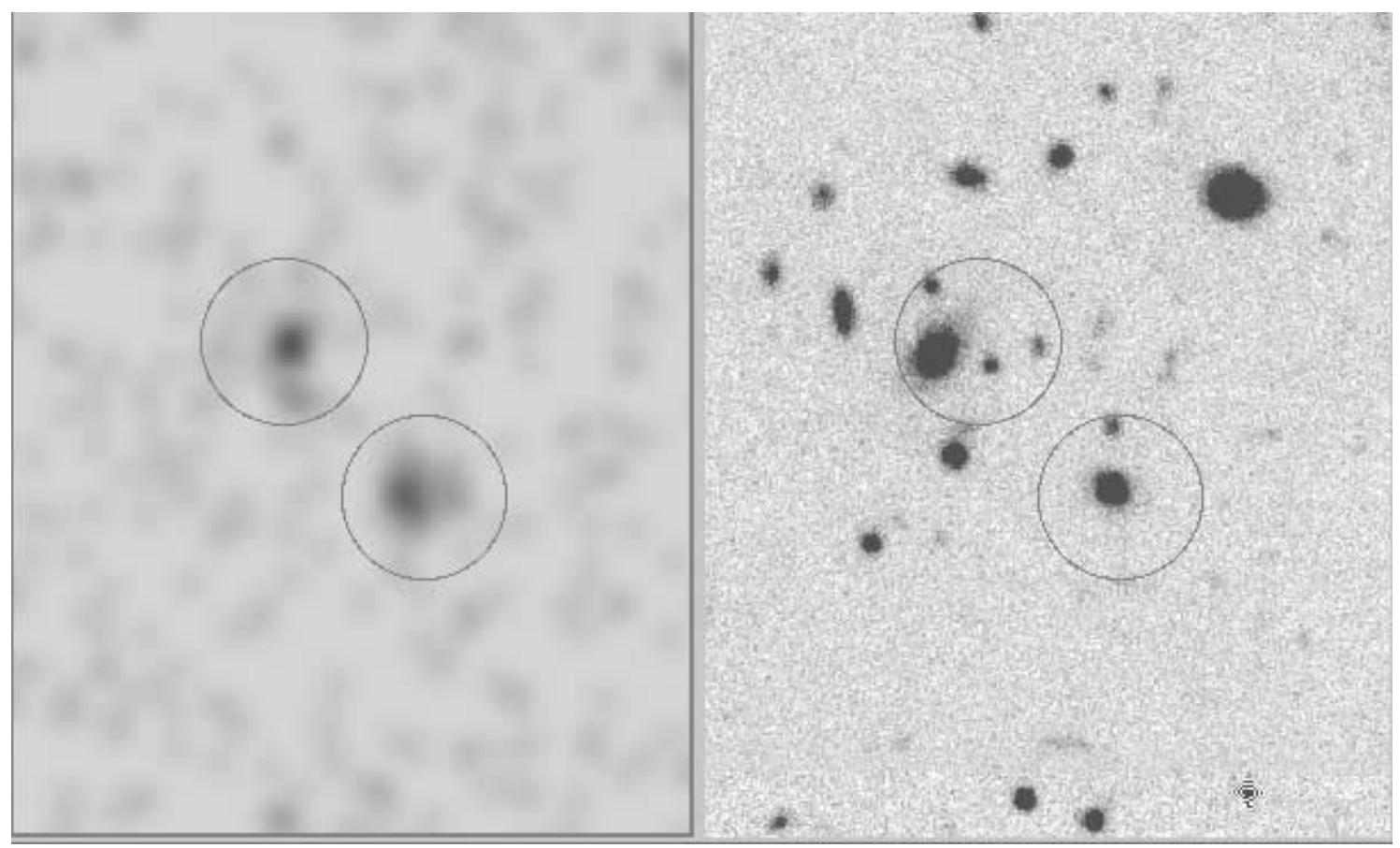

FIG. 21. - X-ray (left) and optical $r^{\prime}$-band (right) images of CXOMP J234758.9+010343 and CXOMP J234758.0+010329. Circles show the PSF size (50\% EE) at this $\left(7^{\prime}\right)$ off-axis angle. Each image is $90^{\prime \prime} \mathrm{N}-\mathrm{S}$ and $70^{\prime \prime} \mathrm{E}-\mathrm{W}$. The apparent X-ray extent of the individual sources is consistent with the Chandra PSF. The southern object CXOMP J234758.0+010329 is a $r^{*}=20.1 \mathrm{ALG}$ at $z=0.2481$. The optical counterpart centroids are separated by $19^{\prime \prime}$. While these X-ray emitting objects may belong to a group or cluster, we detect no significant extended emission. [See the electronic edition of the Journal for a color version of this figure.]

his proprietary Chandra data, and for the astonishing design quality of the Chandra, mirrors. Leon uniquely combined brilliance, integrity and humility. That inspiration and his legacy, Chandra, live on.

\section{APPENDIX}

\section{INDIVIDUAL OBJECTS IN SIX CHAMP FIELDS}

Below we note idiosyncrasies of particular fields and objects therein, if they were not discussed previously. We also mention previously known sources, based on a NED search within $12^{\prime \prime}$ of all X-ray sources. We do not tabulate or reference sources that have been detected in the growing number of all-sky or serendipitous surveys (e.g., ROSAT, SDSS, 2MASS, APM), unless a spectroscopic classification has been published.

\section{A1. MS $1137.5+6625$}

At $z=0.7824$, the target cluster MS 1137.5+6625 (PI: Leon van Speybroek) is the second most distant of the EMSS clusters (Donahue et al. 1999) and is part of a GTO program to combine X-ray and radio measurements to constrain $H_{0}$ and $q_{0}$ through the Sunyaev Zel'dovich effect. The Chandra image of the target cluster has been analyzed by Grego et al. (2003).

CXOMP J114148.0+660603. - With its $125 \mathrm{H}$ and $19 \mathrm{~S}$ band counts, this is the hardest amongst all the sources in this study $(\mathrm{HR}=0.74 \pm 0.08)$. It is relatively bright $\left(r^{*}=21.9, i^{*}=20.5\right)$, but not detected $\left(g^{*}>25.3\right)$ in the $g^{\prime}$ band.

CXOMP J114013.9+655940. - The very red color $\left[\left(g^{\prime}-r^{\prime}\right)>2.9\right]$ of this $r^{*}=21$ absorption line galaxy is unexpected for a $z=0.5$ elliptical galaxy such as this (Eisenstein et al. 2001), and even more so given that the object has a very soft X-ray spectrum $\left(\mathrm{HR}_{0}<-0.67\right)$. With $\log L_{\mathrm{X}}=42.56$, this galaxy certainly contains an AGN, but the optical light may be dominated by a reddened host galaxy.

\section{A2. V1416+4446}

V1416 +4446 is a $z=0.40$ cluster included in the same GTO program (PI: Leon van Speybroek) as MS 1137+6625.

Included within the ACIS-I field is the bright $z=0.114$ Seyfert 1 galaxy PG $1415+451$. Here there are $5067 \pm 72$ ACIS counts, corresponding to $9 \times 10^{-13} \mathrm{ergs} \mathrm{cm}^{-2} \mathrm{~s}^{-1}(0.5-2 \mathrm{keV})$. This source is also detected in the Faint Images of the Radio Sky at Twenty cm (FIRST) survey (White et al. 1997), with $1 \pm 0.14$ mJy but is nevertheless radio-quiet (Xu, Livio, \& Baum 1999).

Using a $5^{\prime \prime}$ search radius, the only other FIRST source we find in these six fields is CXOMP J141655.7+445452. With $r^{\prime}=20.94$, an $(0.5-2 \mathrm{keV})$ flux $2 \times 10^{-14} \mathrm{ergs} \mathrm{cm}^{-2} \mathrm{~s}^{-1}$ and $1.37 \pm 0.34 \mathrm{mJy}$ in the FIRST survey, it is likely to be a radio-loud quasar. 

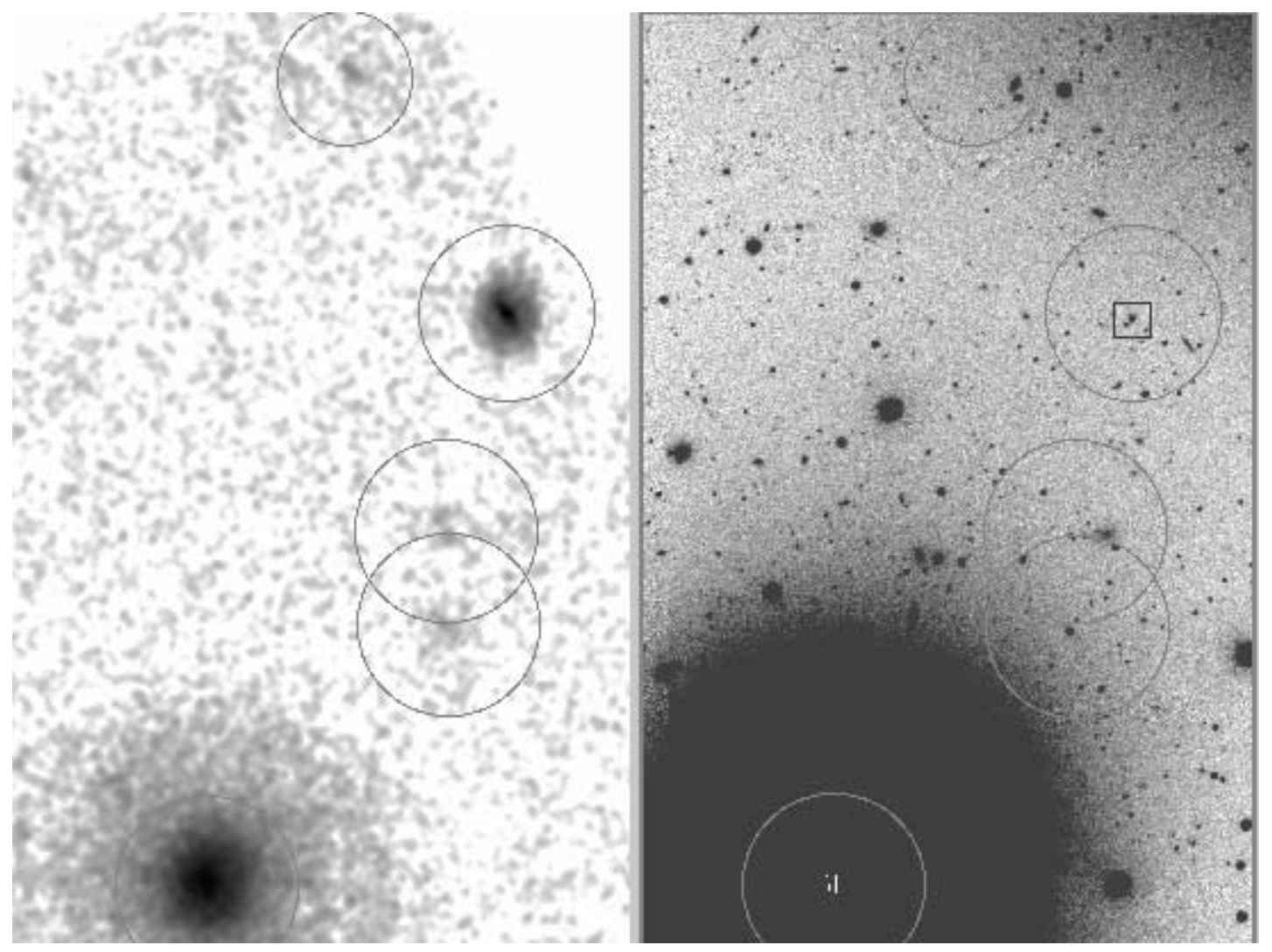

FIG. 22.-CXOMP J033841.4-353133. Smoothed ACIS-I (left) and optical $r^{\prime}$ image (right) of the field in Obs. ID 624 containing a bright background quasar (see $\S \mathrm{A} 3$ ). Each image is oriented north up, east to the left, covering 6.'7 N-S and 4.'3 E-W. The BLAGN at $z=0.3597$ is marked by a box in the optical image. Circles represent the Chandra $95 \%$ EE radius. The large (37") EE radius and apparent nonpoint morphology of the X-ray source reflect the PSF at this large (12.'6) off-axis angle. [See the electronic edition of the Journal for a color version of this figure.]

\section{A3. LP 944-20}

While the target of this observation (PI: Gibor Basri; see Rutledge et al. 2000) is a brown dwarf star, the field is near $\left(\sim 13^{\prime}\right.$ from) the center of the Fornax cluster, which includes the two bright galaxies NGC 1399 and NGC 1404. The field has been studied in the optical in detail recently by Hilker et al. (1999a, 1999b), who discovered a background galaxy cluster at $z=0.11$ behind the center of the Fornax cluster as part of the 2dF Fornax Cluster Spectroscopic Survey (FCSS; Drinkwater et al. 2000).

FCSS J033922.5-352530 (CXOMP J033922.4-352530). - This is a $B_{J}=17, r^{*}=17.56$ galaxy at $z=0.04664$.

$N G C 1404$ This is an 11th mag elliptical galaxy at $v=1947 \mathrm{~km} \mathrm{~s}^{-1}$ detected in the field with 10,710 counts. ROSAT HRI, PSPC and Chandra data on the field were studied by Paolillo et al. (2002), in which they find that the NGC 1404 halo is well represented by a single symmetric $\beta$-model and follows the stellar light profile within the inner $8 \mathrm{kpc}$. While it decreases the effective sky area available in this Obs. ID for serendipitous sources, our analyses do not include this object.

CXOMP J033841.4-353133.- Our HYDRA-CTIO spectroscopy of this bright (1710 count, $\left.r^{\prime}=20, \mathrm{HR}=-0.675\right) \mathrm{X}$-ray source shown in Figure 22 reveals a BLAGN at $z=0.3597$. Since it is in the background of the Fornax cluster, the X-ray spectrum of this quasar provides a direct measure of all the gas both neutral and ionized, along one line of sight through the cluster, the halos of both of its giant member galaxies, and the immediate environment of the quasar. We use the ACISABS model ${ }^{39}$ within CIAO Sherpa to account for the time-dependent quantum efficiency degradation of ACIS and fit an absorbed power-law model to the $\mathrm{X}$-ray spectrum of the quasar. This yields a standard AGN slope of $\Gamma=1.81 \pm 0.06$ ( $90 \%$ confidence limits), with a marginal excess absorbing column of $1.6 \pm 1.5$ over the local Galactic column (which is 1.5 , all columns in units of $10^{20} \mathrm{~cm}^{-2}$ ). Our result is consistent with those of Waugh et al. (2002), who find a considerable deficit of $\mathrm{H}$ i rich galaxies in the centre of the cluster, but our fit further suggests that little ionized gas may be present, which is worthy of further investigation.

CXOMP J033942.9-352410.-This is a previously known $r^{*}=19.0, z=1.04295$ quasar FCSS J033942.9-352410. From our HYDRA-CTIO spectra, we measure $z=1.0417$.

FCSS J033943.1-352200 (CXOMP J033943.0-352159). - This is listed as an $r=17.3$ NELG at $z=0.06208$.

FCSS J034015.4-352850 (CXOMP J034015.4-352849). - This is a $r^{*}=18.8$ quasar listed at $z=0.85504$, which assumes the bright emission line at $5225 \AA$ to be Mg II. Instead, we classify this as a $z=1.74$ BLAGN, because on the blue side of this emission line we detect the characteristic shoulder of Al III superposed on C III] $\lambda 1909$.

${ }^{39}$ See http://www.astro.psu.edu/users/chartas/xcontdir/xcont.html. 


\section{A4. Q2345+007}

The target of this Chandra image (Green et al. 2002) is a wide (7".3) separation quasar pair Q2345+007A,B ( $z=2.15)$, whose components have strikingly similar optical spectra (Steidel \& Sargent 1991). A deep search for a lensing cluster revealed nothing, and differences in the absorption X-ray hardness led the authors to posit that the system is a binary and not a lens. Evidence for or against the lens hypothesis has been sought in optical photometric variability studies, allowing a search for variable objects other than the quasars.

Three of the X-ray sources fainter than $r^{\prime}=20$ in this field are flagged as highly variable in Kochanski et al. and as such are likely to be AGNs.

CXOMP J234811.6+005700.- This $B_{J}=22$ highly variable object (G5158) has 269 counts in our ACIS image.

CXOMP J234811.9+010301 (G7676).- This object, with just 14 ACIS counts, is another such source.

CXOMP J234812.8+005750 (G5740).-This source has 203 ACIS counts.

CXOMP J234813.8+005640.- We classify this $r^{*}=20.46$ object as a $z=1.0416$ quasar $^{40}$ based on a single broad emission line at $5715 \AA$.

CXOMP J234840.1+010753.-A $z=0.71868$ quasar published in the SDSS Early Data Release (2001) as SDSS $\mathrm{J} 234840.05+01075$ quasar. We include the SDSS redshift in our tabulations.

$$
\text { A5. CL J0542.8-4100 }
$$

The intended target of this field (PI: Harald Ebeling) is a high-redshift cluster.

CXOMP J054320.2-410156.1. - The extremely X-ray bright cataclysmic variable TX Col is imaged in this field. TX Col is a highly variable member of the intermediate polar subclass of CVs. A complete study of the Chandra observation of TX Col is in preparation (A. Salinas, E. M. Schlegel, \& E. Beckerman 2004, in preparation).

CXOMP J054328.5-410119.- Our imaging reveals an $i^{*}=21$ object with complicated morphology, possibly three objects. A spectrum of the object shows an NELG at $z=0.3987$.

\section{A6. MS 2137.3-2353}

This $z=0.313$ cluster was chosen as a target (PI: Mike Wise) for study of cooling flows. None of the X-ray sources in this field yielded matches in NED.

CXOMP J213945.0-234655.- This is an X-ray selected $z=4.930$ QSO discovered by (Silverman et al. 2002) from the ChaMP.

CXOMP J214018.0-234920. - This object has an extremely blue optical spectrum, and only 1 broad line at $6735 \AA$, which we take to be rest-frame $\mathrm{Mg}$ II $\lambda 2800$ at $z=1.402 \pm 0.002$.

\footnotetext{
${ }^{40}$ Confusingly, an object at these coordinates is listed (Veron-Cetty \& Veron 2000) as a $z=2.653$ quasar with the name $2345+007 \mathrm{C}$, but these coordinates are not near the target objects that comprise $2345+007$, which in any case has $z=2.15$.
}

Akiyama, M., Ueda, Y., \& Ohta, K. 2002, ApJ, 567, 42

Akiyama, M., et al. 2000, ApJ, 532, 700

Alexander, D. M., et al. 2001, AJ, 122, 2156

Angel, J. R. P., \& Stockman, H. S. 1980, ARA\&A, 8, 321

Antonucci, R. 1993, ARA\&A, 31, 473

Barcons, X., et al. 2002, A\&A, 382, 522

Barden, S. C., Armandroff, T., Muller, G., Rudeen, A. C., Lewis, J., \& Groves, L. 1994, Proc. SPIE, 2198, 87

Barger, A. J., et al. 2001, AJ, 121, 662

. 2002, AJ, 124, 1839

Becker, R., et al. 1997, ApJ, 479, 93

Beichman, C. A., Soifer, B. T., Helou, G., Chester, T. J., Neugebauer, G., Gillett, F. C., \& Low, F. J. 1986, ApJ, 308, L1

Bekki, K., \& Noguchi, M. 1994, A\&A, 290, 7

Berk, V., et al. 2001, AJ, 122, 549

Bertin, E., \& Arnouts, S. 1996, A\&AS, 117, 393

Blain, A. W., et al. 1999, MNRAS, 309, 715

Blanton, M. R., et al. 2001, AJ, 121, 2358

Bolzonella, M., Miralles, J.-M., \& Pelló, R. 2000, A\&A, 363, 476

Boyle, B. J., et al. 1997, MNRAS, 285, 511

Brandt, W. N., Laor, A., \& Wills, B. J. 2000, ApJ, 520, 91

Brandt, W. N., et al. 2001, AJ, 122, 2810

Brown, B. W. M., Hollander, M., \& Korwar, R. M. 1974, in Reliability and Biometry, ed. F. Proschan \& R. J. Serfling (Philadelphia: SIAM), 327

Cappi, M., et al. 2001, ApJ, 548, 624

Cavaliere, A., \& Vittorini, V. 2000, ApJ, 54, 599

Chandra Proposers' Observatory Guide. 2001, Rev. 4.0 (Cambridge: Chandra X-ray Center)

\section{REFERENCES}

Chen, B., et al. 2001, ApJ, 553, 184

Comastri, A., Setti, G., Zamorani, G., \& Hasinger, G. 1995, A\&A, 296, 1 Comastri, A., et al. 2002, ApJ, 571, 771

Cohen, J. G., et al. 2000, ApJ, 538, 29

Cohn, J. D., Bagla, J. S., \& White, M. 2001, MNRAS, 325, 1053

Cole, S., \& Kaiser, N. 1989, MNRAS, 237, 1127

Condon, J. J., Cotton, W. D., Greisen, E. W., Yin, Q. F., Perley, R. A., Taylor, G. B., \& Broderick, J. J. 1998, AJ, 115, 1693

Cutri, R. M., Nelson, B. O., Kirkpatrick, J. D., Huchra, J. P., \& Smith, P. S. 2001, in ASP Conf. Ser. 232, The New Era of Wide Field Astronomy, ed. R. G. Clowes, A. J. Adamson, \& C. E. Bromage (San Francisco: ASP), 78

Dickey, J. M., \& Lockman, F. J. 1990, ARA\&A, 28, 215

Donahue, M., et al. 1999, ApJ, 527, 525

Dressler, A., \& Schechtman, S. 1987, 1987, AJ, 94, 899

Drinkwater, M. J., et al. 2000, A\&A, 355, 900

Efstathiou, G., \& Rees, M. 1988, MNRAS, 299, 229

Eisenstein, D. J., et al. 2001, AJ, 122, 2267

Elvis, M. 2000, ApJ, 545, 63

Elvis, M., Marengo, M., \& Karovska, M. 2002, ApJ, 567, L107

Elvis, M., Risaliti, G., \& Zamorani, G. 2002, ApJ, 565, L75

Elvis, M., Fiore, F., Wilkes, B., McDowell, J., \& Bechtold, J. 1994, ApJ, 422,60

1998, ApJ, 492, 91

Evrard, A. E., et al. 2002, ApJ, 573, 7

Fabian, A., \& Iwasawa, K. 1999, MNRAS, 303, L34

Fan, X. 1999, AJ, 117, 2528

Fiore, F., Elvis, M., Giommi, P., \& Padovani, P. 1998, ApJ, 492, 79 2000, NewA, 5, 143 
Forster, K., Green, P. J., Aldcroft, T. L., Vestergaard, M., Foltz, C. B., \& Hewett, P. C. 2001, ApJS, 134, 35

Franceschini, A., Braito, V., \& Fadda, D. 2002, MNRAS, 335, 51

Freeman, P. E., Kashyap, V., Rosner, R., \& Lamb, D. Q. 2002, ApJS, 138, 185

Fukugita, M., Ichikawa, T., Gunn, J. E., Doi, M., Shimasaku, K., \& Schneider, D. P. 1996, AJ, 111, 1748

Gonzalez, A. H., \& Maccarone, T. J. 2002, ApJ, 581, 155

Green, P. J., et al. 1995, ApJ, 450, 51 2001, ApJ, 558, 109 2002, ApJ, 571, 721

Gregg, M. D., Lacy, M., White, R. L., Glikman, E., Helfand, D., Becker, R. H., \& Brotherton, M. S. 2002, ApJ, 564, 133

Grego, L., et al. 2003, ApJ, submitted

Guainazzi, M., et al. 2000, A\&A, 356, 463

Haiman, Z., \& Hui, L. 2001, ApJ, 547, 27

Hardie, R. H. 1962, in Astronomical Techniques, ed. W. A. Hiltner (Chicago: Univ. Chicago Press), 178

Harris, W. E., Fitzgerald, M. P., \& Reed, B. C. 1981, PASP, 93, 507

Hasinger, G., et al. 1998, A\&A, 329, 482

2000, A\&A, 365, 45

Hilker, M., Infante, L., Viera, G., Kissler-Patig, M., \& Richtler, T. 1999a, A\&AS, 134,75

Hilker, M., Kissler-Patig, M., Richtler, T., Infante, L., \& Quintana, H. 1999b, A\&AS, 134, 59

Hines, D. C., \& Wills, B. J. 1995, ApJ, 448, L69

Hines, D. C., Schmidt, P. S., Cutri, R. M., \& Low, F. J. 1995, ApJ, 450, L1 2001, ApJ, 554, 742

Howell, S. B. 1989, PASP, 101, 616

Isobe, T., Feigelson, E. D., \& Nelson, P. I. 1986, ApJ, 306, 490

Jacoby, G. H., Hunter, D. A., \& Christian, C. A. 1984, ApJS, 56, 257

Jannuzi, B. T. 1990, Ph.D. thesis, Univ. Arizona

Jannuzi, B. T., \& Dey, A. 1999, in ASP Conf. Ser. 191, Photometric

Redshifts and the Detection of High Redshift Galaxies, ed. R.

Weymann, L. Storrie-Lombardi, M. Sawicki, \& R. Brunner (San

Francisco: ASP), 111

Jannuzi, B. T., Elston, R., \& Smith, P. S. 1994, ApJ, 428, 130

Jannuzi, B. T., Smith, P. S., \& Elston, R. 1993, ApJS, 85, 265

Jones, C., \& Forman, W. 1984, ApJ, 276, 38

Kaaret, P., et al. 2001, MNRAS, 321, L29

Kim, D.-W., et al. 2003a, ApJS, submitted 2003b, ApJ, submitted

Kochanek, C., Falco, \& Muñoz, J. A. 1999, ApJ, 521, 17

Kron, R. G. 1980, ApJS, 43, 305

Kümmel, M. W., \& Wagner, S. J. 2001, A\&A, 370, 384

Kuraszkiewicz, J., et al. 2002, ApJ, 581, 77

La Franca, F., Franceschini, A., Cristiani, S., \& Vio, R. 1995, A\&A, 299, 19

Landolt, A. U. 1992, AJ, 104, 340

Lenz, D. D., Newberg, H. J., Rosner, R., Richards, G. T., \& Stoughton, C. 1998, ApJS, 119, 121

LaValley, M., Isobe, T., \& Feigelson, E. D. 1992, in ASP Conf. Ser. 25, Astronomical Data Analysis Software and Systems I, ed. D. M. Worrall, C. Biemesderfer, \& J. Barnes (San Francisco: ASP), 245

Lehmann, I., et al. 2001, A\&A, 371, 833

Low, F. J., Cutri, R. M., Huchra, J. P., \& Kleinmann, S. G. 1988, ApJ, 327, L41

Low, F. J., Cutri, R. M., Kleinmann, S. G., \& Huchra, J. P. 1989, ApJ, $340, \mathrm{~L} 1$

Mainieri, V., et al. 2002, A\&A, 393, 425

Maiolino, R., Marconi, A., Salvati, M., Risaliti, G., Severgnini, P., Oliva, E., La Franca, F., \& Vanzi, L. 2001, A\&A, 365, 28

Maiolino, R., \& Rieke, G. H. 1995, ApJ, 454, 95

Manners, J. C., et al. 2003, MNRAS, 343, 293

Martini, P., Kelson, D. D., Mulchaey, J. S., \& Trager, S. C. 2002, ApJ, 576, L109

Martini, P., \& Weinberg, D. H. 2001, ApJ, 547, 12

Masci, F. J., Drinkwater, M. J., \& Webster, R. L. 1999, ApJ, 510, 703

Mathur, S., Wilkes, B. J., \& Ghosh, H. 2002, ApJ, 570, L5

Merritt, D., \& Ferrarese, L. 2001, MNRAS, 320, 30

Meyer, M. J., Drinkwater, M. J., Phillipps, S., \& Couch, W. J. 2001, MNRAS, 324, 343

Miyaji et al. 2000, A\&A, 353, 25

Moran, E. C., Filippenko, A. V., \& Chornock, R. 2002, ApJ, 579, L71

Moretti, A., Campana, S., Lazzati, D., \& Tagliaferri, G. 2003, ApJ, 588, 696

Morgan, N. D., Chartas, G., Malm, M., Bautz, M. W., Burud, I., Hjorth, J., Jones, S. E., \& Schechter, P. L. 2001, ApJ, 555, 1
Morris, S. L., Stocke, J. T., Gioia, I. M., Schild, R. E., Wolter, A., Maccacaro, T., \& della Ceca, R. 1991, ApJ, 380, 49

Muller, G. P., Reed, R., Armandroff, T., Boroson, T. A., \& Jacoby, G. H. 1998, Proc. SPIE, 3355, 577

Mukai, K. 1993, HEASARC Newslett., 3, 21

Narayan, R., \& Yi, I. 1995, ApJ, 444, 231

Newberg, H. J. 1999, ApJS, 123, 377

Nonino, M., et al. 1999, A\&AS, 137, 51

Norman, C., et al. 2002, ApJ, 571, 218

Oke, J. B., et al. 1995, PASP, 107, 375

Osmer, P. S. 1982, ApJ, 253, 28

Osterbrock, D. E. 1993, ApJ, 404, 551

Page, M. J., et al. 1996, MNRAS, 281, 579

Paolillo, M., Fabbiano, G., Peres, G., \& Kim, D.-W. 2002, ApJ, 565, 883

Peebles, P. J. E. 1993, Principles of Physical Cosmology (Princeton: Princeton Univ. Press)

Reeves, J. N., et al. 1997, MNRAS, 292, 468

Reichard, T. A., et al. 2003, AJ, 125, 1711

Richards, G. T., et al. 2001, AJ, 122, 1151

Rosati, P., et al. 2002, ApJ, 566, 667

Rutledge, R. E., Basri, G., Martín, E. L., \& Bildsten, L. 2000, ApJ, 538, L141

Sanchez, S. F., \& Gonzalez-Serrano, J. I. 2002, A\&A, 396, 773

Schlegel, D. J., Finkbeiner, D. P., \& Davis, M. 1998, AJ, 500, 523

Schmidt, M., Schneider, D. P., \& Gunn, J. E. 1995, AJ, 110, 68

Schmidt, G., Smith, P., Foltz, C. B., \& Hines, D. C. 2002, ApJ, 578, L99

Schneider, D. P., et al. 2002, AJ, 123, 567

Schwartz, D. A. 2002, ApJ, 571, 71

Shaver, P. A., Wall, J. V., Kellermann, K. I., Jackson, C. A., \& Hawkins, M. R. S. 1996, Nature, 384, 439

Silverman, J., et al. 2002, ApJ, 569, L1

Smith, J. A., et al. 2002a, AJ, 123, 2121

Smith, P. S., Schmidt, G. D., Hines, D. C., Cutri, R. M., \& Nelson, B. O. 2002b, ApJ, 569, 23

Steidel, C. C., \& Sargent, W. L. W. 1991, AJ, 102, 1610

Stein, W. A., Odell, S. L., \& Strittmatter, P. A. 1976, ARA\&A, 14, 173

Stern, D., et al. 2002, ApJ, 568, 71

Stocke, J. T., Morris, S. L., Gioia, I., Maccacaro, T., Schild, R. E., \& Wolter, A. 1990, ApJ, 348, 141

Stocke, J. T., et al. 1991, ApJS, 76, 813

Tozzi, P., et al. 2001, ApJ, 2001, ApJ, 562, 42

Valdes, F. G. 2002, in Automated Data Analysis in Astronomy, ed. R. Gupta, H. P. Singh, \& C. A. L. Bailer-Jones (New Delhi: Narosa Publishing House), 309

Veron-Cetty, M.-P., \& Veron, P. 2000, VizieR On-line Data Catalog: $\mathrm{VII} / 215$

Vester, J., et al. 2001, A\&A, 366, 7

Vignali, C., Brandt, W. N., Fan, X., Gunn, J. E., Kaspi, S., Schneider, D. P., \& Strauss, M. A. 2001, AJ, 122, 2143

Vignali, C., et al. 2003, AJ, 125, 2876

Vikhlinin, A., VanSpeybroeck, L., Markevitch, M., Forman, W. R., \& Grego, L. 2002, ApJ, 578, L107

Warren, S. J., Hewett, P. C., \& Osmer, P. S. 1994, ApJ, 421, 412

Watson, M. G. 2001, A\&A, 365, 51

Waugh, M., et al. 2002, MNRAS, 337, 641

Weingartner, J. C., \& Murray, N. 2002, ApJ, 580, 88

White, R. L., Becker, R. H., Helfand, D. J., \& Gregg, M. D. 1997, ApJ, 475,479

Wilkes, B. J., Tananbaum, H., Worrall, D. M., Avni, Y., Oey, M. S., \& Flanagan, J. 1994, ApJS, 92, 53

Wilkes, B. J., et al. 2002, ApJ, 564, L65

Wills, B. J., Wills, D., Evans, N. J., Natta, A., Thompson, K. L., Breger, M., \& Sitko, M. L. 1992, ApJ, 400, 96

Wilman, R. J., \& Fabian, A. C. 1999, ApJ, 522, 157

Wilman, R. J., Fabian, A. C., \& Nulsen, P. E. J. 2000, MNRAS, 319, 583

Wolter, A., Caccianiga, A., della Ceca, R., \& Maccacaro, T. 1994, ApJ, 433,29

Xu, C., Livio, M., \& Baum, S. 1999, AJ, 118, 1169

Yasuda, N., et al. 2001, AJ, 122, 1104

Yee, H. K. C., \& Ellingson, E. 1993, ApJ, 411, 43

Yi, I., \& Boughn, S. P. 1998, ApJ, 499, 198

Yu, Q., \& Tremaine, S. 2002, MNRAS, 335, 965

Yuan, W., Brinkmann, W., Siebert, J., \& Voges, W. 1998, A\&A, 330, 108

Yuan, W., Matsuoka, M., Wang, T., Ueno, S., Kubo, H., \& Mihara, T. 2000, ApJ, 545, 625

Zezas, A., \& Fabbiano, G. 2002, ApJ, 577, 726 